\title{
LIMIT THEOREMS FOR MULTI-TYPE GENERAL BRANCHING PROCESSES WITH POPULATION DEPENDENCE
}

\author{
JIE YEN FAN, KAIS HAMZA, PETER JAGERS, AND FIMA C. KLEBANER
}

\begin{abstract}
A general multi-type population model is considered, where individuals live and reproduce according to their age and type, but also under the influence of the size and composition of the entire population. We describe the dynamics of the population density as a measure-valued process and obtain its asymptotics, as the population grows with the environmental carrying capacity. "Density" in this paper generally refers to the population size as compared to the carrying capacity. Thus, a deterministic approximation is given, in the form of a Law of Large Numbers, as well as a Central Limit Theorem. Migration can also be incorporated. This general framework is then adapted to model sexual reproduction, with a special section on serial monogamic mating systems.
\end{abstract}

\section{INTRODUCTION}

Classical stochastic population dynamics (branching processes of various generality) assume independently acting individuals, usually in stable circumstances, whereas deterministic approaches, while paying attention to the feedback loop between a population and its environment, tend to sweep dependence among individuals under the carpet. In a series of papers, branching processes with population size dependence have been studied, first in the discrete time Galton-Watson case [23, 24, more recently also for single-type general processes [7, 10, 11, 18, 19], where [7] can be viewed as a single-type companion paper to the present. A first approach to general multi-type processes, comprising sexual reproduction, was made in 20.

In this paper, we introduce a general model, where an individual is characterised by its age and type. Reproduction and death may depend on these as well as on the environment (the size and composition of the whole population). This is achieved by describing the population as a measure-valued process, as done in 7 . The dependence on the composition of the population could be, for instance, on the size of subpopulations of different types or ages. The resulting general model has a high level of flexibility and can be adapted to the dynamics of quite diverse biological populations.

In an ecological community, species interact; reproduction and death depend on the composition of the community. This complex structure can be approached in our setup. In particular, in predator-prey models, species can be viewed as types,

2010 Mathematics Subject Classification. 60J80,60F05,92D25.

Key words and phrases. Age and type structure dependent population processes, size dependent reproduction, carrying capacity, law of large numbers, central limit theorem, diffusion approximation.

Supported by the Australian Research Council Grants DP120102728, DP150103588, and the Knut and Alice Wallenberg Foundation. 
and in food webs (e.g. 31]), many complex interactions occur, where age, sex, and other types are important.

Another example is the modelling of cell proliferation and differentiation, which has been studied by multi-type branching processes (e.g. [13, 30]). One particular phenomenon is that of the formation of oligodendrocytes: progenitor cells dividing into new progenitor cells, differentiating into oligodendrocytes, or dying. Here a detailed description of the cell population and its evolution can be given, for high cell densities.

In the second part (Section 3) of this paper, we give a special case of sexual reproduction, where couple formation is considered, in a (serial) monogamy system. In nature such systems can have quite varying forms from season-long to life-long couples, persisting until the death of one of the partners. The approach can be adapted to different strategies of couple formation.

Proofs are to be found in Appendices - Appendix $\mathrm{A}$ for Section 2 and Appendix B for Section 3 .

Naively speaking, our object of study is the process describing how the number of individuals $S_{t}^{K}(B \times A)$, with ages in an interval $A$ and types in a set $B$, evolves as time $t \geqslant 0$ passes, for large $K>0$. Here, $K$ historically is the habitat carrying capacity, more generally interpretable as a system size parameter. Mathematically, thus, we consider measure-valued processes $\left(S_{t}^{K}\right)_{t \geqslant 0}$, as $K \rightarrow \infty$. The process is supposed to start from a measure $S_{0}^{K}$, the total mass of which is of the order $K$, and the evolvement of $S_{t}^{K}$ is governed by birth and death intensities that depend on individual age and type, population structure, and $K$.

\section{Multi-type population structure dynamics}

2.1. The model set-up. The population model is defined as in [7, but with some additional characteristics. It builds upon the Ulam-Harris family space, with type inherited from mother to child, as described in [17. An individual $x=x_{1} x_{2} \cdots x_{n}$ is thought of as the $x_{n}$ th child of $\cdots$ of the $x_{2}$ th child of the $x_{1}$ th ancestor and

$$
I:=\bigcup_{n=1}^{\infty} \mathbb{N}^{n}
$$

denotes the set of possible individuals. In particular, $x j$ denotes the $j$ th child of individual $x$.

Each individual $x$ born into the population is characterised by its type, $\kappa_{x}$, and birth time, $\tau_{x}$, which is recursively defined as the birth time of its mother plus her age at bearing the individual, cf. 12, 16, 17. Individuals age at rate 1 until death. Let $\lambda_{x}$ denote the lifespan of $x$ and write $\sigma_{x}=\tau_{x}+\lambda_{x}$ for the death time. Then at each time $\tau_{x} \leqslant t<\tau_{x}+\lambda_{x}$ the individual will be in state $s_{x}(t)=\left(\kappa_{x}, t-\tau_{x}\right) \in \mathbb{K} \times \mathbb{A}=: \mathbb{S}$. We write $\mathbb{K}$ for the set of possible types, assumed to be finite; and $\mathbb{A}$ for the set of possible ages, assumed to be a bounded interval $[0, \omega]$ with $\omega<\infty$ denoting the maximal age (following classical demographic notation), which in the present case will be defined in Section 2.2

The composition of the population at time $t$ can be represented by the measure

$$
S_{t}(d i, d v)=\sum_{x \in I} \mathbf{1}_{\tau_{x} \leqslant t<\sigma_{x}} \delta_{\left(\kappa_{x}, t-\tau_{x}\right)}(d i, d v),
$$

where $\delta_{s}$ denotes the Dirac measure at $s$, assigning unit mass to $s$. Thus, $S_{t}$ is a measure with unit mass at the state of each individual that is alive at time $t$. (In 
this and later we allow ourselves to suppress writing the dependence upon carrying capacity $K$ and often also upon time $t$.) We shall denote the set of finite nonnegative measures on $\mathbb{S}$, with its weak topology, by $\mathcal{M}(\mathbb{S})$, or $\mathcal{M}$ for short. Thus $S_{t} \in \mathcal{M}$ for each $t$. Here $\mathbb{S}$ has the product topology of discrete and Euclidean topology, of course.

The initial population $S_{0}$ is assumed to be finite and deterministic. Suppose that bearing and death times are stochastically given by type, age and population dependent rates. An individual of state $s$ in a population composition $S$ gives birth at rate $b_{S}(s)$ until it dies, with the death intensity being $h_{S}(s)$. At each birth event, a number of offspring of type $i \in \mathbb{K}$ is generated, with the distribution of a random variable $\check{\xi}_{S}^{i}(s)$. Similarly, offspring may be born at the death of the mother (splitting) with the distribution of the random variable $\widehat{\xi}_{S}^{i}(s)$. We reiterate that the suffix $S$ represents the population composition, which could include population size and other aspects of the population structure. Alternatively, the reproduction process could have been given as an integer valued random measure on $\mathbb{S}$, like in [17, disintegrated into a stream of events and a random mass at each event. The variables $\check{\xi}_{S}^{i}(s)$ then have the Palm distribution, given a birth event at $s \in \mathbb{S}$, 15, 22. Those pertaining to the same mother but at different $s$ are also assumed independent.

Remark 1. Indeed, the birth rate pertaining to each individual $x$ with $\tau_{x}<\infty$ gives rise to a point process of bearings by $x$ at ages $0<\alpha_{x}^{1}<\alpha_{x}^{2}<\ldots<\lambda_{x}$. (For simplicity, we disregard the possibility of immediate bearing at birth, [16]. Births at death (splitting) are handled separately in this paper.) The random variable $\check{\xi}_{S_{\tau_{x}+\alpha_{x}^{j}}^{x, i}}\left(\kappa_{x}, \alpha_{x}^{j}\right)$ that stands for the number of type $i$ children born by $x$ at time $\tau_{x}+\alpha_{x}^{j}$, then follows the distribution of $\check{\xi}_{S}^{i}(s)$, with $S=S_{\tau_{x}+\alpha_{x}^{j}}$ and $s=\left(\kappa_{x}, \alpha_{x}^{j}\right)$. A corresponding remark is valid for the number of children generated at death. We shall not enter into the awkward details of this but refer to the construction in [17. At the individual level, the construction there is, however, more general than the present not only through a richer type space but also since earlier reproduction history may influence the propensity to give birth.

The population model can be described in either of two ways, through the generator of the process (as in [18 and [20]), or through the evolution equation of the process (as in [7]), see Section A.1. Before giving the dynamic equation, we clarify the concepts of differentiation and integration on $\mathbb{S}$. Derivatives of a function on $\mathbb{S}$ refer to the derivatives with respect to the second, continuous variable, i.e. age. In particular, for $f: \mathbb{S} \rightarrow \mathbb{R}$ and $s=(i, v)$, we write $f^{(j)}$ to mean

$$
f^{(j)}(s)=f^{(j)}(i, v)=\partial_{v}^{j} f(i, v),
$$

where $\partial_{v}^{j}$ denotes the $j$ th derivative with respect to the variable $v$. We also use $f^{\prime}$ for $f^{(1)}$. If $\mu$ is a Borel (positive or signed) measure on $\mathbb{S}$, then for $f: \mathbb{S} \rightarrow \mathbb{R}$, we write

$$
(f, \mu)=\int_{\mathbb{S}} f(s) \mu(d s)=\int_{\mathbb{K} \times \mathbb{A}} f(i, v) \mu(d i \times d v)=\sum_{i \in \mathbb{K}} \int_{\mathbb{A}} f(i, v) \mu(\{i\} \times d v) .
$$

For non-negative integers $j$, we write $C^{j}(\mathbb{S})$ for the space of functions on $\mathbb{S}$ with continuous derivatives (with respect to the age variable) up to order $j$. Since we 
only consider a bounded domain $\mathbb{S}$, functions in $C^{j}(\mathbb{S})$ are bounded and so are the $j$ derivatives. We can define the norm

$$
\|f\|_{C^{j}(\mathbb{S})}=\max _{0 \leqslant \iota \leqslant j} \sup _{s \in \mathbb{S}}\left|f^{(\iota)}(s)\right|=\max _{0 \leqslant \iota \leqslant j} \sup _{i \in \mathbb{K}, v \in \mathbb{A}}\left|f^{(\iota)}(i, v)\right|,
$$

and will use $\|\cdot\|_{C^{0}}$ and $\|\cdot\|_{\infty}$ interchangeably.

We write $\breve{m}_{S}^{i}(s)=\mathbb{E}\left[\breve{\xi}_{S}^{i}(s) \mid S\right], i \in \mathbb{K}$, for the expectation of the number of type $i$ progeny at the birth event in question, given population size and composition, and the individual's state at that time. Note that the expectation is that of a random variable having the specified conditional distribution and that the conditional covariances of the number of children born by the same mother at two different bearing events vanish. Similarly, we write $\breve{\gamma}_{S}^{i_{1} i_{2}}(s)=\mathbb{E}\left[\check{\xi}_{S}^{i_{1}}(s) \check{\xi}_{S}^{i_{2}}(s) \mid S\right], i_{1}, i_{2} \in \mathbb{K}$ and define $\hat{m}_{S}^{i}(s)$ and $\hat{\gamma}_{S}^{i_{1} i_{2}}(s)$ for $\hat{\xi}$ in a similar vein. Then, the mean intensity of births of an individual of state $s$ at time $t$ is $\sum_{i} \breve{m}_{S_{t}}^{i}(s) b_{S_{t}}(s)+\sum_{i} \hat{m}_{S_{t}}^{i}(s) h_{S_{t}}(s)$.

For $f \in C^{1}(\mathbb{S})$,

$$
\left(f, S_{t}\right)=\left(f, S_{0}\right)+\int_{0}^{t}\left(L_{S_{u}} f, S_{u}\right) d u+M_{t}^{f}
$$

where

$$
L_{S} f=f^{\prime}-h_{S} f+\sum_{i \in \mathbb{K}} f(i, 0)\left(b_{S} \check{m}_{S}^{i}+h_{S} \hat{m}_{S}^{i}\right)
$$

and $M_{t}^{f}$ is a locally square integrable martingale with predictable quadratic variation

$$
\begin{aligned}
\left\langle M^{f}\right\rangle_{t}=\int_{0}^{t}\left(\sum_{i_{1} \in \mathbb{K}} \sum_{i_{2} \in \mathbb{K}} f\left(i_{1}, 0\right) f\left(i_{2}, 0\right)\right. & \left(b_{S_{u}} \breve{\gamma}_{S_{u}}^{i_{1} i_{2}}+h_{S_{u}} \hat{\gamma}_{S_{u}}^{i_{1} i_{2}}\right) \\
& \left.+h_{S_{u}} f^{2}-2 \sum_{i \in \mathbb{K}} f(i, 0) h_{S_{u}} \hat{m}_{S_{u}}^{i} f, S_{u}\right) d u .
\end{aligned}
$$

For simplicity of notation, we shall write

$$
n^{i}=b \breve{m}^{i}+h \widehat{m}^{i} \quad \text { and } \quad w^{i_{1} i_{2}}=b \breve{\gamma}^{i_{1} i_{2}}+h \widehat{\gamma}^{i_{1} i_{2}}
$$

from here onwards.

Differential equation for specific characteristic of the population can be obtained from an appropriate test function $f$ (and $F$ ). For example, if $f=1$, the result is the population size; taking $f(i, v)=v$, yields the sum of the ages of the population. It is also possible to count individuals of a certain type; for instance, taking $f(i, v)=$ $\mathbf{1}_{i=1}$, we have the size of the subpopulation of type 1 , and similarly the average age of individuals of a type can be obtained.

Remark 2. The above results can be extended to test functions on $\mathbb{S} \times \mathbb{T}$, where $\mathbb{T}$ is a time interval $[0, T]$. Consider test functions $f(s, t) \equiv f(i, v, t)$ on $\mathbb{S} \times \mathbb{T}$. We shall write $f_{t}(s)$ to mean $f(s, t)$ and use the two notations interchangeably. For such $f, \partial_{1} f$ refers to the derivative with respect to the variable $s$ (or $v$ in this case), and $\partial_{1} f$ refers to the derivative with respect to $t$. For $f \in C^{1,1}(\mathbb{S} \times \mathbb{T})$,

$$
\left(f_{t}, S_{t}\right)=\left(f_{0}, S_{0}\right)+\int_{0}^{t}\left(\partial_{1} f_{u}+\partial_{2} f_{u}-f_{u} h_{S_{u}}+\sum_{i \in \mathbb{K}} f_{u}(i, 0) n_{S_{u}}^{i}, S_{u}\right) d u+M_{t}^{f},
$$


where $M_{t}^{f}$ is a martingale with the predictable quadratic variation

$$
\begin{aligned}
\left\langle M^{f}\right\rangle_{t}=\int_{0}^{t}\left(\sum_{i_{1} \in \mathbb{K}} \sum_{i_{2} \in \mathbb{K}} f_{u}\left(i_{1}, 0\right) f_{u}\left(i_{2}, 0\right) w_{S_{u}}^{i_{1} i_{2}}+h_{S_{u}} f_{u}^{2}\right. & \\
& \left.-2 \sum_{i \in \mathbb{K}} f_{u}(i, 0) h_{S_{u}} \hat{m}_{S_{u}}^{i} f_{u}, S_{u}\right) d u .
\end{aligned}
$$

Remark 3. Using the same argument as in [7, Section 5.2], we can show the existence of a measure $M_{t}$ such that $\left(f, M_{t}\right)=M_{t}^{f}$, and define the martingale $\int_{0}^{t}\left(f_{u}, d M_{u}\right)$ for $f \in C^{0}(\mathbb{S} \times \mathbb{T})$. (See Section A.1.3.)

2.1.1. Applications: sexual reproduction. A simple application of the stochastic process introduced above, where individual life can be influenced by many factors like individual type and age, population size, and population structure, is to model sexual reproduction. Let $\mathbb{K}=\{1,2\} \equiv\left\{\right.$ o, $\left.\sigma^{\prime}\right\}$ with type 1 (denoted as o) representing females (the reproducing type) and type 2 (denoted as $\sigma^{\prime}$ ), representing males, so that $b_{S}(q, v) \geqslant 0$ and $b_{S}\left(\sigma^{*}, v\right)=0$ for any $S \in \mathcal{M}(\mathbb{S})$ and $v \in \mathbb{A}$. Also, for $\xi=\check{\xi}, \widehat{\xi}$

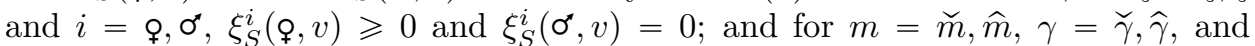
$i, i_{1}, i_{2}=q, \sigma^{\prime \prime}, m_{S}^{i}\left(\sigma^{\prime \prime}, v\right)=\gamma_{S}^{i_{1}, i_{2}}\left(\sigma^{\prime \prime}, v\right)=0$. Writing $S^{\circ}(d v)=S(\{q\}, d v)$ for the age structure of the female subpopulation and $S^{\sigma^{*}}(d v)=S\left(\left\{\sigma^{*}\right\}, d v\right)$ for that of the male subpopulation, we can obtain equations governing the subpopulations. Note

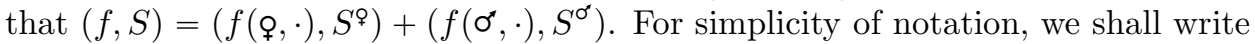

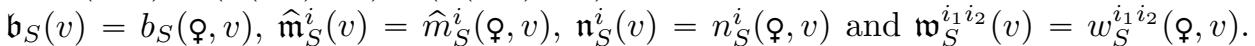
Taking $f(i, v)=f_{\odot}(v) \mathbf{1}_{i=\emptyset}$ in (2) gives the age structure of the female subpopulation:

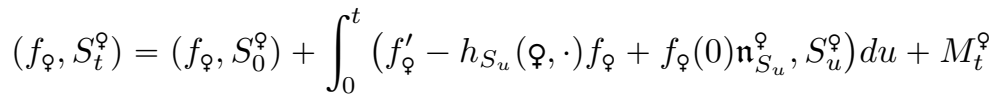

with

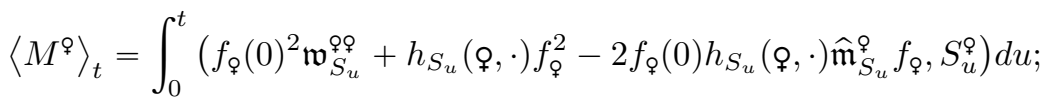

whereas taking $f(i, v)=f_{\sigma^{*}}(v) \mathbf{1}_{i=\sigma^{*}}$ in (2) gives the dynamics of the male subpopulation:

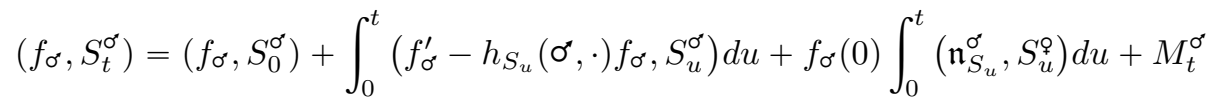

with

$$
\left\langle M^{\sigma^{\prime}}\right\rangle_{t}=f_{\sigma^{\prime}}(0)^{2} \int_{0}^{t}\left(\mathfrak{w}_{S_{u}}^{\sigma^{\circ}}, S_{u}^{\text {\& }}\right) d u+\int_{0}^{t}\left(h_{S_{u}}\left(\sigma^{*}, \cdot\right) f_{\sigma^{*}}^{2}, S_{u}^{\sigma^{*}}\right) d u .
$$

This approach is different from the so-called bisexual branching process, where individuals mate to form couples, which then reproduce like in a Galton-Watson type process in discrete time. Those were introduced by Daley [4] and have been studied by many others (cf. [28] and references therein). A (pseudo) continuous time bisexual branching process, was given in [29], mating supposed to occur at the events of a point process and individuals born by couples having independent and identically distributed life spans. As opposed to that, our model is individual based, and females reproduce, with an intensity, which may depend on the availability of males, among other things. Sexual reproduction without couple formation, like in fish, as well as asexual reproduction, can be easily handled within this framework. 
Couple formation and sexual reproduction with mating, like in many higher animals, can be captured, to some extent, by careful choice of rates and offspring distribution.

2.2. The limit theorems. We consider a family of population processes as above, indexed by some parameter $K \geqslant 1$, which as mentioned, arises from the notion of carrying capacity of the habitat and often can be interpreted as some size where a population neither tends to increase nor decrease systematically. However, it needs not play such a role, and can be viewed just as a natural system scaling parameter, since we consider starting populations whose size is of the order $K$. The purpose is to establish the asymptotic behaviour as $K$ increases, under the assumption that the dynamics of the population depends on $K$ through the reproduction parameters. For notational consistency with limits, we write the parameters in the form $q_{S / K}^{K}(s)$, for $q=b, h, m, \gamma$. The conditions stated for $m$ and $\gamma$ are to be satisfied by all $\breve{m}^{i}$, $\hat{m}^{i}, \breve{\gamma}^{i_{1} i_{2}}$ and $\hat{\gamma}^{i_{1}, i_{2}}$ for any $i, i_{1}, i_{2} \in \mathbb{K}$.

We consider a finite time interval $\mathbb{T}=[0, T]$. Thus, to obtain a bounded age space $\mathbb{A}$, it suffices to assume that the age of the oldest individual over the family of processes at time $0, a^{*}$, is finite:

$$
a^{*}:=\sup _{K \geqslant 1} \sup _{i \in \mathbb{K}}\left(\inf \left\{v>0: S_{0}^{K}(i,(v, \infty))=0\right\}\right)<\infty .
$$

Then the age of the oldest individual in the population at time $t$ cannot exceed $t+a^{*}$, and we can take $\mathbb{A}=[0, \omega]$ with $\omega=T+a^{*}$ as the age space, and $\mathbb{S}=\mathbb{K} \times \mathbb{A}$ the individual state space.

Specifically, we establish the Law of Large Numbers and the Central Limit Theorem associated with our age and type structure process. For a simple case of sexual reproduction (cf. Section 2.1.1) the Law of Large Numbers was stated in [20], without proof. Here, we give and prove results for more general cases. The Law of Large Numbers is stated in Section 2.2.1 with proof in Section A.2, and the Central Limit Theorem is stated in Section 2.2.3 with proof in Section A.3.

2.2.1. Law of Large Numbers. Denote by $\bar{S}_{t}^{K}=S_{t}^{K} / K$ the density of the population. For any $f \in C^{1}(\mathbb{S})$ and $t \in \mathbb{T}$,

$$
\left(f, \bar{S}_{t}^{K}\right)=\left(f, \bar{S}_{0}^{K}\right)+\int_{0}^{t}\left(L_{\bar{S}_{u}^{K}}^{K} f, \bar{S}_{u}^{K}\right) d u+\frac{1}{K} M_{t}^{f, K},
$$

where

$$
L_{S}^{K} f=f^{\prime}-h_{S}^{K} f+\sum_{i \in \mathbb{K}} f(i, 0) n_{S}^{i, K}
$$

and $M_{t}^{f, K}$ is a locally square integrable martingale with predictable quadratic variation

$$
\begin{aligned}
\left\langle M^{f, K}\right\rangle_{t}=\int_{0}^{t}\left(\sum_{i_{1} \in \mathbb{K}} \sum_{i_{2} \in \mathbb{K}} f\left(i_{1}, 0\right) f\left(i_{2}, 0\right) w_{\bar{S}_{u}^{K}}^{i_{1} i_{2}, K}\right. & +h_{\bar{S}_{u}^{K}}^{K} f^{2} \\
& \left.-2 \sum_{i \in \mathbb{K}} f(i, 0) h_{\bar{S}_{u}^{K}}^{K} \hat{m}_{\bar{S}_{u}^{K}}^{i, K} f, S_{u}^{K}\right) d u .
\end{aligned}
$$

We have the Law of Large Numbers when the population process is demographically smooth, i.e. satisfies the following conditions: 
(C0) The model parameters $b, h, m$ and $\gamma$ are uniformly bounded, i.e., for $q=$ $b, h, m, \gamma, \sup q_{\mu}^{K}(s)<\infty$, where supremum is taken over $s \in \mathbb{S}, K \geqslant 1$ and $\mu \in \mathcal{M}(\mathbb{S})$.

(C1) The model parameters $b, h$ and $m$ are normed uniformly Lipschitz in the following sense: for $q=b, h, m$, there exists $c>0$ such that for all $K \geqslant 1$, $\left\|q_{\mu}^{K}-q_{\nu}^{K}\right\|_{\infty} \leqslant c\|\mu-\nu\|$, with $\|\mu\|:=\sup _{\|f\|_{\infty} \leqslant 1, f \text { continuous }}|(f, \mu)|$.

(C2) For $q=b, h, m$, the sequence $q^{K}$ converges (pointwise in $\mu$ and uniformly in $s)$ to $q_{\mu}^{\infty}(s):=\lim _{K \rightarrow \infty} q_{\mu}^{K}(s)$.

(C3) $\bar{S}_{0}^{K}$ converges weakly to $\bar{S}_{0}$ and $\sup _{K}\left(1, \bar{S}_{0}^{K}\right)<\infty$, that is, the process stabilises initially.

Theorem 1. Under the smooth demography conditions (C0)-(C3), the measurevalued process $\bar{S}^{K}$ converges weakly in the Skorokhod space $\mathbb{D}(\mathbb{T}, \mathcal{M}(\mathbb{S}))$, as $K \rightarrow \infty$, to a deterministic measure-valued process $\bar{S}$, satisfying

$$
\left(f, \bar{S}_{t}\right)=\left(f, \bar{S}_{0}\right)+\int_{0}^{t}\left(L_{\bar{S}_{u}}^{\infty} f, \bar{S}_{u}\right) d u
$$

where

$$
L_{S}^{\infty} f=f^{\prime}-h_{S}^{\infty} f+\sum_{i \in \mathbb{K}} f(i, 0) n_{S}^{i, \infty},
$$

for any $f \in C^{1}(\mathbb{S})$ and $t \in \mathbb{T}$.

2.2.2. Relevant spaces and embeddings. Before stating the Central Limit Theorem, we introduce some Sobolev spaces on $\mathbb{S}$ and the relevant duals, where the convergence will take place. The compactness of the space $\mathbb{S}$ allows us to work with the classical Sobolev spaces, instead of the weighted Sobolev spaces like other scholars (e.g. [3, 26, 27]) did.

For $j \in \mathbb{N}_{0}$, let $W^{j}(\mathbb{S})$ be the closure of $C^{\infty}(\mathbb{S})$ with respect to the norm

$$
\|f\|_{W^{j}(\mathbb{S})}=\left(\sum_{\iota=0}^{j} \sum_{i \in \mathbb{K}} \int_{\mathbb{A}}\left(f^{(\iota)}(i, v)\right)^{2} d v\right)^{1 / 2},
$$

where $f^{(\iota)}$ are the (weak) derivatives of $f$. In other words, $W^{j}(\mathbb{S})$ is the set of functions $f$ on $\mathbb{S}$ such that $f$ and its weak derivatives up to order $j$ have a finite $L^{2}(\mathbb{S})$ norm,

$$
\|f\|_{L^{2}(\mathbb{S})}=\left(\sum_{i \in \mathbb{K}} \int_{\mathbb{A}} f(i, v)^{2} d v\right)^{1 / 2} .
$$

The space $W^{j}(\mathbb{S})$ is a Hilbert space. As in [7, we have the following embeddings:

$$
C^{j}(\mathbb{S}) \hookrightarrow W^{j}(\mathbb{S}), W^{j+1}(\mathbb{S}) \hookrightarrow C^{j}(\mathbb{S}) \quad \text { and } \quad W^{j+1}(\mathbb{S}) \underset{H . S .}{\hookrightarrow} W^{j}(\mathbb{S}),
$$

where H.S. stands for Hilbert-Schmidt embedding.

The dual spaces $C^{-j}(\mathbb{S})$ and $W^{-j}(\mathbb{S})$ of $C^{j}(\mathbb{S})$ and $W^{j}(\mathbb{S})$ respectively, are embedded as follows:

$$
W^{-j}(\mathbb{S}) \hookrightarrow C^{-j}(\mathbb{S}), C^{-j}(\mathbb{S}) \hookrightarrow W^{-(j+1)}(\mathbb{S}) \text { and } W^{-j}(\mathbb{S}) \underset{H . S .}{\hookrightarrow} W^{-(j+1)}(\mathbb{S}),
$$

and in particular,

$$
C^{-0}(\mathbb{S}) \hookrightarrow C^{-1}(\mathbb{S}) \hookrightarrow W^{-2}(\mathbb{S}) \hookrightarrow W^{-3}(\mathbb{S}) \underset{H . S .}{\hookrightarrow} W^{-4}(\mathbb{S}) .
$$

The Hilbert-Schmidt embedding plays an important role, since with this, a ball in the smaller space $\left(W^{-3}\right)$ is precompact in the larger space $\left(W^{-4}\right)$. This fact renders 
it possible to prove the coordinate tightness of the fluctuation process. The other embeddings are required due to the derivative in the definition of the operator $L_{S}^{K}$.

For ease of notation, we suppress writing $(\mathbb{S})$ for the spaces from here onwards, that is, we will write $W^{j}$ and $C^{j}$ to mean $W^{j}(\mathbb{S})$ and $C^{j}(\mathbb{S})$. Further, $L^{\infty}$ denotes the set of all bounded measurable functions on $\mathbb{S}=\mathbb{K} \times \mathbb{A}$ with its natural product sigma-algebra $\mathcal{B}(\mathbb{S})$.

2.2.3. The Central Limit Theorem. Let $Z^{K}=\sqrt{K}\left(\bar{S}^{K}-\bar{S}\right)$. Then, for any $f \in C^{1}$ and $t \in \mathbb{T}$,

$$
\left(f, Z_{t}^{K}\right)=\left(f, Z_{0}^{K}\right)+\sqrt{K} \int_{0}^{t}\left(L_{\bar{S}_{u}^{K}}^{K} f-L_{\bar{S}_{u}}^{\infty} f, \bar{S}_{u}\right) d u+\int_{0}^{t}\left(L_{\bar{S}_{u}^{K}}^{K} f, Z_{u}^{K}\right) d u+\tilde{M}_{t}^{f, K},
$$

where $L_{S}^{K}$ and $L_{S}^{\infty}$ are defined as in (5) and (7), and $\tilde{M}_{t}^{f, K}$ is a square integrable martingale with predictable quadratic variation

$$
\begin{aligned}
\left\langle\tilde{M}^{f, K}\right\rangle_{t}=\int_{0}^{t}\left(\sum_{i_{1} \in \mathbb{K}} \sum_{i_{2} \in \mathbb{K}} f\left(i_{1}, 0\right) f\left(i_{2}, 0\right) w_{\bar{S}_{u}^{K}}^{i_{1} i_{2}, K}\right. & +h_{\bar{S}_{u}^{K}}^{K} f^{2} \\
& \left.-2 \sum_{i \in \mathbb{K}} f(i, 0) h_{\bar{S}_{u}^{K}}^{K} \hat{m}_{\bar{S}_{u}^{K}}^{i, K} f, \bar{S}_{u}^{K}\right) d u .
\end{aligned}
$$

In addition to $(\mathrm{C} 0)-(\mathrm{C} 3)$, we impose the following assumptions:

(A0) Conditions (C1) and (C2) hold also for $q=\gamma$.

(A1) $\Xi:=\sup _{i, s, S, K} \breve{\xi}_{S}^{i, K}(s) \vee \sup _{i, s, S, K} \hat{\xi}_{S}^{i, K}(s)$ is square integrable.

(A2) The reproduction parameters $b_{S}^{K}(s), h_{S}^{K}(s)$ and $m_{S}^{K}(s)$ and their limits (in the sense of $(\mathrm{C} 2)$ ) are in the space $C^{4}$ (in the argument $s$ ) with convergence in $C^{4}$. Moreover, $\sqrt{K} \sup _{\mu}\left\|q_{\mu}^{K}-q_{\mu}^{\infty}\right\|_{\infty} \rightarrow 0$ as $K \rightarrow \infty$, $\sup _{K, \mu}\left\|q_{\mu}^{K}\right\|_{C^{3}}<$ $\infty$ and $\sup _{\mu}\left\|q_{\mu}^{\infty}\right\|_{C^{4}}<\infty$, for $q=b, h, m$.

(A3) The limiting parameters (seen as functions of $S$ ) are Fréchet differentiable at every $S$. Namely, for $q=b, h, m$, for every $\mu$, there exists a continuous linear operator $\partial_{S} q_{\mu}^{\infty}: W^{-4} \rightarrow L^{\infty}$ such that

$$
\lim _{\|\nu\|_{W^{-4} \rightarrow 0}} \frac{1}{\|\nu\|_{W^{-4}}}\left\|q_{\mu+\nu}^{\infty}-q_{\mu}^{\infty}-\partial_{S} q_{\mu}^{\infty}(\nu)\right\|_{\infty}=0 .
$$

Moreover, $\sup _{\mu}\left\|\partial_{S} q_{\mu}^{\infty}\right\|_{\mathbb{L}^{-4}} \leqslant c$, where $\mathbb{L}^{-4}=L\left(W^{-4}, L^{\infty}\right)$ denotes the space of continuous linear mappings from $W^{-4}$ to $L^{\infty}$.

(A4) $Z_{0}^{K}$ converges to $Z_{0}$ in $W^{-4}$ and $\sup _{K}\left\|Z_{0}^{K}\right\|_{W^{-2}}<\infty$.

Then the fluctuation process $Z^{K}$ converges.

Theorem 2. Under assumptions (CO)-(C3) and (AO)-(A4), as $K \rightarrow \infty$, the process $\left(Z_{t}^{K}\right)_{t \in \mathbb{T}}$ converges weakly in $\mathbb{D}\left(\mathbb{T}, W^{-4}\right)$ to $\left(Z_{t}\right)_{t \in \mathbb{T}}$ satisfying, for $f \in W^{4}$ and $t \in \mathbb{T}$,

$$
\begin{aligned}
\left(f, Z_{t}\right)=\left(f, Z_{0}\right)+\int_{0}^{t}( & \left.-\partial_{S} h_{\bar{S}_{u}}^{\infty}\left(Z_{u}\right) f+\sum_{i \in \mathbb{K}} f(i, 0) \partial_{S} n_{\bar{S}_{u}}^{i, \infty}\left(Z_{u}\right), \bar{S}_{u}\right) d u \\
& +\int_{0}^{t}\left(f^{\prime}-h_{\bar{S}_{u}}^{\infty} f+\sum_{i \in \mathbb{K}} f(i, 0) n_{\bar{S}_{u}}^{i, \infty}, Z_{u}\right) d u+\tilde{M}_{t}^{f, \infty},
\end{aligned}
$$


where $\tilde{M}_{t}^{f, \infty}$ is a continuous Gaussian martingale with predictable quadratic variation

$\left\langle\tilde{M}^{f, \infty}\right\rangle_{t}=\int_{0}^{t}\left(\sum_{i_{1} \in \mathbb{K}} \sum_{i_{2} \in \mathbb{K}} f\left(i_{1}, 0\right) f\left(i_{2}, 0\right) w_{\bar{S}_{u}}^{i_{1} i_{2}, \infty}+h_{\bar{S}_{u}}^{\infty} f^{2}-2 \sum_{i \in \mathbb{K}} f(i, 0) h_{\bar{S}_{u}}^{\infty} \hat{m}_{\bar{S}_{u}}^{i, \infty} f, \bar{S}_{u}\right) d u$.

For each $t$, the limit $Z_{t}$ takes value in $W^{-4}$. Under certain condition, its expectation corresponds to a signed measure. This is made precise in the following proposition.

Proposition 1. Suppose that $\partial_{S} h_{\mu}^{\infty}(B)(s)$ is of the form $\left(g^{h}(\mu, s, \cdot), B\right)$ where $g^{h}(S, s, \cdot) \in W^{4}$ with $\sup _{S, s}\left\|g^{h}(S, s, \cdot)\right\|_{W^{4}}<\infty$, and similarly, $\partial_{S} n_{\mu}^{i, \infty}(B)(s)$ is of the form $\left(g^{n, i}(\mu, s, \cdot), B\right)$ where $g^{n, i}(S, s, \cdot) \in W^{4}$ with $\sup _{S, s}\left\|g^{n, i}(S, s, \cdot)\right\|_{W^{4}}<\infty$. Then for each $t, \nu_{t}: W^{4} \ni f \mapsto \mathbb{E}\left[\left(f, Z_{t}\right)\right]$ defines a signed measure on $(\mathbb{S}, \mathcal{B}(\mathbb{S}))$.

We give the proofs in Appendices; that of Theorem 1 in Section A.2, that of Theorem 2 in Section A.3, and that of Proposition 1 in Section A.4.

Loosely speaking, the Law of Large Numbers gives the first order approximation to the population when it is large; the Central Limit Theorem gives the second order approximation. Quantities of interest, such as the population size or the number of individuals of certain type within certain age interval, can be computed simply by choosing the appropriate test functions. In specific cases, where explicit model parameters are available, more information can be obtained and inference be made from observations.

2.2.4. Applications: sexual reproduction, revisited. We continue from Section 2.1.1 revising notation slightly, writing parameters in the form $q_{\bar{S}}^{K}(s)$. Suppose that all the conditions (C0)-(C3) and (A0)-(A4) are satisfied, so that the Law of Large Numbers and Central Limit Theorem hold. For example, $q$ may take the form $q(i, v,(1, \bar{S}),(g, \bar{S}))$ with $g(i, v)=\mathbf{1}_{i=q}$. Then, the limiting parameters have the same form and the Fréchet derivatives are

$$
\partial_{S} q_{\mu}^{\infty}(B)(i, v)=\partial_{3} q(i, v,(1, \mu),(g, \mu))(1, B)+\partial_{4} q(i, v,(1, \mu),(g, \mu))(g, B) .
$$

In this case, the conditions on the parameters are satisfied if $\sup \partial_{2}^{j} q(i, x, y, z)<\infty$ for $j=0,1, \ldots, 4, \sup \partial_{3} q(i, x, y, z)<\infty$ and $\sup \partial_{4} q(i, x, y, z)<\infty$, where the supremum is taken over all $i, x, y, z$.

The limiting population density of (6) can be decomposed into two equations, for the limiting female and male measures:

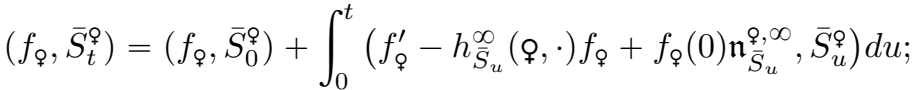

$$
\begin{aligned}
& \left(f_{\sigma^{\circ}}, \bar{S}_{t}^{\sigma^{\prime}}\right)=\left(f_{\sigma^{\prime}}, \bar{S}_{0}^{\sigma^{\sigma}}\right)+\int_{0}^{t}\left(f_{\sigma^{\prime}}^{\prime}-h_{\bar{S}_{u}}^{\infty}\left(\sigma^{\prime \prime}, m \cdot\right) f_{\sigma^{\prime}}, \bar{S}_{u}^{\sigma^{\prime}}\right) d u+f_{\sigma^{\circ}}(0) \int_{0}^{t}\left(\mathfrak{n}_{\bar{S}_{u}^{\circ, \infty}}^{\sigma_{u}}, \bar{S}_{u}^{q}\right) d u .
\end{aligned}
$$

In the case where these measures have densities (with respect to Lebesgue measure), namely $s^{q}(v, t)$ for $\bar{S}_{t}^{q}$ and $s^{\sigma^{\prime}}(v, t)$ for $\bar{S}_{t}^{\sigma^{\circ}}$, the densities resemble the McKendrickvon Foerster equations, as pointed out in [20]:

$$
\begin{aligned}
& \left(\frac{\partial}{\partial t}+\frac{\partial}{\partial v}\right) s^{q}(v, t)=-h_{\bar{S}_{t}}^{\infty}(q, v) s^{q}(v, t), \quad s^{q}(0, t)=\int_{0}^{t+a^{*}} \mathfrak{n}_{\bar{S}_{t}, \infty}(v) s^{q}(v, t) d v, \\
& \left(\frac{\partial}{\partial t}+\frac{\partial}{\partial v}\right) s^{\sigma^{\circ}}(v, t)=-h_{\bar{S}_{t}}^{\infty}\left(\sigma^{*}, v\right) s^{\sigma^{\circ}}(v, t), \quad s^{\sigma^{\circ}}(0, t)=\int_{0}^{t+a^{*}} \mathfrak{n}_{\bar{S}_{t}, \infty}(v) s^{q}(v, t) d v .
\end{aligned}
$$


The upper limit in these integrals is due to $s^{q}(v, t)=0$ for $v>t+a^{*}$.

The fluctuation limit (9) can be decomposed as follows, with $Z^{\circ}(d v)=Z(\{q\}, d v)$ and $Z^{\sigma^{*}}(d v)=Z\left(\left\{\sigma^{\prime}\right\}, d v\right)$,

$$
\begin{aligned}
& \left(f_{q}, Z_{t}^{q}\right)=\left(f_{q}, Z_{0}^{q}\right)+\int_{0}^{t}\left(-\partial_{S} h_{\bar{S}_{u}}^{\infty}\left(Z_{u}\right)(q, \cdot) f_{q}+f_{q}(0) \partial_{S} \mathfrak{n}_{\bar{S}_{u}, \infty}\left(Z_{u}\right), \bar{S}_{u}^{\propto}\right) d u \\
& +\int_{0}^{t}\left(f_{q}^{\prime}-h_{\bar{S}_{u}}^{\infty}(\propto, \cdot) f_{q}+f_{q}(0) \mathfrak{n}_{\bar{S}_{u}, \infty}, Z_{u}^{\rho}\right) d u+\tilde{M}_{t}^{q},
\end{aligned}
$$

where

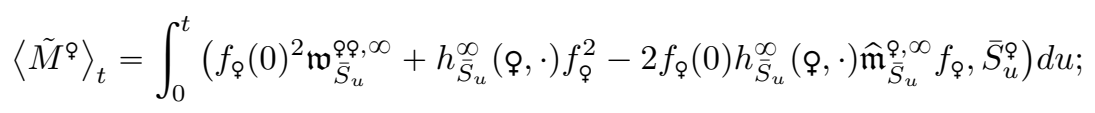

and

$$
\begin{aligned}
& \left(f_{\sigma^{\prime}}, Z_{t}^{\sigma^{\circ}}\right)=\left(f_{\sigma^{\circ}}, Z_{0}^{\sigma^{\circ}}\right)+\int_{0}^{t}\left(-\partial_{S} h_{\bar{S}_{u}}^{\infty}\left(Z_{u}\right)\left(\sigma^{\star}, \cdot\right) f_{\sigma^{\prime}}, \bar{S}_{u}^{\sigma^{\circ}}\right) d u \\
& +f_{\sigma^{\circ}}(0) \int_{0}^{t}\left(\partial_{S} \mathfrak{n}_{\bar{S}_{u}, \infty}^{\sigma_{u}}\left(Z_{u}\right), \bar{S}_{u}^{o}\right) d u+\int_{0}^{t}\left(f_{\sigma^{\prime}}^{\prime}-h_{\bar{S}_{u}}^{\infty}\left(\sigma^{\prime \prime}, \cdot\right) f_{\sigma^{\prime}}, Z_{u}^{\sigma^{\circ}}\right) d u \\
& +f_{\sigma^{\prime}}(0) \int_{0}^{t}\left(\mathfrak{n}_{\bar{S}_{u}^{\circ}, \infty}^{\sigma^{\prime}}, Z_{u}^{\propto}\right) d u+\tilde{M}_{t}^{\sigma^{\circ}},
\end{aligned}
$$

where

$$
\left\langle\tilde{M}^{\sigma^{\circ}}\right\rangle_{t}=\int_{0}^{t}\left(f_{\sigma^{\circ}}(0)^{2} \mathfrak{w}_{\bar{S}_{u}}^{\sigma^{\circ}, \infty}, \bar{S}_{u}^{\Phi}\right) d u+\int_{0}^{t}\left(h_{\bar{S}_{u}}^{\infty}\left(\sigma^{\prime \prime}, \cdot\right) f_{\sigma^{\prime}}^{2}, \bar{S}_{u}^{\sigma^{\circ}}\right) d u .
$$

The McKendrick-von Foerster equations in their turn may take many special forms, in different situations, dependent upon the varying fertilisation, reproduction and survival patterns, as well as the role of the carrying capacity type parameter. It is, however, important that such modelling be done in close relationship to the biological circumstances at hand. In this broad context, we contend ourselves with the remark that typically female fertility will increase with the abundance of males while mortality on the whole will tend to increase with competition and, thus, population size. We shall not enter into the intricate details of this, like different forms of Allee effects about which there is a rich literature, e.g. [6] and [9].

2.3. With migration. Migration can be easily incorporated into the model. While emigrations can be seen and taken as deaths, immigrations can be formulated as individuals of different ages and types arriving at random times. Henceforth, immigrations will be our concern. In the case of controlled immigration, that is, when the rate of immigration and the number of immigrants per arrival are bounded, we can show that the limits for the Law of Large Numbers and Central Limit Theorem coincide with those without immigration.

Suppose that immigrations occur at random times $\tilde{\tau}_{1}, \tilde{\tau}_{2}, \ldots$ with rate $g\left(S_{u}, u\right)$, depending on the population composition and time. At time $\tilde{\tau}_{j}, \zeta_{j}$ new immigrants arrive with $\mathbb{E}\left[\zeta_{j} \mid \tilde{\tau}_{j}, S_{\tilde{\tau}_{j}}\right]=\tilde{m}\left(S_{\tilde{\tau}_{j}}, \tilde{\tau}_{j}\right)$ and $\mathbb{E}\left[\zeta_{j}^{2} \mid \tilde{\tau}_{j}, S_{\tilde{\tau}_{j}}\right]=\tilde{v}\left(S_{\tilde{\tau}_{j}}, \tilde{\tau}_{j}\right)$. The type and age of each immigrant is random, distributed according to some distribution $\tilde{\mathcal{K}}\left(\cdot ; S_{\tilde{\tau}_{j}}, \tilde{\tau}_{j}\right)$ conditioning on the arrival time and the population structure at that time. In other words, if $x$ is an individual who immigrated at time $u$, denote its type and age at immigration by $\kappa_{x}$ and $\tilde{\alpha}_{x}$ respectively, then $\mathbb{P}\left(\kappa_{x}=i, \tilde{\alpha}_{x} \in A\right)=$ $\int_{\{i\} \times A} \tilde{\mathcal{K}}\left(d s ; S_{u}, u\right)$. 
Let $G((i, v), t)$ be the number of immigrants by time $t$ with type $i$ and age at immigration not more than $v$. Then, the equation analogous to (14) is

$$
\begin{aligned}
\left(f, S_{t}\right)=\left(f, S_{0}\right)+\int_{0}^{t}\left(f^{\prime}, S_{u}\right) d u & +\sum_{i \in \mathbb{K}} f(i, 0) B^{i}([0, t]) \\
& -\int_{\mathbb{S} \times[0, t]} f(s) D(d s, d u)+\int_{\mathbb{S} \times[0, t]} f(s) G(d s, d u) .
\end{aligned}
$$

Compensating the above, we obtain a dynamical equation in the form of a semimartingale decomposition:

$$
\begin{aligned}
\left(f, S_{t}\right)=\left(f, S_{0}\right)+\int_{0}^{t}\left(L_{S_{u}} f\right. & \left.S_{u}\right) d u \\
& \quad+\int_{0}^{t} g\left(S_{u}, u\right) \tilde{m}\left(S_{u}, u\right) \int_{\mathbb{S}} f(s) \tilde{\mathcal{K}}\left(d s ; S_{u}, u\right) d u+\dddot{M}_{t}^{f},
\end{aligned}
$$

where $L_{S} f$ is as in (2) and $\dddot{M}_{t}^{f}$ is a locally square integrable martingale with predictable quadratic variation

$$
\begin{aligned}
\left\langle\dddot{M}^{f}\right\rangle_{t}=\int_{0}^{t}\left(\sum_{i_{1} \in \mathbb{K}} \sum_{i_{2} \in \mathbb{K}} f\left(i_{1}, 0\right) f\left(i_{2}, 0\right) w_{S_{u}}^{i_{1} i_{2}}+h_{S_{u}} f^{2}-2 \sum_{i \in \mathbb{K}} f(i, 0) h_{S_{u}} \hat{m}_{S_{u}}^{i} f, S_{u}\right) d u \\
+\int_{0}^{t} g\left(S_{u}, u\right) \\
+\tilde{m}\left(S_{u}, u\right) \int_{\mathbb{S}} f^{2}(s) \tilde{\mathcal{K}}\left(d s ; S_{u}, u\right) \\
\left.+\left(\tilde{v}\left(S_{u}, u\right)-\tilde{m}\left(S_{u}, u\right)\right)\left(\int_{S} f(s) \tilde{\mathcal{K}}\left(d s ; S_{u}, u\right)\right)^{2}\right\} d u
\end{aligned}
$$

Suppose that $g, \tilde{m}$ and $\tilde{v}$ are bounded. Then the Law of Large Numbers and the Central Limit Theorem hold, and the limits are the same as the corresponding limits in the case without immigration.

\section{Sexual Reproduction in Serial monogamy mating System}

Our framework can be applied immediately to model sexual reproduction, as in Sections 2.1.1 and 2.2.4. However, the formulation sketched there does not take the form of mating into account. In this section, we adapt our framework to model a population with serial monogamy. In other words, a female and a male form a couple for life or for some time, like a breeding season.

3.1. The model. Despite not restricting the model to human population, for the convenience of terminology, we refer to the formation of a couple as a "marriage". Consider a population consisting of individuals of three types; type 1 (denoted as \$) refers to single females, type 2 (denoted as o") refers to single males and type 3 (denoted as $థ$ ) refers to "married" couples. An individual from type $\$$ and an individual from type $\sigma^{\prime \prime}$ can form a couple (get married) and becomes a type $\$$. A couple lasts a period of random length interrupted by the death of one of the mates, in which case, the survivor becomes single and available for mating again.

A type $\Phi^{\prime}$ gives birth at random times to random number of type $q$ and type $\sigma$ offspring. We assume also the possibility of a type $q$ to give birth. The lifetime of each individual is random.

Let $\boldsymbol{q}$ (resp. $\bullet$ ) denote the set of all females (resp. males), single or married. Let $c_{x, y}$ denote the time individuals $x$ and $y$ become a couple, $c_{x}^{j}$ denote the time 
of the $j$ th "marriage" of individual $x$ and $d_{x}^{j}$ denote the time $x$ becomes a "widow" from its $j$ th marriage. To accomodate the notation for couples, the age of which consists of both that of the female partner and the male partner, we assign also an "age" of two indices to each individual that is single, with one index taking value $\infty$. Then, the age structure at time $t$ of the three types are given by

$$
\begin{aligned}
& S_{t}^{\odot}(d i, d v, d w)=\sum_{x \in \boldsymbol{\varphi}} \mathbf{1}_{\tau_{x} \leqslant t \leqslant \sigma_{x}}\left(\mathbf{1}_{t \leqslant c_{x}^{1}}+\mathbf{1}_{d_{x}^{1} \leqslant t \leqslant c_{x}^{2}}+\mathbf{1}_{d_{x}^{2} \leqslant t \leqslant c_{x}^{3}}+\cdots\right) \delta_{\left(\wp, t-\tau_{x}, \infty\right)}, \\
& S_{t}^{\sigma^{\circ}}(d i, d v, d w)=\sum_{y \in \bullet} \mathbf{1}_{\tau_{y} \leqslant t \leqslant \sigma_{y}}\left(\mathbf{1}_{t \leqslant c_{y}^{1}}+\mathbf{1}_{d_{y}^{1} \leqslant t \leqslant c_{y}^{2}}+\mathbf{1}_{d_{y}^{2} \leqslant t \leqslant c_{y}^{3}}+\cdots\right) \delta_{\left(\sigma^{\circ}, \infty, t-\tau_{y}\right)}, \\
& S_{t}^{\Phi^{\sigma}}(d i, d v, d w)=\sum_{x \in \boldsymbol{\varphi}} \sum_{y \in \bullet} \mathbf{1}_{\tau_{x} \leqslant t \leqslant \sigma_{x}} \mathbf{1}_{\tau_{y} \leqslant t \leqslant \sigma_{y}} \mathbf{1}_{c_{x, y} \leqslant t} \delta_{\left(\varphi^{\prime}, t-\tau_{x}, t-\tau_{y}\right)},
\end{aligned}
$$

and the structure of the entire population

$$
S_{t}(d i, d v, d w)=S_{t}^{\odot}(d i, d v, d w)+S_{t}^{\sigma^{\circ}}(d i, d v, d w)+S_{t}^{\Phi^{\circ}}(d i, d v, d w)
$$

Let $b_{S}(v, w)$ denote the bearing rate of a couple with a female at age $v$ and a male at age $w$, when the population composition is $S$. At each birth, the number of females and the number of males born have the distributions of $\xi_{S}^{\text {}}(v, w)$ and $\xi_{S}^{\sigma^{*}}(v, w)$ respectively. Write $m_{S}^{i}(v, w)=\mathbb{E}\left[\xi_{S}^{i}(v, w)\right]$ and $\gamma_{S}^{i j}(v, w)=\mathbb{E}\left[\xi_{S}^{i}(v, w) \xi_{S}^{j}(v, w)\right]$. These quantities with $w=\infty$ correspond to those of single females.

Let $\rho_{S}(v, w)$ be the rate at which a single female of age $v$ and a single male of age $w$ marry to each other. In other words, for $x \in \boldsymbol{q}$ and $y \in \boldsymbol{\bullet}$, with $d_{x}^{0}:=0=: d_{y}^{0}$,

$$
\mathbf{1}_{c_{x, y} \leqslant t}-\int_{0}^{t \wedge c_{x, y}} \rho_{S_{u}}\left(u-\tau_{x}, u-\tau_{y}\right) \mathbf{1}_{\tau_{x} \leqslant u<\sigma_{x}} \mathbf{1}_{\tau_{y} \leqslant u<\sigma_{y}} \sum_{j \geqslant 0} \mathbf{1}_{d_{x}^{j} \leqslant u \leqslant c_{x}^{j+1}} \sum_{k \geqslant 0} \mathbf{1}_{d_{y}^{k} \leqslant u \leqslant c_{y}^{k+1}} d u
$$

is a martingale. Suppose that marriage lasts for a random length, at rate $h_{S}^{\Phi}(v, w)$ it breaks, dependent upon the ages of the mates as well as the population composition.

Let $h_{S}^{o}(v, w)$ denote the death rate of a female when she is at age $v$ and her partner is at age $w$, when the population composition is $S$. Similarly, let $h_{S}^{\sigma^{*}}(v, w)$ denote the death rate of a male when he is at age $w$ and his partner is at age $v$, when the population composition is $S$. For single females and males, their death rates are $h_{S}^{\circ}(v, \infty)$ and $h_{S}^{\sigma}(\infty, w)$, respectively.

We consider test functions $f: \mathbb{K} \times \mathbb{A} \cup\{\infty\} \times \mathbb{A} \cup\{\infty\} \rightarrow \mathbb{R}$, with $\mathbb{K}=\{1,2,3\} \equiv$ $\left\{o, \sigma^{\prime \prime}, \varsigma^{\prime}\right\}$, such that $f(3, v, w) \equiv f\left(\sigma^{\prime}, v, w\right), f(1, v, w) \equiv f(o, v, w)=f(o, v, \infty)=$ $f_{\phi}(v)$ and $f(2, v, w) \equiv f\left(\sigma^{\prime}, v, w\right)=f\left(\sigma^{\prime}, \infty, w\right)=f_{\sigma^{\prime}}(w)$, for any $v, w \in \mathbb{A}$, for some functions $f_{\odot}$ and $f_{\sigma^{*}}$. We assume that $f(i, v, w)$ is differentiable with respect to $v$

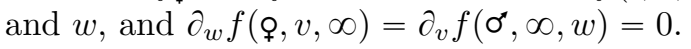


Then, it can be shown that (see Appendix B.1) $S$ has the following semimartingale representation!

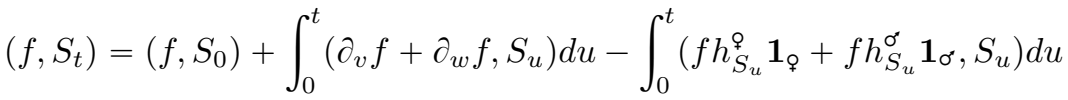

$$
\begin{aligned}
& +\int_{0}^{t}\left(\left(\left(f\left(\sigma^{\prime \prime}, \infty, \cdot\right)-f\left(\Phi^{\prime \prime}, \cdot, \cdot\right)\right) h_{S_{u}}^{\wp}+\left(f(\propto, \cdot, \infty)-f\left(\phi^{\prime \prime}, \cdot, \cdot\right)\right) h_{S_{u}}^{\sigma^{\prime}}\right) \mathbf{1}_{\phi^{\prime}}, S_{u}\right) d u \\
& +\int_{0}^{t}\left(\left(f(\propto, \cdot, \infty)+f\left(\sigma^{\prime \prime}, \infty, \cdot\right)-f\left(\varsigma^{\prime}, \cdot, \cdot\right)\right) h_{S_{u}}^{\phi^{\prime}} \mathbf{1}_{\phi}, S_{u}\right) d u \\
& +\int_{0}^{t}\left(\left[f(\propto, 0, \infty) b_{S_{u}} m_{S_{u}}^{\propto}+f\left(\sigma^{\prime}, \infty, 0\right) b_{S_{u}} m_{S_{u}}^{\sigma^{\star}}\right]\left(\mathbf{1}_{ф}+\mathbf{1}_{\wp}\right), S_{u}\right) d u \\
& -\int_{0}^{t}\left(\left(\left[f(\propto, \cdot, \infty)+f\left(\sigma^{\prime}, \infty, *\right)-f\left(\varsigma^{*}, \cdot, *\right)\right] \rho_{S_{u}}(\cdot, *) \mathbf{1}_{\wp}, S_{u}\right) . \mathbf{1}_{\sigma^{*}}, S_{u}\right)_{*} d u \\
& +M_{t}^{f} \text {, }
\end{aligned}
$$

where $M^{f}$ is a martingale with predictable quadratic variation

$$
\begin{aligned}
& \left\langle M^{f}\right\rangle_{t}=\int_{0}^{t}\left(f^{2} h_{S_{u}}^{\wp} \mathbf{1}_{\odot}+f^{2} h_{S_{u}}^{\sigma_{u}} \mathbf{1}_{\sigma^{*}}, S_{u}\right) d u \\
& +\int_{0}^{t}\left(\left(\left[f(\propto, \cdot, \infty)-f\left(\Phi^{\prime}, \cdot, \cdot\right)\right]^{2} h_{S_{u}}^{\sigma^{\prime}}+\left[f\left(\sigma^{\prime \prime}, \infty, \cdot\right)-f\left(\varsigma^{\prime \prime}, \cdot, \cdot\right)\right]^{2} h_{S_{u}}^{q}\right) \mathbf{1}_{\phi^{\prime}}, S_{u}\right) d u \\
& +\int_{0}^{t}\left(\left(f(\propto, \cdot, \infty)+f\left(\sigma^{\prime \prime}, \infty, \cdot\right)-f\left(\varsigma^{\prime}, \cdot, \cdot\right)\right)^{2} h_{S_{u}}^{\infty} \mathbf{1}_{\phi}, S_{u}\right) d u \\
& +\int_{0}^{t}\left(\left[f^{2}(\propto, 0, \infty) \gamma_{S_{u}}^{\text {\% }}+f^{2}\left(\sigma^{\prime \prime}, \infty, 0\right) \gamma_{S_{u}}^{\sigma^{\prime \prime}}+2 f(\propto, 0, \infty) f\left(\sigma^{\prime \prime}, \infty, 0\right) \gamma_{S_{u}}^{\ddagger}\right] b_{S_{u}}\left(\mathbf{1}_{\phi \circ}+\mathbf{1}_{\wp}\right), S_{u}\right) d u \\
& +\int_{0}^{t}\left(\left(\left[f(\%, \cdot, \infty)+f\left(\sigma^{*}, \infty, *\right)-f\left(\Phi^{*}, \cdot, *\right)\right]^{2} \rho_{S_{u}}(\cdot, *) \mathbf{1}_{\wp}, S_{u}\right) . \mathbf{1}_{\sigma^{*}}, S_{u}\right)_{*} d u \text {. }
\end{aligned}
$$

Considering a family of the population processes indexed by $K \geqslant 1$ and writing the reproduction parameters in the form $q_{S / K}^{K}(\cdot)$, we obtain the asymptotics as $K \rightarrow \infty$.

\footnotetext{
${ }^{1}$ We write $((f(*, \cdot), \mu) ., \nu) *$ to mean $\iint f(x, y) \mu(d y) \nu(d x)=(f, \nu \otimes \mu)$.
} 
3.2. Law of Large Numbers. Let $\bar{S}_{t}^{K}=S_{t}^{K} / K$. Then the scaled process satisfies

$$
\begin{aligned}
& \left(f, \bar{S}_{t}^{K}\right)=\left(f, \bar{S}_{0}^{K}\right)+\int_{0}^{t}\left(\partial_{v} f+\partial_{w} f, \bar{S}_{u}^{K}\right) d u-\int_{0}^{t}\left(f h_{\bar{S}_{u}^{K}}^{\mathcal{O}^{K}} \mathbf{1}_{\boldsymbol{q}}+f h_{\bar{S}_{u}^{K}}^{\sigma^{K}} \mathbf{1}_{\sigma^{\sigma}}, \bar{S}_{u}^{K}\right) d u
\end{aligned}
$$

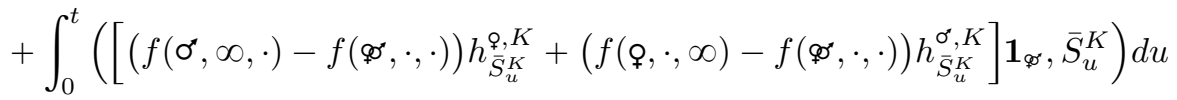

$$
\begin{aligned}
& +\int_{0}^{t}\left(\left(f(\propto, \cdot, \infty)+f\left(\sigma^{\prime \prime}, \infty, \cdot\right)-f\left(\wp^{\prime}, \cdot, \cdot\right)\right) h_{\bar{S}_{u}^{K}}^{\wp, K} \mathbf{1}_{\Phi^{\prime}}, \bar{S}_{u}^{K}\right) d u
\end{aligned}
$$

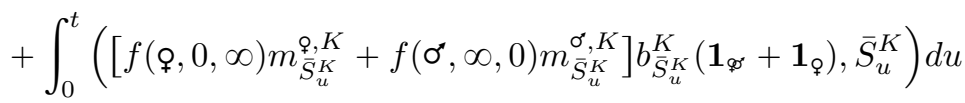

$$
\begin{aligned}
& -\int_{0}^{t}\left(\left(\left[f(\propto, \cdot, \infty)+f\left(\sigma^{*}, \infty, *\right)-f\left(\Phi^{\prime}, \cdot, *\right)\right] K \rho_{\bar{S}_{u}^{K}}^{K}(\cdot, *) \mathbf{1}_{\varphi}, \bar{S}_{u}^{K}\right) . \mathbf{1}_{\sigma^{\prime}}, \bar{S}_{u}^{K}\right)_{*} d u \\
& +\bar{M}_{t}^{f, K} \text {, }
\end{aligned}
$$

with

$$
\begin{aligned}
& \left\langle\bar{M}^{f, K}\right\rangle_{t}=\int_{0}^{t} \frac{1}{K}\left(f^{2} h_{\bar{S}_{u}^{K}}^{\varphi, K} \mathbf{1}_{\uparrow}+f^{2} h_{\bar{S}_{u}^{K}}^{\sigma^{\prime} K} \mathbf{1}_{\sigma^{\prime}}, \bar{S}_{u}^{K}\right) d u \\
& +\int_{0}^{t} \frac{1}{K}\left(\left[\left(f\left(\sigma^{\prime \prime}, \infty, \cdot\right)-f\left(\Phi^{\prime}, \cdot, \cdot\right)\right)^{2} h_{\bar{S}_{u}^{K}}^{\varphi}+\left(f(\propto, \cdot, \infty)-f\left(\Phi^{\prime}, \cdot, \cdot\right)\right)^{2} h_{\bar{S}_{u}^{K}}^{\sigma^{K}}\right] \mathbf{1}_{\phi^{\prime}}, \bar{S}_{u}^{K}\right) d u \\
& +\int_{0}^{t} \frac{1}{K}\left(\left(f(q, \cdot, \infty)+f\left(\sigma^{\prime \prime}, \infty, \cdot\right)-f\left(\Phi^{\prime \prime}, \cdot, \cdot\right)\right)^{2} h_{\bar{S}_{u}^{K}}^{\Phi, \mathbf{1}_{\varphi^{\prime}}}, \bar{S}_{u}^{K}\right) d u
\end{aligned}
$$

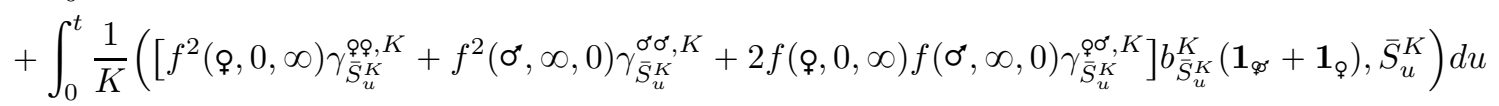

$$
\begin{aligned}
& +\int_{0}^{t}\left(\left(\left[f(\%, \cdot, \infty)+f\left(\sigma^{\prime \prime}, \infty, *\right)-f\left(\infty^{\prime}, \cdot, *\right)\right]^{2} \rho_{\bar{S}_{u}^{K}}^{K}(\cdot, *) \mathbf{1}_{\varphi}, \bar{S}_{u}^{K}\right) . \mathbf{1}_{\sigma^{*}}, \bar{S}_{u}^{K}\right)_{*} d u \text {. }
\end{aligned}
$$

For the convergence of the sequence of processes $\bar{S}^{K}$, we need conditions like (C0) to (C3), with conditions on $\rho$ too.

(C0') In addition to $(\mathrm{C} 0), K \rho^{K}$ is bounded.

(C1') (C1) holds also for $q^{K}$ being $K \rho^{K}$.

(C2') (C2) holds, and $\lim _{K \rightarrow \infty} K \rho_{\mu}^{K}=: \rho_{\mu}^{\infty}$.

(C3') Same as (C3).

Theorem 3. Under the smooth demography conditions (CO')-(C3'), the scaled process $\bar{S}^{K}$ converges weakly in the Skorokhod space $\mathbb{D}(\mathbb{T}, \mathcal{M}(\mathbb{S}))$, as $K \rightarrow \infty$, to a 
deterministic measure-valued process $\bar{S}$, satisfying

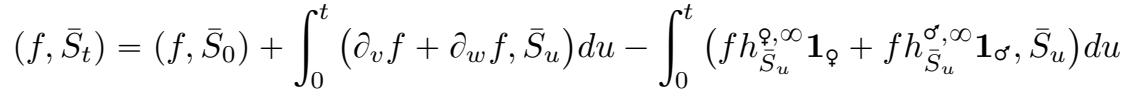

$$
\begin{aligned}
& +\int_{0}^{t}\left(\left[\left(f\left(\sigma^{\prime \prime}, \infty, \cdot\right)-f\left(\Phi^{\prime}, \cdot, \cdot\right)\right) h_{\bar{S}_{u}, \infty}+\left(f(\propto, \cdot, \infty)-f\left(\phi^{\prime \prime}, \cdot, \cdot\right)\right) h_{\bar{S}_{u}, \infty}^{\sigma^{\prime}}\right] \mathbf{1}_{\phi^{\prime}}, \bar{S}_{u}\right) d u \\
& +\int_{0}^{t}\left(\left(f(\propto, \cdot, \infty)+f\left(\sigma^{\prime}, \infty, \cdot\right)-f\left(\varsigma^{\prime}, \cdot, \cdot\right)\right) h_{\bar{S}_{u}, \infty} \mathbf{1}_{\phi}, \bar{S}_{u}\right) d u \\
& +\int_{0}^{t}\left(\left[f(\propto, 0, \infty) m_{\bar{S}_{u}, \infty}+f\left(\sigma^{\prime \prime}, \infty, 0\right) m_{\bar{S}_{u}, \infty}^{\sigma^{*}}\right] b_{\bar{S}_{u}}^{\infty}\left(\mathbf{1}_{\phi}+\mathbf{1}_{\uparrow}\right), \bar{S}_{u}\right) d u \\
& -\int_{0}^{t}\left(\left(\left[f(\propto, \cdot, \infty)+f\left(\sigma^{*}, \infty, *\right)-f(\propto, \cdot, *)\right] \rho_{\bar{S}_{u}}^{\infty}(\bullet, *) \mathbf{1}_{\odot}, \bar{S}_{u}\right) . \mathbf{1}_{\sigma^{*}}, \bar{S}_{u}\right)_{*} d u \text {. }
\end{aligned}
$$

From this, we obtain a system of PDEs for the age densities of the three subpopulations. Suppose $a^{q}(v, t), a^{\sigma^{\sigma}}(w, t)$ and $a^{\Phi}(v, w, t)$ are the densities (in $v$ and $w)$ of $\bar{S}_{t}^{i}, i=\wp, \sigma^{\prime}, \Phi^{\prime}$, respectively. Then the densities satisfy the following:

$$
\begin{aligned}
& \partial_{t} a^{q}(v, t)+\partial_{v} a^{\uparrow}(v, t)=-h_{\bar{S}_{t}}^{\circ, \infty}(v, \infty) a^{\uparrow}(v, t) \\
& +\int\left(h_{\bar{S}_{t}, \infty}^{\sigma^{\infty}}(v, w)+h_{\bar{S}_{t}}^{\Phi, \infty}(v, w)\right) a^{\Phi}(v, w, t) d w-\int \rho_{\bar{S}_{t}}^{\infty}(v, w) a^{\sigma^{\star}}(w, t) d w a^{q}(v, t), \\
& \partial_{t} a^{\sigma^{\prime \prime}}(w, t)+\partial_{w} a^{\sigma^{\prime \prime}}(w, t)=-h_{\bar{S}_{t}, \infty}^{\sigma^{*}}(\infty, w) a^{\sigma^{\prime \prime}}(w, t) \\
& +\int\left(h_{\bar{S}_{t}}^{\circ, \infty}(v, w)+h_{\bar{S}_{t}}^{\Phi, \infty}(v, w)\right) a^{\Phi}(v, w, t) d v-\int \rho_{\bar{S}_{t}}^{\infty}(v, w) a^{q}(v, t) d v a^{\sigma^{\circ}}(w, t), \\
& \partial_{t} a^{\phi s}(v, w, t)+\partial_{v} a^{\phi}(v, w, t)+\partial_{w} a^{\Phi}(v, w, t)=
\end{aligned}
$$

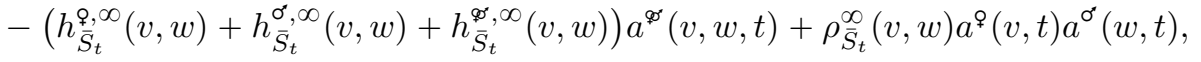

with boundary conditions

$$
\begin{aligned}
& a^{q}(0, t)=\iint m_{\bar{S}_{t}}^{\propto, \infty}(v, w) b_{\bar{S}_{t}}^{\infty}(v, w) a^{థ}(v, w, t) d v d w+\int m_{\bar{S}_{t}, \infty}(v, \infty) b_{\bar{S}_{t}}^{\infty}(v, \infty) a^{q}(v, t) d v, \\
& a^{\sigma^{\circ}}(0, t)=\iint m_{\bar{S}_{t}}^{\sigma^{\infty}, \infty}(v, w) b_{\bar{S}_{t}}^{\infty}(v, w) a^{\Phi}(v, w, t) d v d w+\int m_{\bar{S}_{t}}^{\sigma^{\infty}, \infty}(v, \infty) b_{\bar{S}_{t}}^{\infty}(v, \infty) a^{\ddagger}(v, t) d v .
\end{aligned}
$$

This is comparable to that given by Fredrickson in [8]. 
3.3. Central Limit Theorem. Let $Z^{K}=\sqrt{K}\left(\bar{S}^{K}-\bar{S}\right)$. Then, for any $f \in C^{1}$ and $t \in \mathbb{T}$,

$$
\begin{aligned}
& \left(f, Z_{t}^{K}\right)=\left(f, Z_{0}^{K}\right)+\int_{0}^{t}\left(\partial_{v} f+\partial_{w} f, Z_{u}^{K}\right) d u
\end{aligned}
$$

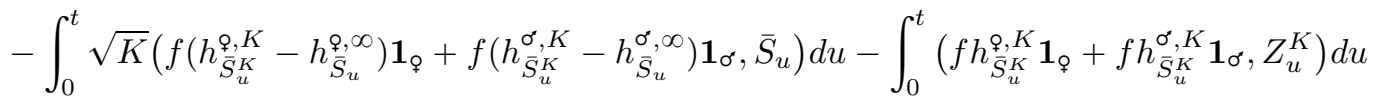

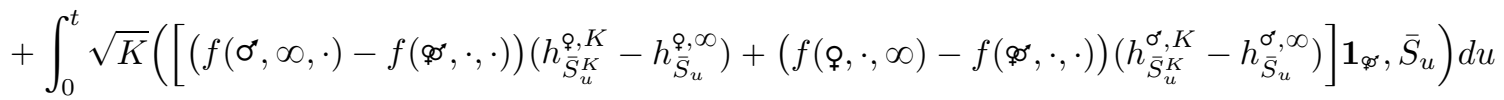

$$
\begin{aligned}
& +\int_{0}^{t}\left(\left[\left(f\left(\sigma^{\prime \prime}, \infty, \cdot\right)-f\left(\Phi^{\prime}, \cdot, \cdot\right)\right) h_{\bar{S}_{u}^{K}}^{\phi, K}+\left(f(\phi, \cdot, \infty)-f\left(\Phi^{K}, \cdot, \cdot\right)\right) h_{\bar{S}_{u}^{K}}^{\sigma^{K}, K}\right] \mathbf{1}_{\phi^{\circ}}, Z_{u}^{K}\right) d u \\
& +\int_{0}^{t} \sqrt{K}\left(\left(f(q, \cdot, \infty)+f\left(\sigma^{\prime}, \infty, \cdot\right)-f\left(\Phi^{\infty}, \cdot, \cdot\right)\right)\left(h_{\bar{S}_{u}^{K}}^{\Phi^{K}}-h_{\bar{S}_{u}, \infty}^{,^{\prime}}\right) \mathbf{1}_{\phi}, \bar{S}_{u}\right) d u \\
& +\int_{0}^{t}\left(\left(f(\propto, \cdot, \infty)+f\left(\sigma^{\star \prime}, \infty, \cdot\right)-f\left(\Phi^{\infty}, \cdot, \cdot\right)\right) h_{\bar{S}_{u}^{K}}^{\wp, K} \mathbf{1}_{\phi}, Z_{u}^{K}\right) d u \\
& +\int_{0}^{t} \sqrt{K}\left(\left[f(\wp, 0, \infty)\left(b_{\bar{S}_{u}^{K}}^{K} m_{\bar{S}_{u}^{K}}^{\wp, K}-b_{\bar{S}_{u}}^{\infty} m_{\bar{S}_{u}}^{\wp, \infty}\right)+f\left(\sigma^{\star}, \infty, 0\right)\left(b_{\bar{S}_{u}^{K}}^{K} m_{\bar{S}_{u}^{K}}^{\sigma^{K}}-b_{\bar{S}_{u}}^{\infty} m_{\bar{S}_{u}, \infty}^{\sigma^{\prime}}\right)\right]\left(\mathbf{1}_{\varphi}+\mathbf{1}_{q}\right), \bar{S}_{u}\right) d u \\
& +\int_{0}^{t}\left(\left[f(\propto, 0, \infty) m_{\bar{S}_{u}^{K}}^{\varphi, K}+f\left(\sigma^{\star \prime}, \infty, 0\right) m_{\bar{S}_{u}^{K}}^{\sigma^{K}, K}\right] b_{\bar{S}_{u}^{K}}^{K}\left(\mathbf{1}_{\Phi}+\mathbf{1}_{\varphi}\right), Z_{u}^{K}\right) d u \\
& -\int_{0}^{t} \sqrt{K}\left(\left(\left[f(q, \cdot, \infty)+f\left(\sigma^{\prime \prime}, \infty, *\right)-f\left(\Phi^{\prime}, \cdot, *\right)\right]\left(K \rho_{\bar{S}_{u}^{K}}^{K}(\cdot, *)-\rho_{\bar{S}_{u}}^{\infty}(\cdot, *)\right) \mathbf{1}_{\varphi}, \bar{S}_{u}\right) . \mathbf{1}^{*}, \bar{S}_{u}\right)_{*} d u \\
& -\int_{0}^{t}\left(\left(\left[f(\wp, \cdot, \infty)+f\left(\sigma^{*}, \infty, *\right)-f\left(\varsigma^{\prime}, \cdot, *\right)\right] K \rho_{\bar{S}_{u}^{K}}^{K}(\cdot, *) \mathbf{1}_{\varphi}, Z_{u}^{K}\right) . \mathbf{1}_{\sigma^{\prime}}, \bar{S}_{u}\right)_{*} d u \\
& -\int_{0}^{t}\left(\left(\left[f(\varphi, \cdot, \infty)+f\left(\sigma^{\prime \prime}, \infty, *\right)-f\left(\infty^{\prime}, \cdot, *\right)\right] K \rho_{\bar{S}_{u}^{K}}^{K}(\cdot, *) \mathbf{1}_{\varphi}, \bar{S}_{u}\right) . \mathbf{1}_{\sigma^{*}}, Z_{u}^{K}\right)_{*} d u \\
& +\tilde{M}_{t}^{f, K}
\end{aligned}
$$

where $\tilde{M}_{t}^{f, K}$ is a square integrable martingale with predictable quadratic variation

$$
\begin{aligned}
& \left\langle\tilde{M}^{f, K}\right\rangle_{t}=\int_{0}^{t}\left(f^{2} h_{\bar{S}_{u}^{\circ}}^{\wp, K} \mathbf{1}_{\varphi}+f^{2} h_{\bar{S}_{u}^{K}, K}^{\sigma_{\sigma^{\prime}}}, \bar{S}_{u}^{K}\right) d u
\end{aligned}
$$

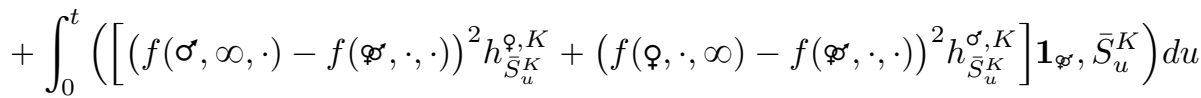

$$
\begin{aligned}
& +\int_{0}^{t}\left(\left(f(\propto, \cdot, \infty)+f\left(\sigma^{*}, \infty, \cdot\right)-f\left(\phi^{\prime}, \cdot, \cdot\right)\right)^{2} h_{\bar{S}_{u}^{K}}^{\phi, K} \mathbf{1}_{\phi}, \bar{S}_{u}^{K}\right) d u
\end{aligned}
$$

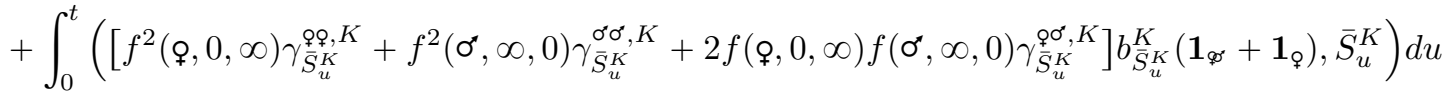

$$
\begin{aligned}
& +\int_{0}^{t}\left(\left(\left[f(\propto, \cdot, \infty)+f\left(\sigma^{\prime}, \infty, *\right)-f(ф, \cdot, *)\right]^{2} K \rho_{\bar{S}_{u}^{K}}^{K}(\bullet, *) \mathbf{1}_{q}, \bar{S}_{u}^{K}\right) . \mathbf{1}_{\sigma^{*}}, \bar{S}_{u}^{K}\right)_{*} d u .
\end{aligned}
$$

For the Central Limit Theorem, we need to include the additional parameter $\rho$ to conditions (A0)-(A4).

(A0') Same as (A0).

(A1') Same as (A1).

(A2') In addition to (A2), the same holds for $q^{K}$ being $K \rho^{K}$ and $q^{\infty}$ being $\rho^{\infty}$. 
(A3') (A3) holds for $q=b, h, m, \rho$.

(A4') Same as (A4).

Theorem 4. Under the assumptions $\left(C 0^{\prime}\right)-\left(C 3^{\prime}\right)$ and $\left(A 0^{\prime}\right)-\left(A 4^{\prime}\right)$, the process $\left(Z_{t}^{K}\right)_{t \in \mathbb{T}}$ converges weakly in $\mathbb{D}\left(\mathbb{T}, W^{-4}\right)$ as $K \rightarrow \infty$ to the process $\left(Z_{t}\right)_{t \in \mathbb{T}}$ that satisfies, for $f \in W^{4}$ and $t \in \mathbb{T}$,

$$
\begin{aligned}
& \left(f, Z_{t}\right)=\left(f, Z_{0}\right)+\int_{0}^{t}\left(\partial_{v} f+\partial_{w} f, Z_{u}\right) d u \\
& -\int_{0}^{t}\left(f \partial_{S} h_{\bar{S}_{u}, \infty}\left(Z_{u}\right) \mathbf{1}_{q}+f \partial_{S} h_{\bar{S}_{u}^{\circ, \infty}}^{\sigma_{u}}\left(Z_{u}\right) \mathbf{1}_{\sigma^{\circ},}, \bar{S}_{u}\right) d u-\int_{0}^{t}\left(f h_{\bar{S}_{u}, \infty}^{\rho} \mathbf{1}_{q}+f h_{\bar{S}_{u}, \infty}^{\sigma_{u}} \mathbf{1}_{\sigma^{\circ}}, Z_{u}\right) d u \\
& +\int_{0}^{t}\left(\left[\left(f\left(\sigma^{\prime \prime}, \infty, \cdot\right)-f\left(\Phi^{\prime}, \cdot, \cdot\right)\right) \partial_{S} h_{\bar{S}_{u}, \infty}\left(Z_{u}\right)+\left(f(\propto, \cdot, \infty)-f\left(\Phi^{\prime}, \cdot, \cdot\right)\right) \partial_{S} h_{\bar{S}_{u}, \infty}^{\sigma_{u}}\left(Z_{u}\right)\right] \mathbf{1}_{\phi^{\prime}}, \bar{S}_{u}\right) d u
\end{aligned}
$$

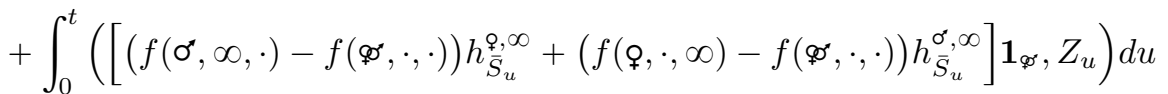

$$
\begin{aligned}
& +\int_{0}^{t}\left(\left(f(\propto, \cdot, \infty)+f\left(\sigma^{*}, \infty, \cdot\right)-f\left(\infty^{\infty}, \cdot, \cdot\right)\right) \partial_{S} h_{\bar{S}_{u}, \infty}\left(Z_{u}\right) \mathbf{1}_{\phi}, \bar{S}_{u}\right) d u
\end{aligned}
$$

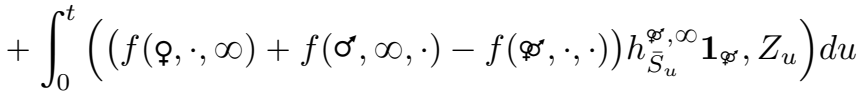

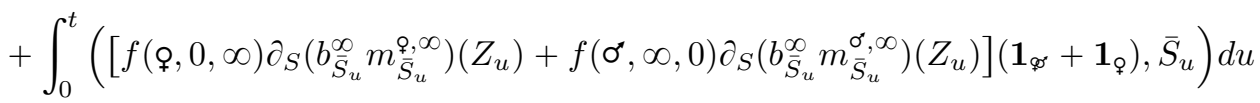

$$
\begin{aligned}
& +\int_{0}^{t}\left(\left[f(q, 0, \infty) m_{\bar{S}_{u}, \infty}+f\left(\sigma^{*}, \infty, 0\right) m_{\bar{S}_{u}, \infty}^{\sigma}\right] b_{\bar{S}_{u}}^{\infty}\left(\mathbf{1}_{\phi}+\mathbf{1}_{\varphi}\right), Z_{u}\right) d u
\end{aligned}
$$

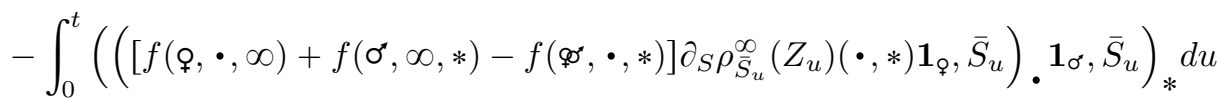

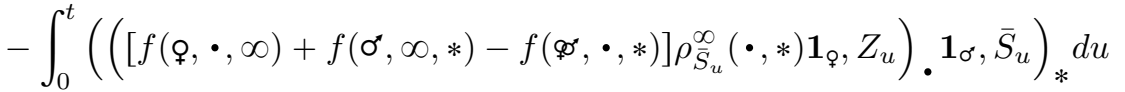

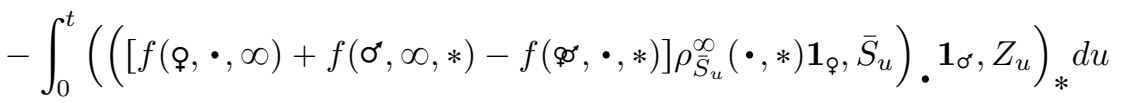

$$
\begin{aligned}
& +\tilde{M}_{t}^{f, \infty}
\end{aligned}
$$

where $\tilde{M}^{f, \infty}$ is a continuous Gaussian martingale with predictable quadratic variation

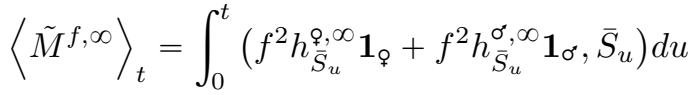

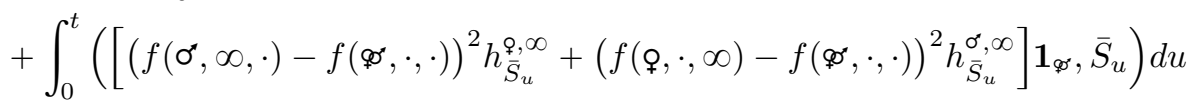

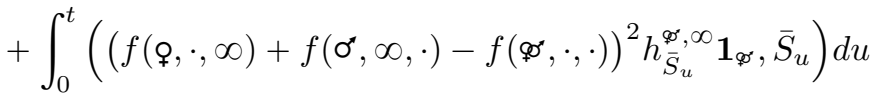

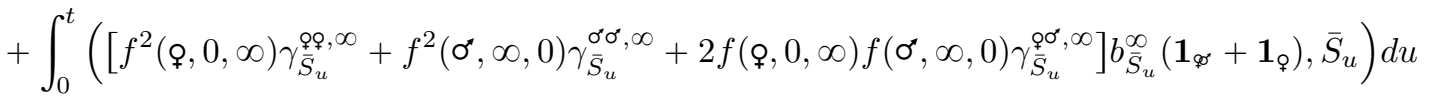

$$
\begin{aligned}
& +\int_{0}^{t}\left(\left(\left[f(\%, \cdot, \infty)+f\left(\sigma^{\prime}, \infty, *\right)-f\left(\Phi^{\prime}, \cdot, *\right)\right]^{2} \rho_{\bar{S}_{u}}^{\infty}(\cdot, *) \mathbf{1}_{\varphi}, \bar{S}_{u}\right) . \mathbf{1}_{\sigma^{*}}, \bar{S}_{u}\right)_{*} d u \text {. }
\end{aligned}
$$




\section{Appendix A. Appendices for Section 2}

Here we include the proofs of the results of this paper. We will use $c$, with or without subscript, to denote generic constants, all independent of $K$.

\section{A.1. Proofs on the model set-up.}

A.1.1. A generator formulation. A model description relying on the generator of the process was given in $\left[20\right.$. We restate it here for completeness. For $F \in C_{b}^{1}(\mathbb{R})$ and $f \in C^{1}(\mathbb{S})$, the limit

$$
\lim _{t \rightarrow 0} \frac{1}{t} \mathbb{E}_{S}\left[F\left(\left(f, S_{t}\right)\right)-F((f, S))\right]=\mathcal{G} F((f, S))
$$

exists and

$$
\begin{aligned}
\mathcal{G} F((f, S))=F^{\prime}((f, S))\left(f^{\prime}, S\right) \\
+\sum_{j=1}^{(1, S)} b_{S}\left(s_{j}\right)\left(\mathbb{E}\left[F\left(\sum_{i \in \mathbb{K}} f(i, 0) \check{\xi}_{S}^{i}\left(s_{j}\right)+(f, S)\right)\right]-F((f, S))\right) \\
+\sum_{j=1}^{(1, S)} h_{S}\left(s_{j}\right)\left(\mathbb{E}\left[F\left(\sum_{i \in \mathbb{K}} f(i, 0) \hat{\xi}_{S}^{i}\left(s_{j}\right)+(f, S)-f\left(s_{j}\right)\right)\right]-F((f, S))\right),
\end{aligned}
$$

where $(1, S)$ is the size of $S$. Consequently, Dynkin's formula holds,

$$
F\left(\left(f, S_{t}\right)\right)=F\left(\left(f, S_{0}\right)\right)+\int_{0}^{t} \mathcal{G} F\left(\left(f, S_{u}\right)\right) d u+M_{t}^{F, f},
$$

where $M_{t}^{F, f}$ is a local martingale with the predictable quadratic variation

$$
\left\langle M^{F, f}\right\rangle_{t}=\int_{0}^{t}\left(\mathcal{G} F^{2}\left(\left(f, S_{u}\right)\right)-2 F\left(\left(f, S_{u}\right)\right) \mathcal{G} F\left(\left(f, S_{u}\right)\right)\right) d u .
$$

Writing $M_{t}^{f}$ for the special case where $F$ is chosen as the identity function mapping $u$ into itself, we obtain (2).

A.1.2. A formulation through evolution. The model can also be described by analysis of the evolution of the population as done in [7 for single-type case. Recall the representation of $S_{t}$ in (11). Let $S_{t}^{(i)}(d v)=S_{t}(\{i\}, d v)=\sum_{x \in I} \mathbf{1}_{\tau_{x} \leqslant t<\sigma_{x}} \mathbf{1}_{\kappa_{x}=i} \delta_{t-\tau_{x}}(d v)$ be the age structure of the subpopulation of type $i$ individuals. Then, $\left(f, S_{t}\right)=$ $\sum_{i \in \mathbb{K}}\left(f(i, \cdot), S_{t}^{(i)}\right)$. From [7], we have, for $f \in C^{1}(\mathbb{S})$,

$$
\begin{aligned}
\left(f(i, \cdot), S_{t}^{(i)}\right)=\left(f(i, \cdot), S_{0}^{(i)}\right)+\int_{0}^{t}( & \left.f^{\prime}(i, \cdot), S_{u}^{(i)}\right) d u \\
& +f(i, 0) B^{i}([0, t])-\int_{\mathbb{A} \times[0, t]} f(i, v) D^{i}(d v, d u),
\end{aligned}
$$

where $B^{i}(t)$ is the number of individuals of type $i$ born by time $t$ and $D^{i}(v, t)$ is the number of individuals of type $i$ who died by time $t$ and whose life span was not greater than $v$ :

$$
B^{i}(t)=\sum_{x \in I} \mathbf{1}_{\kappa_{x}=i} \mathbf{1}_{\tau_{x} \leqslant t} \quad \text { and } \quad D^{i}(v, t)=\sum_{x \in I} \mathbf{1}_{\kappa_{x}=i} \mathbf{1}_{\lambda_{x} \leqslant v} \mathbf{1}_{\sigma_{x} \leqslant t} .
$$


Therefore, $S_{t}$ satisfies the following: for $f \in C^{1}(\mathbb{S})$,

$$
\left(f, S_{t}\right)=\left(f, S_{0}\right)+\int_{0}^{t}\left(f^{\prime}, S_{u}\right) d u+\sum_{i \in \mathbb{K}} f(i, 0) B^{i}([0, t])-\int_{\mathbb{S} \times[0, t]} f(s) D(d s, d u),
$$

where $D((i, v), t)=D^{i}(v, t)$. We obtain (2) by compensating the last two terms in (14).

For each $i, M_{\check{B}^{i}, f}(t):=f(i, 0)\left(\check{B}^{i}([0, t])-\int_{0}^{t}\left(b_{S_{u}} \breve{m}_{S_{u}}^{i}, S_{u}\right) d u\right)$ is a martingale with predictable quadratic variation $\left\langle M_{\check{B}^{i}, f}\right\rangle_{t}=f^{2}(i, 0) \int_{0}^{t}\left(b_{S_{u}} \check{\gamma}_{S_{u}}^{i i}, S_{u}\right) d u$, with $\breve{B}$ denoting the number of individuals born through bearings of mothers by time $t$. Sum over $i$ to obtain the compensated process

$$
M_{\breve{B}, f}(t):=\sum_{i \in \mathbb{K}} f(i, 0)\left(\breve{B}^{i}([0, t])-\int_{0}^{t}\left(b_{S_{u}} \breve{m}_{S_{u}}^{i}, S_{u}\right) d u\right)
$$

as a martingale with the predictable quadratic variation

$$
\left\langle M_{\breve{B}, f}\right\rangle_{t}=\sum_{i_{1} \in \mathbb{K}} \sum_{i_{2} \in \mathbb{K}} f\left(i_{1}, 0\right) f\left(i_{2}, 0\right) \int_{0}^{t}\left(b_{S_{u}} \breve{\gamma}_{S_{u}}^{i_{1} i_{2}}, S_{u}\right) d u
$$

Note that $\left[M_{\breve{B}^{i_{1}}, f}, M_{\check{B}^{i_{2}, f}}\right]_{t}=\sum_{u \leqslant t} \Delta M_{\check{B}^{i_{1}}, f}(u) \Delta M_{\check{B}^{i_{2}}, f}(u)$. For $i_{1} \neq i_{2}$, the product of jumps $\Delta M_{\breve{B}^{i_{1}, f}}(u) \Delta M_{\breve{B}^{i_{2}, f}}(u)$ is non-zero precisely when there is a birth of both types, in which case it is equal to

$$
\sum_{x \in I} f\left(i_{1}, 0\right) f\left(i_{2}, 0\right) \mathbf{1}_{\sigma_{x}>u}\left(\sum_{j \in \mathbb{N}} \mathbf{1}_{\tau_{x j}=u} \mathbf{1}_{\kappa_{x j}=i_{1}}\right)\left(\sum_{j \in \mathbb{N}} \mathbf{1}_{\tau_{x j}=u} \mathbf{1}_{\kappa_{x j}=i_{2}}\right) .
$$

Thus,

$$
\left\langle M_{\breve{B}^{i_{1}, f}}, M_{\breve{B}^{i_{2}, f}}\right\rangle_{t}=f\left(i_{1}, 0\right) f\left(i_{2}, 0\right) \int_{0}^{t}\left(b_{S_{u}} \breve{\gamma}_{S_{u}}^{i_{1}, i_{2}}, S_{u}\right) d u
$$

is a compensator of $\left[M_{\check{B}^{i_{1}, f}}, M_{\check{B}^{i_{2}, f}}\right]_{t}$, and, since

$$
\left\langle M_{\breve{B}, f}\right\rangle_{t}=\sum_{i_{1} \in \mathbb{K}} \sum_{i_{2} \in \mathbb{K}}\left\langle M_{\check{B}^{i_{1}}, f}, M_{\breve{B}^{i_{2}, f}}\right\rangle_{t}
$$

(15) follows. In a similar manner, with $\widehat{B}$ denoting the number of individuals generated through splitting by time $t, M_{\widehat{B}, f}(t):=\sum_{i \in \mathbb{K}} f(i, 0)\left(\widehat{B}^{i}([0, t])-\int_{0}^{t}\left(h_{S_{u}} \widehat{m}_{S_{u}}^{i}, S_{u}\right) d u\right)$ is a martingale with

$$
\left\langle M_{\widehat{B}, f}\right\rangle_{t}=\sum_{i_{1} \in \mathbb{K}} \sum_{i_{2} \in \mathbb{K}} f\left(i_{1}, 0\right) f\left(i_{2}, 0\right) \int_{0}^{t}\left(h_{S_{u}} \hat{\gamma}_{S_{u}}^{i_{1} i_{2}}, S_{u}\right) d u
$$

and $M_{D, f}(t):=\int_{\mathbb{S} \times[0, t]} f(s) D(d s, d u)-\int_{0}^{t}\left(h_{S_{u}} f, S_{u}\right) d u$ is a martingale with

$$
\left\langle M_{D, f}\right\rangle_{t}=\int_{0}^{t}\left(h_{S_{u}} f^{2}, S_{u}\right) d u .
$$

Now, let $M_{t}^{f}=M_{\breve{B}, f}(t)+M_{\widehat{B}, f}(t)-M_{D, f}(t)$. Analysing cross terms as before and adding them together, we have, since $\left\langle M_{\breve{B}, f}, M_{\widehat{B}, f}\right\rangle_{t}=0$ and $\left\langle M_{\breve{B}, f}, M_{D, f}\right\rangle_{t}=0$, 
that

$$
\begin{aligned}
& \left\langle M^{f}\right\rangle_{t}=\left\langle M_{\breve{B}, f}\right\rangle_{t}+\left\langle M_{\widehat{B}, f}\right\rangle_{t}+\left\langle M_{D, f}\right\rangle_{t}-2\left\langle M_{\widehat{B}, f}, M_{D, f}\right\rangle_{t} \\
& =\int_{0}^{t}\left(\sum_{i_{1} \in \mathbb{K}} \sum_{i_{2} \in \mathbb{K}} f\left(i_{1}, 0\right) f\left(i_{2}, 0\right) w_{S_{u}}^{i_{1} i_{2}}+h_{S_{u}} f^{2}-2 \sum_{i \in \mathbb{K}} f(i, 0) h_{S_{u}} \hat{m}_{S_{u}}^{i} f, S_{u}\right) d u .
\end{aligned}
$$

In fact, it can further be shown that, for $f, g$ on $\mathbb{S}$,

$$
\begin{aligned}
\left\langle M^{f}, M^{g}\right\rangle_{t}=\int_{0}^{t}\left(\sum_{i_{1} \in \mathbb{K}} \sum_{i_{2} \in \mathbb{K}} f\left(i_{1}, 0\right) g\left(i_{2}, 0\right) w_{S_{u}}^{i_{1} i_{2}}+h_{S_{u}} f g\right. \\
\\
\left.\quad-\sum_{i \in \mathbb{K}}(f(i, 0) g+g(i, 0) f) h_{S_{u}} \hat{m}_{S_{u}}^{i}, S_{u}\right) d u .
\end{aligned}
$$

A.1.3. Proof of Remark 3, Let

$$
\begin{aligned}
M_{t}(d s)= & \sum_{i \in \mathbb{K}} \delta_{(i, 0)}(d s)\left(\breve{B}^{i}([0, t])-\int_{0}^{t}\left(b_{S_{u}} \check{m}_{S_{u}}^{i}, S_{u}\right) d u\right) \\
& +\sum_{i \in \mathbb{K}} \delta_{(i, 0)}(d s)\left(\widehat{B}^{i}([0, t])-\int_{0}^{t}\left(h_{S_{u}} \widehat{m}_{S_{u}}^{i}, S_{u}\right) d u\right) \\
- & \left(\sum_{x \in I} \delta_{\left(\kappa_{x}, \lambda_{x}\right)}(d s) \mathbf{1}_{\sigma_{x} \leqslant t}-\int_{0}^{t} \sum_{x \in I} \delta_{\left(\kappa_{x}, u-\tau_{x}\right)}(d s) h_{S_{u}}(s) \mathbf{1}_{\tau_{x} \leqslant u<\sigma_{x}} d u\right),
\end{aligned}
$$

then $\left(f, M_{t}\right)=M_{t}^{f}$. For $g \in C^{0}(\mathbb{S})$ and $\varphi \in C^{0}(\mathbb{T}), \int_{0}^{t} \varphi(u) d\left(g, M_{u}\right)$ is a martingale with

$$
\begin{aligned}
\left\langle\int_{0} \varphi(u) d\left(g, M_{u}\right)\right\rangle_{t}= & \int_{0}^{t} \varphi^{2}(u)\left(\sum_{i_{1} \in \mathbb{K}} \sum_{i_{2} \in \mathbb{K}} g\left(i_{1}, 0\right) g\left(i_{2}, 0\right) w_{S_{u}}^{i_{1} i_{2}}\right. \\
& \left.\quad+h_{S_{u}} g^{2}(\cdot, u)-2 \sum_{i \in \mathbb{K}} g(i, 0, u) h_{S_{u}} \hat{m}_{S_{u}}^{i} g(\cdot, u), S_{u}\right) d u .
\end{aligned}
$$

Write $\int_{0}^{t}\left(\varphi(u) g, d M_{u}\right)$ for $\int_{0}^{t} \varphi(u) d\left(g, M_{u}\right)$. Then, by the Monotone Class Theorem (see e.g. [5, I.22.1]), $\int_{0}^{t}\left(f(\cdot, u), d M_{u}\right)$ is a martingale for any $f \in C^{0}(\mathbb{S} \times \mathbb{T})$ whose predictable quadratic variation coincides with (3).

A.2. Proof of the Law of Large Numbers. This is done by checking the tightness of the scaled sequence $\bar{S}^{K}$ and the uniqueness of the limiting process. By Jakubowski's theorem [21], $\left\{\bar{S}^{K}\right\}$ is tight in $\mathbb{D}(\mathbb{T}, \mathcal{M})$ if:

(J1) For each $\eta>0$, there exists a compact set $\mathcal{C}_{\eta} \in \mathcal{M}$ such that

$$
\liminf _{K \rightarrow \infty} \mathbb{P}\left(\bar{S}_{t}^{K} \in \mathcal{C}_{\eta} \forall t \in \mathbb{T}\right)>1-\eta .
$$

(J2) For each $f \in C^{1},\left\{\left(f, \bar{S}^{K}\right)\right\}$ is tight in $\mathbb{D}(\mathbb{T}, \mathbb{R})$.

In terms of the semimartingale decomposition $\left(f, \bar{S}_{t}^{K}\right)=V_{t}^{f, K}+\bar{M}_{t}^{f, K},(\mathrm{~J} 2)$ reduces to the following Aldous-Rebolledo criteria:

(J2a) For each $t \in \mathbb{T},\left(f, \bar{S}_{t}^{K}\right)$ is tight, that is, for each $\epsilon>0$, there exist $\delta>0$ such that for all $K, \mathbb{P}\left(\left|\left(f, \bar{S}_{t}^{K}\right)\right|>\delta\right)<\epsilon$. 
(J2b) For each $\epsilon_{1}, \epsilon_{2}>0$, there exist $\delta>0$ and $K_{0} \geqslant 1$ such that for every sequence of stopping times $\tau^{K} \leqslant T$,

$$
\begin{gathered}
\sup _{K>K_{0}} \sup _{\zeta<\delta} \mathbb{P}\left(\left|V_{\tau^{K}+\zeta \wedge T}^{f, K}-V_{\tau^{K}}^{f, K}\right|>\epsilon_{1}\right)<\epsilon_{2}, \\
\sup _{K>K_{0}} \sup _{\zeta<\delta} \mathbb{P}\left(\left|\left\langle\bar{M}^{f, K}\right\rangle_{\tau^{K}+\zeta \wedge T}-\left\langle\bar{M}^{f, K}\right\rangle_{\tau^{K}}\right|>\epsilon_{1}\right)<\epsilon_{2} .
\end{gathered}
$$

A.2.1. Preliminary estimates. Recall the operator $L_{S}^{K}$ defined in (5). Let $\hat{L}_{S}^{K}$ be such that

$$
\widehat{L}_{S}^{K} f=-h_{S}^{K} f+\sum_{i \in \mathbb{K}} f(i, 0) n_{S}^{i, K},
$$

so that $L_{S}^{K} f=f^{\prime}+\widehat{L}_{S}^{K} f$. Define also the operator $\Pi_{S}^{K}$ such that

$$
\Pi_{S}^{K} f=\sum_{i_{1} \in \mathbb{K}} \sum_{i_{2} \in \mathbb{K}} f\left(i_{1}, 0\right) f\left(i_{2}, 0\right) w_{S}^{i_{1} i_{2}, K}+h_{S}^{K} f^{2}-2 \sum_{i \in \mathbb{K}} f(i, 0) h_{S}^{K} \hat{m}_{S}^{i, K} f,
$$

so that $\left\langle M^{f, K}\right\rangle_{t}=\int_{0}^{t}\left(\Pi_{\bar{S}_{u}^{K}}^{K} f, S_{u}^{K}\right) d u$.

Proposition 2. Suppose (CO) holds. Then, for any $f \in C^{0}$,

$$
\left|\Pi_{S}^{K} f\right| \leqslant c\|f\|_{C^{0}}^{2} \quad \text { and } \quad\left|\widehat{L}_{S}^{K} f\right| \leqslant c\|f\|_{C^{0}}
$$

and for any $f \in C^{1}$,

$$
\left|L_{S}^{K} f\right| \leqslant c|| f \|_{C^{1}}
$$

In particular, $\left|\Pi_{S}^{K} 1\right| \leqslant c$ and $\left|L_{S}^{K} 1\right|=\left|\widehat{L}_{S}^{K} 1\right| \leqslant c$.

Proof. This follows immediately from the boundedness of the parameters and the definition of $\|f\|_{C^{j}}$.

Proposition 3. Suppose (CO) and (C3) hold. Then,

$$
\mathbb{E}\left[\left(1, \bar{S}_{t}^{K}\right)\right] \leqslant\left(1, \bar{S}_{0}^{K}\right) e^{c t}
$$

and

$$
\sup _{K \geqslant 1} \mathbb{E}\left[\sup _{t \leqslant T}\left(1, \bar{S}_{t}^{K}\right)\right]<\infty .
$$

Proof. From (4) and Proposition 2,

$$
\left(1, \bar{S}_{t}^{K}\right) \leqslant\left(1, \bar{S}_{0}^{K}\right)+c_{1} \int_{0}^{t}\left(1, \bar{S}_{u}^{K}\right) d u+\frac{1}{K} M_{t}^{1, K}
$$

Taking expectation and applying Gronwall's inequality, we establish the first statement, $\mathbb{E}\left[\left(1, \bar{S}_{t}^{K}\right)\right] \leqslant\left(1, \bar{S}_{0}^{K}\right) e^{c_{1} t}$.

For the second statement, note that

$$
\sup _{t \leqslant T}\left(1, \bar{S}_{t}^{K}\right) \leqslant\left(1, \bar{S}_{0}^{K}\right)+c_{1} \int_{0}^{T}\left(1, \bar{S}_{u}^{K}\right) d u+\frac{1}{K} \sup _{t \leqslant T} M_{t}^{1, K} .
$$

Taking expectation and using the first statement,

$$
\mathbb{E}\left[\sup _{t \leqslant T}\left(1, \bar{S}_{t}^{K}\right)\right] \leqslant\left(1, \bar{S}_{0}^{K}\right)+c_{1}\left(1, \bar{S}_{0}^{K}\right) e^{c_{1} T} T+\frac{1}{K} \mathbb{E}\left[\sup _{t \leqslant T} M_{t}^{1, K}\right] .
$$

Now,

$$
\mathbb{E}\left[\sup _{t \leqslant T} M_{t}^{1, K}\right]^{2} \leqslant \mathbb{E}\left[\sup _{t \leqslant T}\left(M_{t}^{1, K}\right)^{2}\right] \leqslant 4 \mathbb{E}\left[\left\langle M^{1, K}\right\rangle_{T}\right]
$$


by Doob's inequality; using Proposition 2 and the first statement again,

$$
\mathbb{E}\left[\left\langle M^{1, K}\right\rangle_{T}\right] \leqslant c_{2} \int_{0}^{T} \mathbb{E}\left[\left(1, S_{u}^{K}\right)\right] d u \leqslant c_{2} K\left(1, \bar{S}_{0}^{K}\right) e^{c_{1} T} T .
$$

Therefore,

$$
\mathbb{E}\left[\sup _{t \leqslant T}\left(1, \bar{S}_{t}^{K}\right)\right] \leqslant\left(1, \bar{S}_{0}^{K}\right)\left(1+c_{1} e^{c_{1} T} T\right)+\frac{1}{\sqrt{K}} c_{3}\left(1, \bar{S}_{0}^{K}\right)^{1 / 2} e^{c_{4} T} T^{1 / 2},
$$

which is bounded in $K$ by (C3).

A.2.2. Tightness of $\bar{S}^{K}$.

Proposition 4. Suppose (CO) and (C3) hold. Then, the sequence $\bar{S}^{K}$ is tight in $\mathbb{D}(\mathbb{T}, \mathcal{M})$.

Proof. First, we show that (J1) holds. From Markov's inequality and (18) we have

$$
\mathbb{P}\left(\sup _{t \leqslant T}\left(1, \bar{S}_{t}^{K}\right)>\delta\right) \leqslant \frac{c}{\delta} .
$$

This ensures the existence of $\delta_{\eta}$ such that $\mathbb{P}\left(\sup _{t \leqslant T}\left(1, \bar{S}_{t}^{K}\right)>\delta_{\eta}\right) \leqslant \eta$. Let

$$
\mathcal{C}(\delta)=\{\mu \in \mathcal{M}:(1, \mu) \leqslant \delta\} .
$$

Then $\mathcal{C}(\delta)$ is compact and for any $\eta>0$,

$$
\liminf _{K \rightarrow \infty} \mathbb{P}\left(\bar{S}_{t}^{K} \in \mathcal{C}\left(\delta_{\eta}\right) \forall t \in \mathbb{T}\right)>1-\eta .
$$

Next, we show that (J2a) and (J2b) hold. Condition (J2a) is immediate from Markov's inequality

$$
\mathbb{P}\left(\left|\left(f, \bar{S}_{t}^{K}\right)\right|>\delta\right) \leqslant \frac{1}{\delta} \mathbb{E}\left[\left|\left(f, \bar{S}_{t}^{K}\right)\right|\right] \leqslant \frac{1}{\delta}\|f\|_{C^{1}} \mathbb{E}\left[\left(1, \bar{S}_{t}^{K}\right)\right]
$$

and by using (17).

For $(\mathrm{J} 2 \mathrm{~b})(\mathrm{i})$, note that, using Proposition 2 ,

$$
\begin{aligned}
\left|V_{\tau^{K}+\zeta \wedge T}^{f, K}-V_{\tau^{K}}^{f, K}\right| & \leqslant c_{1}\|f\|_{C^{1}} \int_{\tau^{K}}^{\tau^{K}+\zeta \wedge T}\left(1, \bar{S}_{u}^{K}\right) d u \\
& =c_{1}\|f\|_{C^{1}} \int_{0}^{\zeta}\left(1, \bar{S}_{\tau^{K}+u \wedge T}^{K}\right) d u \leqslant c_{1}\|f\|_{C^{1}} \delta \sup _{t \leqslant T}\left(1, \bar{S}_{t}^{K}\right)
\end{aligned}
$$

and thus

$$
\mathbb{E}\left[\left|V_{\tau^{K}+\zeta \wedge T}^{f, K}-V_{\tau^{K}}^{f, K}\right|\right] \leqslant c_{1}\|f\|_{C^{1}} \delta \mathbb{E}\left[\sup _{t \leqslant T}\left(1, \bar{S}_{t}^{K}\right)\right]
$$

is bounded by (18). Therefore, with Markov's inequality, we can choose $\delta$ such that (i) holds.

For $(\mathrm{J} 2 \mathrm{~b})(\mathrm{ii})$, by Proposition 2

$$
\begin{aligned}
\mid\left\langle\bar{M}^{f, K}\right\rangle_{\tau^{K}+\zeta \wedge T} & -\left\langle\bar{M}^{f, K}\right\rangle_{\tau^{K}} \mid \leqslant \frac{1}{K^{2}} \int_{\tau^{K}}^{\tau^{K}+\zeta \wedge T}\left(\left|\Pi_{\bar{S}_{u}^{K}}^{K} f\right|, S_{u}^{K}\right) d u \\
& \leqslant c_{2}\|f\|_{C^{1}}^{2} \frac{1}{K^{2}} \int_{\tau^{K}}^{\tau^{K}+\zeta \wedge T}\left(1, S_{u}^{K}\right) d u \leqslant c_{2}|| f \|_{C^{1}}^{2} \delta \frac{1}{K^{2}} \sup _{t \leqslant T}\left(1, S_{t}^{K}\right)
\end{aligned}
$$

using a similar argument as for (i). Thus,

$$
\mathbb{E}\left[\left|\left\langle\bar{M}^{f, K}\right\rangle_{\tau^{K}+\zeta \wedge T}-\left\langle\bar{M}^{f, K}\right\rangle_{\tau^{K}}\right|\right] \leqslant c_{2}\|f\|_{C^{1}}^{2} \delta \frac{1}{K} \mathbb{E}\left[\sup _{t \leqslant T}\left(1, \bar{S}_{t}^{K}\right)\right] .
$$


The proof is then complete by (18) and Markov's inequality.

Remark 4. From the proof of Proposition 4, we can see that the martingale $\bar{M}^{f, K}=\frac{1}{K} M^{f, K}$ is tight for any $f \in C^{1}$. Moreover, it converges to 0 as $K$ tends to infinity, since the predictable quadratic variation vanishes.

A.2.3. Convergence of $\bar{S}^{K}$ and the limiting process. Tightness implies the existence of a subsequence that converges. We now identify the limit of $\bar{S}^{K}$ and show the uniqueness of the limiting process.

Proposition 5. Suppose (CO) - (C3) hold. Every limit point $\mathcal{S}$ of the sequence $\bar{S}^{K}$ satisfies the equation

$$
\left(f, \mathcal{S}_{t}\right)=\left(f, \mathcal{S}_{0}\right)+\int_{0}^{t}\left(L_{\mathcal{S}_{u}}^{\infty} f, \mathcal{S}_{u}\right) d u
$$

for any $f \in C^{1}$ and $t \in \mathbb{T}$, where $L_{S}^{\infty} f$ is as defined in (7).

Proof. From Remark 4, the martingale sequence $\bar{M}^{f, K}$ vanishes as $K$ tends to infinity. This together with the convergence of $\bar{S}_{0}^{K}$ by (C3), it remains to show the convergence of $\int_{0}^{t}\left(L_{\bar{S}_{u}^{K}}^{K} f, \bar{S}_{u}^{K}\right) d u$ to $\int_{0}^{t}\left(L_{\mathcal{S}_{u}}^{\infty} f, \mathcal{S}_{u}\right) d u$. Note that, by $(\mathrm{C} 1)$ and $(\mathrm{C} 2)$,

$$
\left\|h_{\bar{S}_{u}^{K}}^{K}-h_{\mathcal{S}_{u}}^{\infty}\right\|_{\infty} \leqslant\left\|h_{\bar{S}_{u}^{K}}^{K}-h_{\mathcal{S}_{u}}^{K}\right\|_{\infty}+\left\|h_{\mathcal{S}_{u}}^{K}-h_{\mathcal{S}_{u}}^{\infty}\right\|_{\infty} \rightarrow 0 ;
$$

similarly for other model parameters. Thus, $\left\|L_{\bar{S}_{u}^{K}}^{K} f-L_{\mathcal{S}_{u}}^{\infty} f\right\|_{\infty} \rightarrow 0$ and

$$
\int_{0}^{t}\left|\left(L_{\bar{S}_{u}^{K}}^{K} f-L_{\mathcal{S}_{u}}^{\infty} f, \bar{S}_{u}^{K}\right)\right| d u \leqslant \int_{0}^{t}\left\|L_{\bar{S}_{u}^{K}}^{K} f-L_{\mathcal{S}_{u}}^{\infty} f\right\|_{\infty}\left(1, \bar{S}_{u}^{K}\right) d u \rightarrow 0
$$

by dominated convergence theorem. Note also that, for any $f \in C^{1}$,

$$
\left|L_{S}^{\infty} f\right| \leqslant\left\|f^{\prime}\right\|_{\infty}+c_{1}\|f\|_{\infty} \leqslant c_{2}\|f\|_{C^{1}} .
$$

Thus,

$$
\int_{0}^{t}\left|\left(L_{\mathcal{S}_{u}}^{\infty} f, \bar{S}_{u}^{K}-\mathcal{S}_{u}\right)\right| d u \leqslant \int_{0}^{t}\left\|L_{\mathcal{S}_{u}}^{\infty} f\right\|_{\infty}\left\|\bar{S}_{u}^{K}-\mathcal{S}_{u}\right\| d u \leqslant c_{2}\|f\|_{C^{1}} \int_{0}^{t}\left\|\bar{S}_{u}^{K}-\mathcal{S}_{u}\right\| d u
$$

vanishes as $K \rightarrow \infty$. Hence,

$$
\begin{aligned}
\mid \int_{0}^{t}\left(L_{\bar{S}_{u}^{K}}^{K} f, \bar{S}_{u}^{K}\right) d u-\int_{0}^{t}( & \left(L_{\mathcal{S}_{u}}^{\infty} f, \mathcal{S}_{u}\right) d u \mid \\
& \leqslant \int_{0}^{t}\left(\left|\left(L_{\bar{S}_{u}^{K}}^{K} f-L_{\mathcal{S}_{u}}^{\infty} f, \bar{S}_{u}^{K}\right)\right|+\left|\left(L_{\mathcal{S}_{u}}^{\infty} f, \bar{S}_{u}^{K}-\mathcal{S}_{u}\right)\right|\right) d u
\end{aligned}
$$

converges to zero.

It remains to show the uniqueness of the solution to (19). To do this, we introduce an alternative representation to (19).

Proposition 6. For $\phi \in C^{1}$, define the shift operator $\hat{\Theta}_{r}$ such that for each $r \in \mathbb{T}$ and $s=(i, v), v \in[0, \omega-r], \widehat{\Theta}_{r} \phi(s)=\widehat{\Theta}_{r} \phi(i, v)=\phi(i, v+r)$ and $\widehat{\Theta}_{r} \phi \in C^{0}$ with $\left\|\hat{\Theta}_{r} \phi\right\|_{C^{0}} \leqslant c\|\phi\|_{C^{0}}$. (This is possible by reflecting $\phi$ about $v=\omega-r$, in which case $\left\|\hat{\Theta}_{r} \phi\right\|_{C^{0}} \leqslant\|\phi\|_{C^{0}}$.) Then (19) is equivalent to the following: for $\phi \in C^{1}$,

$$
\left(\phi, \mathcal{S}_{t}\right)=\left(\hat{\Theta}_{t} \phi, \mathcal{S}_{0}\right)+\int_{0}^{t}\left(-h_{\mathcal{S}_{u}}^{\infty} \widehat{\Theta}_{t-u} \phi+\sum_{i \in \mathbb{K}} \widehat{\Theta}_{t-u} \phi(i, 0) n_{\mathcal{S}_{u}}^{i, \infty}, \mathcal{S}_{u}\right) d u .
$$


Proof. Let $g(s, t)=f(s) \varphi(t)$, where $f$ and $\varphi$ are functions in $C^{1}(\mathbb{T})$ and $C^{1}(\mathbb{S})$ respectively. Then,

$$
\begin{aligned}
\left(g(\cdot, t), \mathcal{S}_{t}\right) & =\varphi(0)\left(f, \mathcal{S}_{0}\right)+\int_{0}^{t} \varphi^{\prime}(u)\left(f, \mathcal{S}_{u}\right) d u+\int_{0}^{t} \varphi(u)\left(L_{\mathcal{S}_{u}}^{\infty} f, \mathcal{S}_{u}\right) d u \\
& =\left(g(\cdot, 0), \mathcal{S}_{0}\right)+\int_{0}^{t}\left(\partial_{1} g(\cdot, u)+\partial_{2} g(\cdot, u)-h_{\mathcal{S}_{u}}^{\infty} g(\cdot, u)+\sum_{i \in \mathbb{K}} g(i, 0, u) n_{\mathcal{S}_{u}}^{i, \infty}, \mathcal{S}_{u}\right) d u .
\end{aligned}
$$

By the Monotone Class Theorem (e.g. [5. I.22.1]), this holds for any $g \in C^{1,1}(\mathbb{S} \times \mathbb{T})$. Now, fix $t \in \mathbb{T}$ and $\phi \in C^{1}(\mathbb{S})$, take $g(s, u)=\widehat{\Theta}_{t-u} \phi(s)=\phi(i, v+t-u)$ for $s \in \mathbb{S}$ and $u \in[0, t]$, then we obtain (20). From (20), we can also recover (19) by noting that $\widehat{\Theta}_{t-u} \phi(s)=\phi(s)+\int_{u}^{t} \widehat{\Theta}_{w-u} \phi^{\prime}(s) d w$ and applying Fubini's theorem.

Now, we can show the uniqueness of the solution to (19) by showing the uniqueness of the solution to (20).

Proposition 7. If $\mathcal{S}^{1}$ and $\mathcal{S}^{2}$ both satisfy (20) with $\mathcal{S}_{0}^{1}=\mathcal{S}_{0}^{2}$, then $\mathcal{S}^{1}=\mathcal{S}^{2}$.

Proof. Let

$$
\widehat{L}_{S}^{\infty} f=-h_{S}^{\infty} f+\sum_{i \in \mathbb{K}} f(i, 0) n_{S}^{i, \infty}
$$

so that (20) becomes $\left(\phi, \mathcal{S}_{t}\right)=\left(\widehat{\Theta}_{t} \phi, \mathcal{S}_{0}\right)+\int_{0}^{t}\left(\widehat{L}_{\mathcal{S}_{u}}^{\infty} \widehat{\Theta}_{t-u} \phi, \mathcal{S}_{u}\right) d u$. We have, for $\phi \in C^{1}$,

$$
\begin{aligned}
\left|\left(\phi, \mathcal{S}_{t}^{1}-\mathcal{S}_{t}^{2}\right)\right| & \leqslant \int_{0}^{t}\left|\left(\widehat{L}_{\mathcal{S}_{u}^{1}}^{\infty} \hat{\Theta}_{t-u} \phi-\widehat{L}_{\mathcal{S}_{u}^{2}}^{\infty} \widehat{\Theta}_{t-u} \phi, \mathcal{S}_{u}^{1}\right)\right|+\left|\left(\widehat{L}_{\mathcal{S}_{u}^{2}}^{\infty} \widehat{\Theta}_{t-u} \phi, \mathcal{S}_{u}^{1}-\mathcal{S}_{u}^{2}\right)\right| d u \\
& \leqslant \int_{0}^{t}\left\|\widehat{L}_{\mathcal{S}_{u}^{1}}^{\infty} \widehat{\Theta}_{t-u} \phi-\widehat{L}_{\mathcal{S}_{u}^{2}}^{\infty} \widehat{\Theta}_{t-u} \phi\right\|_{\infty}\left(1, \mathcal{S}_{u}^{1}\right)+\left\|\widehat{L}_{\mathcal{S}_{u}^{2}}^{\infty} \widehat{\Theta}_{t-u} \phi\right\|\left\|_{\infty}\right\| \mathcal{S}_{u}^{1}-\mathcal{S}_{u}^{2} \| d u .
\end{aligned}
$$

Now, by $(\mathrm{C} 1)$,

$$
\begin{aligned}
\left\|h_{\mu}^{\infty}-h_{\nu}^{\infty}\right\|_{\infty} & \leqslant\left\|h_{\mu}^{\infty}-h_{\mu}^{K}\right\|_{\infty}+\left\|h_{\mu}^{K}-h_{\nu}^{K}\right\|_{\infty}+\left\|h_{\nu}^{K}-h_{\nu}^{\infty}\right\|_{\infty} \\
& \leqslant\left\|h_{\mu}^{\infty}-h_{\mu}^{K}\right\|_{\infty}+c\|\mu-\nu\|+\left\|h_{\nu}^{K}-h_{\nu}^{\infty}\right\|_{\infty} ;
\end{aligned}
$$

taking the limit $K \rightarrow \infty$ on both sides, we obtain $\left\|h_{\mu}^{\infty}-h_{\nu}^{\infty}\right\|_{\infty} \leqslant c_{1}\|\mu-\nu\|$. Similarly for other model parameters. Thus, $\left\|\widehat{L}_{\mu}^{\infty} f-\widehat{L}_{\nu}^{\infty} f\right\|_{\infty} \leqslant c_{2}\|f\|_{\infty}\|\mu-\nu\|$. We also have by $(\mathrm{C} 0)$ and $(\mathrm{C} 2)$ that $\left\|\widehat{L}_{\mu}^{\infty} f\right\|_{\infty} \leqslant c_{3}\|f\|_{\infty}$. Finally, since also $\left\|\widehat{\Theta}_{r} \phi\right\|_{C^{0}} \leqslant$ $c\|\phi\|_{C^{0}}$ for any $r \in \mathbb{T}$, we have

$$
\left|\left(\phi, \mathcal{S}_{t}^{1}-\mathcal{S}_{t}^{2}\right)\right| \leqslant \int_{0}^{t}\left(c_{4}\|\phi\|_{C^{0}}\left\|\mathcal{S}_{u}^{1}-\mathcal{S}_{u}^{2}\right\|\left(1, \mathcal{S}_{u}^{1}\right)+c_{5}\|\phi\|_{C^{0}}\left\|\mathcal{S}_{u}^{1}-\mathcal{S}_{u}^{2}\right\|\right) d u
$$

Using $(\mathrm{C} 0)$ and Gronwall's inequality, we can show that $\left(1, \mathcal{S}_{u}^{1}\right) \leqslant\left(1, \mathcal{S}_{0}^{1}\right) e^{c_{6} u}$. Therefore, it follows that

$$
\left|\left(\phi, \mathcal{S}_{t}^{1}-\mathcal{S}_{t}^{2}\right)\right| \leqslant c_{7}\|\phi\|_{C^{0}}\left(1+\left(1, \mathcal{S}_{0}^{1}\right) e^{c_{6} T}\right) \int_{0}^{t}\left\|\mathcal{S}_{u}^{1}-\mathcal{S}_{u}^{2}\right\| d u .
$$

Note that for any $\phi \in C^{0}$, there exists a sequence $\left\{\phi_{n}\right\}$ such that $\phi_{n} \in C^{1}$ and $\phi_{n}$ converges uniformly to $\phi$. Thus, (21) holds for any $\phi \in C^{0}$, and $\left\|\mathcal{S}_{t}^{1}-\mathcal{S}_{t}^{2}\right\|=0$ by Gronwall's inequality. 1 .

Putting all together, we established the convergence of $\bar{S}^{K}$ as stated in Theorem 
A.3. Proof of the Central Limit Theorem. This is in line with the single-type case [7] and starts from an alternative representation of $Z_{t}^{K}$, which is a similar trick as in Proposition 6.

\section{A.3.1. Alternative representation.}

Remark 5. Like in [7, we take $\Theta_{r} \phi$ as the function on $\mathbb{S}$ such that for each $r \in \mathbb{T}$ and $s=(i, v), v \in[0, \omega-r], \Theta_{r} \phi(s)=\Theta_{r} \phi(i, v)=\phi(i, v+r)$ and, whenever $\phi \in W^{j}$, then $\Theta_{r} \phi \in W^{j}$ with

$$
\left\|\Theta_{r} \phi\right\|_{W^{j}} \leqslant c\|\phi\|_{W^{j}}
$$

The existence of such a function was proved in [7].

Equation (8) can also be extended to test functions dependent on $t$, that is, for $f \in C^{1,1}(\mathbb{S} \times \mathbb{T})$. Then, for fix $t$, by taking $f(s, u)=\Theta_{t-u} \phi(s)$ for $u \leqslant t$, we have the following equation.

Proposition 8. For $\phi \in C^{1}$ and $t \in \mathbb{T}$,

$$
\begin{aligned}
\left(\phi, Z_{t}^{K}\right) & =\left(\Theta_{t} \phi, Z_{0}^{K}\right) \\
+ & \sqrt{K} \int_{0}^{t}\left(-\left(h_{\bar{S}_{u}^{K}}^{K}-h_{\bar{S}_{u}}^{\infty}\right) \Theta_{t-u} \phi+\sum_{i \in \mathbb{K}} \Theta_{t-u} \phi(i, 0)\left(n_{\bar{S}_{u}^{K}}^{i, K}-n_{\bar{S}_{u}}^{i, \infty}\right), \bar{S}_{u}\right) d u \\
+ & \int_{0}^{t}\left(-h_{\bar{S}_{u}^{K}}^{K} \Theta_{t-u} \phi+\sum_{i \in \mathbb{K}} \Theta_{t-u} \phi(i, 0) n_{\bar{S}_{u}^{K}}^{i, K}, Z_{u}^{K}\right) d u+\int_{0}^{t}\left(\Theta_{t-u} \phi, d \tilde{M}_{u}^{K}\right),
\end{aligned}
$$

where $\tilde{M}=\frac{1}{\sqrt{K}} M$ and $M$ is the measure as defined in Remark 3 .

Proof. Let $f \in C^{1}(\mathbb{S})$ and $\varphi \in C^{1}(\mathbb{T})$. For $g: \mathbb{S} \times \mathbb{T} \rightarrow \mathbb{R}$ such that $g(s, t)=\varphi(t) f(s)$,

$$
\begin{aligned}
\left(g(\cdot, t), Z_{t}^{K}\right) & =\left(\varphi(0) f, Z_{0}^{K}\right)+\int_{0}^{t} \varphi^{\prime}(u)\left(f, Z_{u}^{K}\right) d u \\
& +\sqrt{K} \int_{0}^{t} \varphi(u)\left(-\left(h_{S_{u}^{K}}^{K}-h_{\bar{S}_{u}}^{\infty}\right) f+\sum_{i=1}^{k} f(i, 0)\left(n_{S_{u}^{K}}^{i, K}-n \bar{S}_{u}^{i, \infty}\right), \bar{S}_{u}\right) d u \\
& +\int_{0}^{t} \varphi(u)\left(f^{\prime}-h_{S_{u}^{K}}^{K} f+\sum_{i=1}^{k} f(i, 0) n_{S_{u}^{K}}^{i, K}, Z_{u}^{K}\right) d u+\int_{0}^{t} \varphi(u) d\left(f, \tilde{M}_{u}^{K}\right) .
\end{aligned}
$$

We shall write $\int_{0}^{t}\left(\varphi(u) f, d \tilde{M}_{u}^{K}\right)$ for $\int_{0}^{t} \varphi(u) d\left(f, \tilde{M}_{u}^{K}\right)$. Then, we have

$$
\begin{aligned}
(g(\cdot, t) & \left., Z_{t}^{K}\right)=\left(g(\cdot, 0), Z_{0}^{K}\right) \\
& +\sqrt{K} \int_{0}^{t}\left(-\left(h_{S_{u}^{K}}^{K}-h_{\bar{S}_{u}}^{\infty}\right) g(\cdot, u)+\sum_{i=1}^{k} g((i, 0), u)\left(n_{S_{u}^{K}}^{i, K}-n_{\bar{S}_{u}}^{i, \infty}\right), \bar{S}_{u}\right) d u \\
& +\int_{0}^{t}\left(\partial_{1} g(\cdot, u)+\partial_{2} g(\cdot, u)-h_{S_{u}^{K}}^{K} g(\cdot, u)+\sum_{i=1}^{k} g((i, 0), u) n_{S_{u}^{K}}^{i, K}, Z_{u}^{K}\right) d u \\
& +\int_{0}^{t}\left(g(\cdot, u), d \tilde{M}_{u}^{K}\right) .
\end{aligned}
$$

By the Monotone Class Theorem (e.g. [5, I.22.1]), (24) holds for $g \in C^{1,1}(\mathbb{S} \times \mathbb{T})$. Now, taking $g(s, u)=\Theta_{t-u} \phi(s)=\Theta_{t-u} \phi(i, v):=\phi(i, v+t-u)$, we can conclude (23). 
A.3.2. Preliminary estimates. Recall the operator $\widehat{L}_{S}^{K}$ defined in (16) and its limit $\widehat{L}_{S}^{\infty}$.

Proposition 9. Suppose (A2) and (A3) hold. Then, for any $f \in W^{j}, j \in \mathbb{N}$, and $t \in \mathbb{T}$,

$$
\left\|\sqrt{K}\left(\widehat{L}_{\bar{S}_{t}^{K}}^{K}-\widehat{L}_{\bar{S}_{t}}^{\infty}\right) f\right\|_{\infty} \leqslant c\left(1+\left\|Z_{t}^{K}\right\|_{W^{-4}}\right)\|f\|_{W^{j}} .
$$

Proof. By the triangle inequality,

$$
\begin{gathered}
\left\|h_{\bar{S}_{t}^{K}}^{K}-h_{\bar{S}_{t}}^{\infty}\right\|_{\infty} \leqslant\left\|h_{\bar{S}_{t}^{K}}^{K}-h_{\bar{S}_{t}^{K}}^{\infty}\right\|_{\infty}+\left\|h_{\bar{S}_{t}^{K}}^{\infty}-h_{\bar{S}_{t}}^{\infty}-\partial_{S} h_{\bar{S}_{t}}^{\infty}\left(\bar{S}_{t}^{K}-\bar{S}_{t}\right)\right\|_{\infty} \\
+\left\|\partial_{S} h_{\bar{S}_{t}}^{\infty}\right\|_{\mathbb{L}^{-4}}\left\|\bar{S}_{t}^{K}-\bar{S}_{t}\right\|_{W^{-4}}
\end{gathered}
$$

and thus,

$$
\begin{aligned}
\sqrt{K} \| h_{\bar{S}_{t}^{K}}^{K}- & h_{\bar{S}_{t}}^{\infty}\left\|_{\infty} \leqslant \sqrt{K}\right\| h_{\bar{S}_{t}^{K}}^{K}-h_{\bar{S}_{t}^{K}}^{\infty} \|_{\infty} \\
& +\frac{\left\|Z_{t}^{K}\right\|_{W^{-4}}}{\left\|\bar{S}_{t}^{K}-\bar{S}_{t}\right\|_{W^{-4}}}\left\|h_{\bar{S}_{t}^{K}}^{\infty}-h_{\bar{S}_{t}}^{\infty}-\partial_{S} h_{\bar{S}_{t}}^{\infty}\left(\bar{S}_{t}^{K}-\bar{S}_{t}\right)\right\|_{\infty}+c_{1}\left\|Z_{t}^{K}\right\|_{W^{-4}},
\end{aligned}
$$

where the bound in the last term is due to (A3). The first term is bounded by (A2) and the second term is bounded by (A3). Thus, $\sqrt{K}\left\|h_{\bar{S}_{t}^{K}}^{K}-h_{\bar{S}_{t}}^{\infty}\right\|_{\infty} \leqslant c(1+$ $\left.\left\|Z_{t}^{K}\right\|_{W^{-4}}\right)$ for $t \in \mathbb{T}$. Similarly, $\sqrt{K}|| n_{\bar{S}_{t}^{K}}^{i, K}-n_{\bar{S}_{t}}^{i, \infty} \|_{\infty} \leqslant c\left(1+\left\|Z_{t}^{K}\right\|_{W^{-4}}\right)$ for any $i \in \mathbb{K}$. From these two inequalities, we have (25) immediately.

Notice that the operator $L_{S}^{K}$ maps a function from $W^{j}$ to $W^{j-1}$ due to the derivative $f^{\prime}$. We shall write $\mathcal{L}^{j, j^{\prime}}=L\left(W^{j}, W^{j^{\prime}}\right)$ for the space of linear operators from $W^{j}$ to $W^{j^{\prime}}$. Then, we have the following results.

Proposition 10. Suppose (A2) holds. Then we have

$$
\begin{gathered}
\sup _{K, S}\left\|\widehat{L}_{S}^{K}\right\|_{\mathcal{L}^{j, j}} \leqslant c, \quad j \leqslant 3 ; \\
\sup _{K, S}\left\|L_{S}^{K}\right\|_{\mathcal{L}^{j, j-1}} \leqslant c, \quad 2 \leqslant j \leqslant 4 .
\end{gathered}
$$

Proof. First, note that if $f \in C^{j}$ and $g \in W^{j}$, then

$$
\|f g\|_{W^{j}} \leqslant c|| f\left\|_{C^{j}}\right\| g \|_{W^{j}} .
$$

The triangle inequality then yields

$$
\left\|\widehat{L}_{\bar{S}_{t}^{K}}^{K} f\right\|_{W^{j}} \leqslant\left\|h_{\bar{S}_{t}^{K}}^{K}\right\|_{C^{j}}\|f\|_{W^{j}}+\|f\|_{\infty} \sum_{i \in \mathbb{K}}\left\|n_{\bar{S}_{t}^{K}}^{i, K}\right\|_{W^{j}} \leqslant c_{1}\|f\|_{W^{j}}
$$

by (A2) and embedding. Thus, $\left\|\widehat{L}_{\bar{S}_{t}^{K}}^{K}\right\|_{\mathcal{L}^{j, j}} \leqslant c_{1}$ and (ii) follows. For (iii),

$$
\left\|L_{S}^{K} f\right\|_{W^{j-1}}=\left\|f^{\prime}\right\|_{W^{j-1}}+\left\|\widehat{L}_{S}^{K} f\right\|_{W^{j-1}} \leqslant\|f\|_{W^{j}}+c_{1}\|f\|_{W^{j-1}} \leqslant c_{2}\|f\|_{W^{j}},
$$

by (i) and again embedding.

Now, define the operator $\Lambda_{t}^{K}$ as

$$
\Lambda_{t}^{K} f=\sqrt{K}\left(\left(\widehat{L}_{\bar{S}_{t}^{K}}^{K}-\widehat{L}_{\bar{S}_{t}}^{\infty}\right) f, \bar{S}_{t}\right)+\left(L_{\bar{S}_{t}^{K}}^{K} f, Z_{t}^{K}\right) .
$$

Then, Propositions 9 and 10 imply the following.

Corollary 1. Suppose that (A2) and (A3) hold. Let $2 \leqslant j \leqslant 4$. For $t \in \mathbb{T}$,

$$
\left\|\Lambda_{t}^{K}\right\|_{W^{-j}} \leqslant c_{1}\left(1+\left(1, \bar{S}_{0}\right) e^{c_{2} t}\right)\left(1+\left\|Z_{t}^{K}\right\|_{W^{-(j-1)}}\right) .
$$


Proof. Since

$$
\left|\Lambda_{t}^{K} f\right| \leqslant\left(\left|\sqrt{K}\left(\widehat{L}_{\bar{S}_{t}^{K}}^{K}-\widehat{L}_{\bar{S}_{t}}^{\infty}\right) f\right|, \bar{S}_{t}\right)+\left\|L_{\bar{S}_{t}^{K}}^{K} f\right\|_{W^{j-1}}|| Z_{t}^{K} \|_{W^{-(j-1)}},
$$

by Propositions 9 and 10(ii), we have that

$$
\left|\Lambda_{t}^{K} f\right| \leqslant c_{1}\left(1+\left\|Z_{t}^{K}\right\|_{W^{-4}}\right)\|f\|_{W^{j}}\left(1, \bar{S}_{t}\right)+c_{2}\|f\|_{W^{j-1}}\left\|Z_{t}^{K}\right\|_{W^{-(j-1)}} .
$$

Using embedding and the bound

$$
\left(1, \bar{S}_{t}\right) \leqslant\left(1, \bar{S}_{0}\right) e^{c t}
$$

(due to Gronwall's inequality), we have

$$
\left|\Lambda_{t}^{K} f\right| \leqslant c_{3}\left(1+\left\|Z_{t}^{K}\right\|_{W^{-(j-1)}}\right)\|f\|_{W^{j}}\left(1, \bar{S}_{0}\right) e^{c_{4} t}+c_{2}\|f\|_{W^{j}}\left\|Z_{t}^{K}\right\|_{W^{-(j-1)}} .
$$

Rearranging and noting that $x \leqslant 1+x$ for any $x$ complete the proof.

Now, define the operator $\Gamma_{t}^{K}$ as

$$
\begin{aligned}
& \Gamma_{t}^{K} f=\left(\Pi_{\bar{S}_{t}^{K}}^{K} f, \bar{S}_{t}^{K}\right) \\
& =\left(\sum_{i_{1} \in \mathbb{K}} \sum_{i_{2} \in \mathbb{K}} f\left(i_{1}, 0\right) f\left(i_{2}, 0\right) w_{\bar{S}_{t}^{K}}^{i_{1} i_{2}, K}+h_{\bar{S}_{t}^{K}}^{K} f^{2}-2 \sum_{i \in \mathbb{K}} f(i, 0) h_{\bar{S}_{t}^{K}}^{K} \hat{m}_{\bar{S}_{t}^{K}}^{i, K} f, \bar{S}_{t}^{K}\right),
\end{aligned}
$$

and let $\left(p_{l}^{j}\right)_{l \geqslant 1}$ be a complete orthonormal basis of $W^{j}$.

Proposition 11. Let $j \in \mathbb{N}$. Suppose (CO) holds. Then, for $t \in \mathbb{T}$,

$$
\left|\sum_{l \geqslant 1} \Gamma_{t}^{K} p_{l}^{j}\right| \leqslant c\left(1, \bar{S}_{t}^{K}\right)
$$

and for $u \leqslant t$,

$$
\left|\sum_{l \geqslant 1} \Gamma_{u}^{K} \Theta_{t-u} p_{l}^{j}\right| \leqslant c\left(1, \bar{S}_{u}^{K}\right)
$$

Proof. First, note that

$$
\sup _{s_{1} \in \mathbb{S} \sup _{2} \in \mathbb{S}}\left|\sum_{l \geqslant 1} p_{l}^{j}\left(s_{1}\right) p_{l}^{j}\left(s_{2}\right)\right| \leqslant c .
$$

This can be seen by considering the operator $\mathcal{H}_{s}: f \mapsto f(s)$ on $W^{j}$. The Riesz Representation Theorem and Parseval's identity yield $\left\|\mathcal{H}_{s}\right\|_{W^{-j}}^{2}=\sum_{l \geqslant 1}\left(p_{l}^{j}(s)\right)^{2}$ on one hand, and $\left|\mathcal{H}_{s} f\right|=|f(s)| \leqslant\|f\|_{C^{0}} \leqslant c_{1}|| f \|_{W^{j}}$ gives $\left\|\mathcal{H}_{s}\right\|_{W^{-j}} \leqslant c_{1}$ on the other hand. Thus, $\sum_{l \geqslant 1}\left(p_{l}^{j}(s)\right)^{2} \leqslant c_{2}$ for all $s \in \mathbb{S}$. Now, for any $s_{1} \in \mathbb{S}$ and $s_{2} \in \mathbb{S}$,

$$
\left|\sum_{l \geqslant 1} p_{l}^{j}\left(s_{1}\right) p_{l}^{j}\left(s_{2}\right)\right|^{2} \leqslant c_{3}\left\|\sum_{l \geqslant 1} p_{l}^{j}\left(s_{1}\right) p_{l}^{j}\right\|_{W^{j}}^{2}=c_{3} \sum_{l \geqslant 1}\left(p_{l}^{j}\left(s_{1}\right)\right)^{2} \leqslant c_{4} .
$$

Thus, (31) follows. By this and (C0), (29) follows immediately from (28).

For (30), observe that by $(\mathrm{C} 0)$,

$$
\begin{aligned}
\left|\sum_{l \geqslant 1} \Gamma_{u}^{K} \Theta_{t-u} p_{l}^{j}\right| \leqslant c_{5}\left(\sum_{i_{1} \in \mathbb{K}} \sum_{i_{2} \in \mathbb{K}}\left|\sum_{l \geqslant 1} \Theta_{t-u} p_{l}^{j}\left(i_{1}, 0\right) \Theta_{t-u} p_{l}^{j}\left(i_{2}, 0\right)\right|\right. \\
\left.+\left|\sum_{l \geqslant 1}\left(\Theta_{t-u} p_{l}^{j}\right)^{2}\right|+\sum_{i \in \mathbb{K}}\left|\sum_{l \geqslant 1} \Theta_{t-u} p_{l}^{j}(i, 0) \Theta_{t-u} p_{l}^{j}\right|, \bar{S}_{u}^{K}\right),
\end{aligned}
$$


and

$$
\begin{aligned}
& \sup _{s_{1} \in \mathbb{K} \times\left[0, u+a^{*}\right]} \sup _{s_{2} \in \mathbb{K} \times\left[0, u+a^{*}\right]}\left|\sum_{l \geqslant 1} \Theta_{t-u} p_{l}^{j}\left(s_{1}\right) \Theta_{t-u} p_{l}^{j}\left(s_{2}\right)\right| \\
= & \sup _{i_{1} \in \mathbb{K}, w_{1} \in\left[0, u+a^{*}\right]} \sup _{i_{2} \in \mathbb{K}, w_{2} \in\left[0, u+a^{*}\right]}\left|\sum p_{l}^{j}\left(i_{1}, w_{1}+t-u\right) p_{l}^{j}\left(i_{2}, w_{2}+t-u\right)\right| \\
\leqslant & \sup _{s_{1} \in \mathbb{K} \times\left[t-u, t+a^{*}\right]} \sup _{s_{2} \in \mathbb{K} \times\left[t-u, t+a^{*}\right]}\left|\sum_{l \geqslant 1} p_{l}^{j}\left(s_{1}\right) p_{l}^{j}\left(s_{2}\right)\right|,
\end{aligned}
$$

which is finite by (31). Hence, (30) follows.

\section{A.3.3. Bounds on the norm of $Z^{K}$.}

\section{Proposition 12.}

$$
\sup _{t \leqslant T} \sup _{K \geqslant 1} \mathbb{E}\left[\left\|Z_{t}^{K}\right\|_{W^{-2}}\right]<\infty
$$

Proof. For this, we use the alternative representation of $Z^{K}$ as given in Proposition 8. Note that, for $\phi \in W^{2}$,

$$
\begin{aligned}
\left(\phi, Z_{t}^{K}\right)=\left(\Theta_{t} \phi, Z_{0}^{K}\right)+\sqrt{K} \int_{0}^{t}( & \left.\left(\widehat{L}_{\bar{S}_{u}^{K}}^{K}-\widehat{L}_{\bar{S}_{u}}^{\infty}\right) \Theta_{t-u} \phi, \bar{S}_{u}\right) d u \\
& +\int_{0}^{t}\left(\widehat{L}_{\bar{S}_{u}^{K}}^{K} \Theta_{t-u} \phi, Z_{u}^{K}\right) d u+\int_{0}^{t}\left(\Theta_{t-u} \phi, d \tilde{M}_{u}^{K}\right) .
\end{aligned}
$$

Thus, by Propositions 9 and 10, with embedding,

$$
\begin{aligned}
\left|\left(\phi, Z_{t}^{K}\right)\right| \leqslant & \left\|\Theta_{t} \phi\right\|_{W^{2}}\left\|Z_{0}^{K}\right\|_{W^{-2}}+c_{1} \int_{0}^{t}\left(1+\left\|Z_{u}^{K}\right\|_{W^{-2}}\right)\left\|\Theta_{t-u} \phi\right\|_{W^{2}}\left(1, \bar{S}_{u}\right) d u \\
& +c_{2} \int_{0}^{t}\left\|\Theta_{t-u} \phi\right\|_{W^{2}}\left\|Z_{u}^{K}\right\|_{W^{-2}} d u+\left|\int_{0}^{t}\left(\Theta_{t-u} \phi, d \tilde{M}_{u}^{K}\right)\right| \\
\leqslant & c_{3}\|\phi\|_{W^{2}}\left\{\left\|Z_{0}^{K}\right\|_{W^{-2}}+\left(1, \bar{S}_{0}\right) e^{c_{4} t} t\right. \\
& \left.+\left(1+\left(1, \bar{S}_{0}\right) e^{c_{4} t}\right) \int_{0}^{t}\left\|Z_{u}^{K}\right\|_{W^{-2}} d u+\left\|\int_{0}^{t} \Theta_{t-u}^{*} d \tilde{M}_{u}^{K}\right\|_{W^{-2}}\right\}
\end{aligned}
$$

where the last inequality above is due to (27), (22) and that we write $\int_{0}^{t} \Theta_{t-u}^{*} d \tilde{M}_{u}^{K}$ for an operator such that $\left(f, \int_{0}^{t} \Theta_{t-u}^{*} d \tilde{M}_{u}^{K}\right)=\int_{0}^{t}\left(\Theta_{t-u} f, d \tilde{M}_{u}^{K}\right)$, resulting in an expression for $\left\|Z_{t}^{K}\right\|_{W^{-2}}$.

Further, $\mathbb{E}\left[\left\|\int_{0}^{t} \Theta_{t-u}^{*} d \tilde{M}_{u}^{K}\right\|_{W^{-2}}\right] \leqslant \mathbb{E}\left[\left\|\int_{0}^{t} \Theta_{t-u}^{*} d \tilde{M}_{u}^{K}\right\|_{W^{-2}}^{2}\right]^{1 / 2}$. The Riesz Representation Theorem and Parseval's identity yield, for $r \leqslant t$,

$$
\begin{aligned}
\mathbb{E}\left[\left\|\int_{0}^{r} \Theta_{t-u}^{*} d \tilde{M}_{u}^{K}\right\|_{W^{-2}}^{2}\right] & =\mathbb{E}\left[\sum_{l \geqslant 1}\left(\int_{0}^{r}\left(\Theta_{t-u} p_{l}^{2}, d \tilde{M}_{u}^{K}\right)\right)^{2}\right] \\
& =\sum_{l \geqslant 1} \mathbb{E}\left[\left\langle\int_{0}\left(\Theta_{t-u} p_{l}^{2}, d \tilde{M}_{u}^{K}\right)\right\rangle_{r}\right]=\sum_{l \geqslant 1} \mathbb{E}\left[\int_{0}^{r} \Gamma_{u}^{K} \Theta_{t-u} p_{l}^{2} d u\right] .
\end{aligned}
$$


It then follows from (30) and (17) that this is bounded by $c_{5}\left(1, \bar{S}_{0}^{K}\right) e^{c_{6} r} r$. Taking $r=t$, we have $\mathbb{E}\left[\left\|\int_{0}^{t} \Theta_{t-u}^{*} d \tilde{M}_{u}^{K}\right\|_{W^{-2}}^{2}\right] \leqslant c_{5}\left(1, \bar{S}_{0}^{K}\right) e^{c_{6} t} t$. Therefore,

$$
\begin{aligned}
\mathbb{E}\left[\left\|Z_{t}^{K}\right\|_{W^{-2}}\right] \leqslant & c_{7}\left\{\left\|Z_{0}^{K}\right\|_{W^{-2}}+\left(1, \bar{S}_{0}\right) e^{c_{4} T} T^{2}\right. \\
& \left.+\left(1+\left(1, \bar{S}_{0}\right) e^{c_{4} T}\right) \int_{0}^{t} \mathbb{E}\left[\left\|Z_{u}^{K}\right\|_{W^{-2}}\right] d u+\left(1, \bar{S}_{0}^{K}\right)^{1 / 2} e^{c_{8} T} T^{1 / 2}\right\}
\end{aligned}
$$

and the proof is complete by Gronwall's inequality, with (C3) and (A4).

\section{Proposition 13.}

$$
\sup _{K \geqslant 1} \mathbb{E}\left[\sup _{t \leqslant T}\left\|Z_{t}^{K}\right\|_{W^{-3}}\right]<\infty .
$$

Proof. This relies on the bound in Proposition 12, From (8), we have for $f \in W^{3}$,

$$
\begin{aligned}
\left|\left(f, Z_{t}^{K}\right)\right| \leqslant\|f\|_{W^{3}}\left\|Z_{0}^{K}\right\|_{W^{-3}}+\sqrt{K} \int_{0}^{t}\left(\left|L_{\bar{S}_{u}^{K}}^{K} f-L_{\bar{S}_{u}}^{\infty} f\right|, \bar{S}_{u}\right) d u \\
\quad+\int_{0}^{t}\left\|L_{\bar{S}_{u}^{K}}^{K}\right\|_{\mathcal{L}^{3,2}}\|f\|_{W^{3}}\left\|Z_{u}^{K}\right\|_{W^{-2}} d u+\left|\tilde{M}_{t}^{f, K}\right| \\
\leqslant\|f\|_{W^{3}}\left\|Z_{0}^{K}\right\|_{W^{-3}}+c_{1}\|f\|_{W^{3}}\left(1, \bar{S}_{0}\right) e^{c_{2} t} \int_{0}^{t}\left(1+\left\|Z_{u}^{K}\right\|_{W^{-2}}\right) d u \\
+c_{3}\|f\|_{W^{3}} \int_{0}^{t}\left\|Z_{u}^{K}\right\|_{W^{-2}} d u+\|f\|_{W^{3}}\left\|\tilde{M}_{t}^{K}\right\|_{W^{-3}}
\end{aligned}
$$

by Propositions 9 and 10, (27) and embedding. This gives

$$
\begin{aligned}
\sup _{t \leqslant T}\left\|Z_{t}^{K}\right\|_{W^{-3}} \leqslant & \left\|Z_{0}^{K}\right\|_{W^{-3}} \\
& +c_{4}\left(1+\left(1, \bar{S}_{0}\right) e^{c_{2} T}\right) \int_{0}^{T}\left(1+\left\|Z_{u}^{K}\right\|_{W^{-2}}\right) d u+\sup _{t \leqslant T}\left\|\tilde{M}_{t}^{K}\right\|_{W^{-3}} .
\end{aligned}
$$

By the Riesz Representation Theorem and Parseval's identity again, now along with Doob's inequality, we have

$$
\begin{aligned}
\mathbb{E}\left[\sup _{t \leqslant T}\left\|\tilde{M}_{t}^{K}\right\|_{W^{-3}}^{2}\right] & =\mathbb{E}\left[\sup _{t \leqslant T} \sum_{l \geqslant 1}\left(\tilde{M}_{t}^{p_{l}^{3}, K}\right)^{2}\right] \leqslant \sum_{l \geqslant 1} \mathbb{E}\left[\sup _{t \leqslant T}\left(\tilde{M}_{t}^{p_{l}^{3}, K}\right)^{2}\right] \\
& \leqslant 4 \sum_{l \geqslant 1} \mathbb{E}\left[\left\langle\tilde{M}^{p_{l}^{3}, K}\right\rangle_{T}\right]=4 \sum_{l \geqslant 1} \mathbb{E}\left[\int_{0}^{T} \Gamma_{u}^{K} p_{l}^{3} d u\right] \\
& \leqslant c_{5}\left(1, \bar{S}_{0}^{K}\right) e^{c_{6} T} T,
\end{aligned}
$$

where the last inequality follows from (29) and (17). Therefore,

$$
\begin{aligned}
\mathbb{E}\left[\sup _{t \leqslant T}\left\|Z_{t}^{K}\right\|_{W^{-3}}\right] \leqslant c_{7}\left\{\left\|Z_{0}^{K}\right\|_{W^{-3}}\right. \\
\left.\quad+\left(1+\left(1, \bar{S}_{0}\right) e^{c_{2} T}\right) \int_{0}^{T}\left(1+\mathbb{E}\left[\left\|Z_{u}^{K}\right\|_{W^{-2}}\right]\right) d u+\left(1, \bar{S}_{0}^{K}\right)^{1 / 2} e^{c_{8} T} T^{1 / 2}\right\}
\end{aligned}
$$

and (C3), (A4), and Proposition 12 complete the proof. 
A.3.4. Tightness of $Z^{K} . Z^{K}$ is tight in $\mathbb{D}\left(\mathbb{T}, W^{-4}\right)$ if the following conditions are satisfied:

(T1) For every $t \in \mathbb{T},\left(Z_{t}^{K}\right)_{K \geqslant 1}$ is tight in $W^{-4}$, that is, for every $\epsilon>0$, there exists a compact set $\mathcal{C}_{\epsilon}$ such that $\mathbb{P}\left(Z_{t}^{K} \in \mathcal{C}_{\epsilon}\right)>1-\epsilon$ for all $K \geqslant 1$, equivalently, $\mathbb{P}\left(Z_{t}^{K} \notin \mathcal{C}_{\epsilon}\right) \leqslant \epsilon$ for all $K \geqslant 1$.

(T2) Suppose $Z_{t}^{K}=\tilde{V}_{t}^{K}+\tilde{M}_{t}^{K}$. For each $\epsilon_{1}, \epsilon_{2}>0$, there exists $\delta>0$ and $K_{0} \geqslant 1$ such that for every sequence of stopping times $\tau^{K} \leqslant T$,

(T2a) $\sup _{K>K_{0}} \sup _{\zeta<\delta} \mathbb{P}\left(\left\|\tilde{V}_{\tau^{K}+\zeta \wedge T}^{K}-\tilde{V}_{\tau^{K}}^{K}\right\|_{W^{-4}}>\epsilon_{1}\right)<\epsilon_{2}$,

(T2b) $\sup _{K>K_{0}} \sup _{\zeta<\delta} \mathbb{P}\left(\left|\left\langle\left\langle\tilde{M}^{K}\right\rangle\right\rangle_{\tau^{K}+\zeta \wedge T}-\left\langle\left\langle\tilde{M}^{K}\right\rangle\right\rangle_{\tau^{K}}\right|>\epsilon_{1}\right)<\epsilon_{2}$,

where $\left\langle\left\langle\tilde{M}^{K}\right\rangle\right\rangle$ is defined such that $\left(\left\|\tilde{M}_{t}^{K}\right\|_{W^{-4}}^{2}-\left\langle\left\langle\tilde{M}^{K}\right\rangle\right\rangle_{t}\right)_{t \geqslant 0}$ is a martingale.

Proposition 14. Both the sequences $Z^{K}$ and $\tilde{M}^{K}$ are tight in $\mathbb{D}\left(\mathbb{T}, W^{-4}\right)$.

Proof. (T1) follows from Proposition 12, Let $B_{W^{-2}}(R)=\left\{\mu \in W^{-2}:\|\mu\|_{W^{-2}} \leqslant\right.$ $R\}$. Since $W^{-2}$ is Hilbert-Schmidt embedded in $W^{-4}, B_{W^{-2}}(R)$ is compact in $W^{-4}$. From Proposition 12, for any $\epsilon>0$, there exists $R$ such that $\mathbb{P}\left(Z_{t}^{K} \notin B_{W^{-2}}(R)\right) \leqslant \epsilon$ for all $K \geqslant 1$, since $\mathbb{P}\left(Z_{t}^{K} \notin B_{W^{-2}}(R)\right)=\mathbb{P}\left(\left\|Z_{t}^{K}\right\|_{W^{-2}}>R\right) \leqslant \frac{1}{R} \mathbb{E}\left[\left\|Z_{t}^{K}\right\|_{W^{-2}}\right]$.

For $(\mathrm{T} 2 \mathrm{a})$ and $(\mathrm{T} 2 \mathrm{~b})$, we check the stronger conditions established in 7 , Theorem 11]: There exists a $K_{0} \geqslant 1$ such that

$\left(\mathrm{T} 2 \mathrm{a}^{\prime}\right) \sup _{K \geqslant K_{0}} \mathbb{E}\left[\sup _{t \leqslant T}\left\|\Lambda_{t}^{K}\right\|_{W^{-j}}\right] \leqslant c_{T}$,

$\left(\mathrm{T} 2 \mathrm{~b}^{\prime}\right) \sup _{K \geqslant K_{0}} \mathbb{E}\left[\sup _{t \leqslant T}\left|\sum_{l \geqslant 1} \Gamma_{t}^{K} p_{l}^{j}\right|\right] \leqslant c_{T}$,

where $\left(p_{l}^{j}\right)_{l \geqslant 1}$ is a complete orthonormal basis of $W^{j}$.

From Corollary 1, we obtain

$$
\mathbb{E}\left[\sup _{t \leqslant T}\left\|\Lambda_{t}^{K}\right\|_{W^{-4}}\right] \leqslant c_{1}\left(1+\left(1, \bar{S}_{0}\right) e^{c_{2} T}\right)\left(1+\mathbb{E}\left[\sup _{t \leqslant T}\left\|Z_{t}^{K}\right\|_{W^{-3}}\right]\right)
$$

and (T2a') holds as a result of Proposition 13, Finally, (T2b') is a result of (29) and (18).

The tightness of $\tilde{M}^{K}$ follows from (32) and (T2b').

We can further show that $Z^{K}$ and $\tilde{M}^{K}$ are C-tight, that is, the two sequences are tight and all limit points of the sequences are continuous.

Proposition 15. Both the sequences $Z^{K}$ and $\tilde{M}^{K}$ are $C$-tight, all limit points are elements of $\mathbb{C}\left(\mathbb{T}, W^{-4}\right)$.

Proof. As in 7], for C-tightness of $Z^{K}$, we show that (see e.g. 14, Proposition VI 3.26 (iii)]), for all $u \in \mathbb{T}$ and $\epsilon>0, \lim _{K \rightarrow \infty} \mathbb{P}\left(\sup _{t \leqslant u}\left\|\Delta Z_{t}^{K}\right\|_{W^{-4}}>\epsilon\right)=0$. Note that $Z^{K}$ jumps when $S^{K}$ jumps, which occurs when there is a birth or a death. Thus, for $f \in W^{4}$,

$$
\begin{aligned}
\left|\left(f, \Delta Z_{t}^{K}\right)\right| & =\frac{1}{\sqrt{K}}\left|\left(f, S_{t}^{K}-S_{t-}^{K}\right)\right| \\
& \leqslant \frac{1}{\sqrt{K}}\left(\sum_{i \in \mathbb{K}} \sup _{s \in \mathbb{S}}\left|\check{\xi}_{\bar{S}_{t}^{K}}^{i, K}(s) f(i, 0)\right|+\sum_{i \in \mathbb{K}} \sup _{s \in \mathbb{S}}\left|\hat{\xi}_{\bar{S}_{t}^{K}}^{i, K}(s) f(i, 0)-f(s)\right|\right) \\
& \leqslant \frac{c}{\sqrt{K}}|| f \|_{W^{4}}(1+\Xi)
\end{aligned}
$$


by (A1), giving $\left\|\Delta Z_{t}^{K}\right\|_{W^{-4}} \leqslant \frac{c}{\sqrt{K}}(1+\Xi)$. Hence,

$$
\mathbb{P}\left(\sup _{t \leqslant u}\left\|\Delta Z_{t}^{K}\right\|_{W^{-4}}>\epsilon\right) \leqslant \frac{1}{\epsilon} \mathbb{E}\left[\sup _{t \leqslant u}\left\|\Delta Z_{t}^{K}\right\|_{W^{-4}}\right] \leqslant \frac{1}{\epsilon} \frac{c}{\sqrt{K}}(1+\mathbb{E}[\Xi]),
$$

which converges to zero as $K$ tends to infinity.

Observing that $Z^{K}$ and $\tilde{M}^{K}$ have the same discontinuities, i.e. $\Delta Z_{t}^{K}=\Delta \tilde{M}_{t}^{K}$, $\tilde{M}^{K}$ also satisfies the conditions of being C-tight.

A.3.5. Convergence of $\tilde{M}^{K}$ and $Z^{K}$. We now give the final steps in establishing the convergence of $Z^{K}$.

Proposition 16. The sequence $\tilde{M}^{K}$ convergeces weakly to $\tilde{M}^{\infty}$ such that for any $f \in W^{4}, \tilde{M}_{t}^{f, \infty} \equiv\left(f, \tilde{M}_{t}^{\infty}\right), t \in \mathbb{T}$, is a continuous Gaussian martingale with predictable quadratic variation

$$
\begin{aligned}
\left\langle\tilde{M}^{f, \infty}\right\rangle_{t}=\int_{0}^{t}\left(\sum_{i_{1} \in \mathbb{K}} \sum_{i_{2} \in \mathbb{K}} f\left(i_{1}, 0\right) f\left(i_{2}, 0\right) w_{\bar{S}_{u}}^{i_{1} i_{2}, \infty}+h_{\bar{S}_{u}}^{\infty} f^{2}\right. & \\
& \left.-2 \sum_{i \in \mathbb{K}} f(i, 0) h_{\bar{S}_{u}}^{\infty} \hat{m}_{\bar{S}_{u}}^{i, \infty} f, \bar{S}_{u}\right) d u
\end{aligned}
$$

Proof. Same as in [7, this can be achieved by showing that $\tilde{M}^{f, K}$ converges to a continuous Gaussian martingale $\tilde{M}^{f, \infty}$ with predictable quadratic variation (33). In view of the tightness of $\tilde{M}^{K}, \tilde{M}^{K}$ converges to $\tilde{M}^{\infty}$.

Proposition 17. The limiting process $z$ of the sequence $Z^{K}$ satisfies (9) for any $f \in W^{4}$ and $t \in \mathbb{T}$.

Proof. Every limit point $z$ of the sequence $Z^{K}$ satisfies, for $\phi \in W^{4}$ and $t \in \mathbb{T}$,

$$
\begin{gathered}
\left(\phi, z_{t}\right)=\left(\Theta_{t} \phi, z_{0}\right)+\int_{0}^{t}\left(-\partial_{S} h_{\bar{S}_{u}}^{\infty}\left(z_{u}\right) \Theta_{t-u} \phi+\sum_{i \in \mathbb{K}} \Theta_{t-u} \phi(i, 0) \partial_{S} n_{\bar{S}_{u}}^{i, \infty}\left(z_{u}\right), \bar{S}_{u}\right) d u \\
+\int_{0}^{t}\left(-h_{\bar{S}_{u}}^{\infty} \Theta_{t-u} \phi+\sum_{i \in \mathbb{K}} \Theta_{t-u} \phi(i, 0) n_{\bar{S}_{u}}^{i, \infty}, z_{u}\right) d u+\int_{0}^{t}\left(\Theta_{t-u} \phi, d \tilde{M}_{u}^{\infty}\right) .
\end{gathered}
$$

This can be established by showing the convergence of each and every term of (23). Furthermore, if $z^{1}$ and $z^{2}$ both are solutions to (34) with $z_{0}^{1}=z_{0}^{2}$, by showing that $\left\|z_{t}^{1}-z_{t}^{2}\right\|_{W^{-4}}=0$ for $t \in \mathbb{T}$, we have the uniqueness. Lastly, we note that (34) is equivalent to (9), which is followed by Fubini's theorem and that $\Theta_{t-u} \phi(s)=\phi(s)+\int_{u}^{t} \Theta_{w-u} \phi^{\prime}(s) d w$.

\section{A.4. Proof of Proposition 1 ,}

Proof. Using representation (34) and noting that $\mathbb{E}\left[\int_{0}^{t}\left(\Theta_{t-u} \phi, d \tilde{M}_{u}^{\infty}\right)\right]=0$, with $\nu_{t}: f \mapsto \mathbb{E}\left[\left(f, Z_{t}\right)\right]$, we have for $\phi \in W^{4}$,

$$
\begin{aligned}
\left(\phi, \nu_{t}\right)=\left(\Theta_{t} \phi, \nu_{0}\right)+\int_{0}^{t}( & \left.-\left(g_{\bar{S}_{u}, *}^{h}, \nu_{u}\right) \Theta_{t-u} \phi+\sum_{i \in \mathbb{K}} \Theta_{t-u} \phi(i, 0)\left(g_{\bar{S}_{u}, *}^{n, i}, \nu_{u}\right), \bar{S}_{u}\right)_{*} d u \\
& +\int_{0}^{t}\left(-h_{\bar{S}_{u}}^{\infty} \Theta_{t-u} \phi+\sum_{i \in \mathbb{K}} \Theta_{t-u} \phi(i, 0) n_{\bar{S}_{u}, \infty}^{i,}, \nu_{u}\right) d u
\end{aligned}
$$


Thus, under the assumptions in the statement and (A2),

$$
\left\|\nu_{t}\right\|_{W^{-4}} \leqslant\left\|\nu_{0}\right\|_{W^{-4}}+c_{1}\left(1+\left(1, \bar{S}_{0}\right) e^{c_{2} t}\right) \int_{0}^{t}\left\|\nu_{u}\right\|_{W^{-4}} d u .
$$

Gronwall's inequality then gives

$$
\left\|\nu_{t}\right\|_{W^{-4}} \leqslant\left\|\nu_{0}\right\|_{W^{-4}} e^{c_{1}\left(1+\left(1, \bar{S}_{0}\right) e^{c_{2} T}\right)^{T}} .
$$

Now, let $\left(\phi_{m}\right)_{m}$ be a sequence of functions in $C^{\infty}$ converging to $\phi \in C^{0}$. By dominated convergence theorem, each term in (35) with $\phi_{m}$ converges. Thus, (35) holds for $\phi \in C^{0}$. Moreover, $\nu_{t}$ is a bounded linear operator. Therefore, $\nu_{t} \in C^{-0}$; in other words, $\nu_{t}$ defines a signed measure.

\section{Appendix B. Appendices for Section 3}

\section{B.1. Semimartingale representation of the serial monogamy mating sys-}

tem. To obtain an equation for the population structure in the serial monogamy mating system, we need to define a few more terms. Let $R(v, w, t)$ be the number of marriages by time $t$ with females at ages not greater than $v$ and males at ages not greater than $w$. Let $Q^{1}(v, w, t)$ (resp. $Q^{2}(v, w, t)$ ) be the number of cases by time $t$ where female (resp. male) partners died at age not greater than $v$ when their male (resp. female) partners were at age not greater than $w$, and let $Q^{3}(v, w, t)$ count the number of events by time $t$ where couples are separated while both of the mates are alive and with ages not greater than $v$ and $w$. We also let $D^{\circ}(v, t)$ (resp. $D^{\sigma^{*}}(v, t)$ ) be the number of single females (resp. males) who died by time $t$ at age not greater that $v$, and $B^{\circ}(t)$ (resp. $\left.B^{\sigma}(t)\right)$ be the number of females (reps. males) born by time $t$. Then we have, for test functions $f$ as specified on Section 3.1.

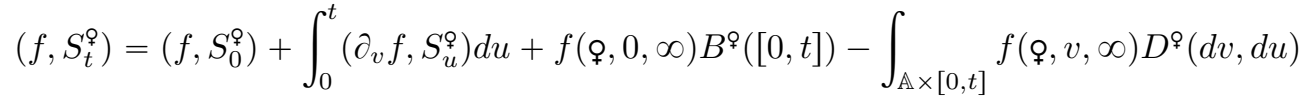

$$
\begin{aligned}
& +\int_{\mathbb{A} \times \mathbb{A} \times[0, t]} f(q, v, \infty) Q^{2}(d v, d w, d u)+\int_{\mathbb{A} \times \mathbb{A} \times[0, t]} f(q, v, \infty) Q^{3}(d v, d w, d u) \\
& -\int_{\mathbb{A} \times \mathbb{A} \times[0, t]} f(q, v, \infty) R(d v, d w, d u), \\
& \left(f, S_{t}^{\sigma^{\circ}}\right)=\left(f, S_{0}^{\sigma^{*}}\right)+\int_{0}^{t}\left(\partial_{w} f, S_{u}^{\sigma^{\prime}}\right) d u+f\left(\sigma^{\star \prime}, \infty, 0\right) B^{\sigma^{\prime}}([0, t])-\int_{\mathbb{A} \times[0, t]} f\left(\sigma^{\star \prime}, \infty, w\right) D^{\sigma^{\circ}}(d v, d u) \\
& +\int_{\mathbb{A} \times \mathbb{A} \times[0, t]} f\left(\sigma^{\prime \prime}, \infty, w\right) Q^{1}(d v, d w, d u)+\int_{\mathbb{A} \times \mathbb{A} \times[0, t]} f\left(\sigma^{\prime \prime}, \infty, w\right) Q^{3}(d v, d w, d u) \\
& -\int_{\mathbb{A} \times \mathbb{A} \times[0, t]} f\left(\sigma^{\prime \prime}, \infty, w\right) R(d v, d w, d u), \\
& \left(f, S_{t}^{\Phi^{\circ}}\right)=\left(f, S_{0}^{\Phi^{\circ}}\right)+\int_{0}^{t}\left(\partial_{v} f+\partial_{w} f, S_{u}^{\Phi^{\circ}}\right) d u-\int_{\mathbb{A} \times \mathbb{A} \times[0, t]} f\left(\Phi^{\prime}, v, w\right) Q^{3}(d v, d w, d u) \\
& -\int_{\mathbb{A} \times \mathbb{A} \times[0, t]} f\left(\Phi^{\prime}, v, w\right) Q^{1}(d v, d w, d u)-\int_{\mathbb{A} \times \mathbb{A} \times[0, t]} f\left(\Phi^{*}, v, w\right) Q^{2}(d v, d w, d u) \\
& +\int_{\mathbb{A} \times \mathbb{A} \times[0, t]} f\left(\Phi^{\prime}, v, w\right) R(d v, d w, d u) .
\end{aligned}
$$


Compensating the birth, death and marriage terms, we obtain a semimartingale representation for each of the three types, which can be combined and written as (10). For instance, the following processes are local martingales:

$$
\begin{aligned}
& B^{i}([0, t])-\int_{0}^{t}\left(b m^{i}, S_{u}^{\Phi}\right) d u-\int_{0}^{t}\left(b m^{i}, S_{u}^{\odot}\right) d u, \\
& \int_{\mathbb{A} \times[0, t]} g(v) D^{i}(d v, d u)-\int_{0}^{t}\left(h^{i} g, S_{u}^{i}\right) d u, \\
& \int_{\mathbb{A} \times \mathbb{A} \times[0, t]} g(v, w) R(d v, d w, d u)-\int_{0}^{t}\left(\left(\rho(\cdot, *) g(\cdot, *), S_{u}^{\odot}\right) ., S_{u}^{\sigma}\right)_{*} d u, \\
& \int_{\mathbb{A} \times \mathbb{A} \times[0, t]} g(v, w) Q^{i}(d v, d w, d u)-\int_{0}^{t}\left(h^{i} g, S_{u}^{\Phi}\right) d u .
\end{aligned}
$$

The predictable quadratic variation of the martingale can also be obtained in the usual way as in Section A.1.2.

B.2. Total population size in serial monogamy mating system. Before we proceed to establish the tightness of $\left\{\bar{S}_{t}^{K}\right\}$, we first consider the total population size, by taking the test function $f(i, v, w)=\mathbf{1}_{\varphi}(i)+\mathbf{1}_{\sigma^{\circ}}(i)+2 \mathbf{1}_{\varphi^{\circ}}(i)$. This gives a simple dynamics equation that can be easily analysed and controlled, the results of which will be useful in establishing the tightness of $\left\{\bar{S}_{t}^{K}\right\}$.

With a slight abuse of notation, write $X_{t}=\left(\mathbf{1}_{\wp}+\mathbf{1}_{\sigma^{\prime}}+2 \mathbf{1}_{\varphi^{\prime}}, S_{t}\right)$ and $\bar{X}_{t}^{K}=X_{t}^{K} / K$. Note that we have

$$
\begin{aligned}
& \bar{X}_{t}^{K}=\bar{X}_{0}^{K}-\int_{0}^{t}\left(h_{\bar{S}_{u}^{K}}^{\wp, K} \mathbf{1}_{\wp}+h_{\bar{S}_{u}^{K}}^{\sigma, K} \mathbf{1}_{\sigma^{\ddagger}}-\left(h_{\bar{S}_{u}^{K}}^{\wp, K}+h_{\bar{S}_{u}^{K}}^{\sigma^{K}, K}\right) \mathbf{1}_{\phi \wp}, \bar{S}_{u}^{K}\right) d u \\
& +\int_{0}^{t}\left(\left(m_{\bar{S}_{u}^{K}}^{\varphi^{K}}+m_{\bar{S}_{u}^{K}}^{\sigma, K}\right) b_{\bar{S}_{u}^{K}}^{K}\left(\mathbf{1}_{\varphi^{\prime}}+\mathbf{1}_{q}\right), \bar{S}_{u}^{K}\right) d u+\bar{M}_{t}^{X, K},
\end{aligned}
$$

with

$$
\begin{aligned}
& \left\langle\bar{M}^{X, K}\right\rangle_{t}=\int_{0}^{t} \frac{1}{K}\left(h_{\bar{S}_{u}^{K}}^{\rho, K} \mathbf{1}_{\uparrow}+h_{\bar{S}_{u}^{K}}^{\sigma, K} \mathbf{1}_{\sigma^{\sigma}}+\left(h_{\bar{S}_{u}^{K}}^{\rho, K}+h_{\bar{S}_{u}^{K}}^{\sigma, K}\right) \mathbf{1}_{\phi^{\circ}}, \bar{S}_{u}^{K}\right) d u
\end{aligned}
$$

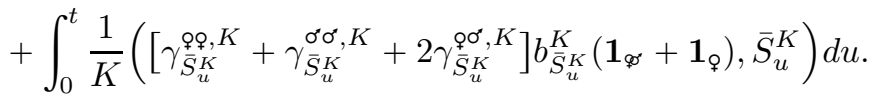

Since the reproduction parameters are bounded, by Gronwall's inequality, we have $\mathbb{E}\left[\bar{X}_{t}^{K}\right] \leqslant \bar{X}_{0}^{K} e^{c t}$ and thus $\sup _{K} \mathbb{E}\left[\bar{X}_{t}^{K}\right]<\infty$. In fact, we can further show that

$$
\sup _{K} \mathbb{E}\left[\sup _{t \in \mathbb{T}} \bar{X}_{t}^{K}\right]<\infty .
$$

From (36), notice that $\left(1, \bar{S}_{u}^{K}\right) \leqslant \bar{X}_{u}^{K}$, we have

$$
\mathbb{E}\left[\sup _{t \in \mathbb{T}} \bar{X}_{t}^{K}\right] \leqslant \bar{X}_{0}^{K}+c_{1} \int_{0}^{T} \mathbb{E}\left[\bar{X}_{u}^{K}\right] d u+\mathbb{E}\left[\sup _{t \in \mathbb{T}} \bar{M}_{t}^{X, K}\right] .
$$

Apply Jensen's and Doob's inequalities,

$$
\begin{aligned}
\mathbb{E}\left[\sup _{t \in \mathbb{T}} \bar{M}_{t}^{X, K}\right]^{2} \leqslant & \mathbb{E}\left[\sup _{t \in \mathbb{T}}\left(\bar{M}_{t}^{X, K}\right)^{2}\right] \leqslant 4 \mathbb{E}\left[\left\langle\bar{M}^{X, K}\right\rangle_{T}\right] \\
& \leqslant c_{2} \frac{1}{K} \int_{0}^{T} \mathbb{E}\left[\bar{X}_{u}^{K}\right] d u \leqslant c_{2} \frac{1}{K} \int_{0}^{T} \bar{X}_{0}^{K} e^{c u} d u \leqslant c_{2} \frac{1}{K} \bar{X}_{0}^{K} e^{c T} T
\end{aligned}
$$


and thus

$$
\mathbb{E}\left[\sup _{t \in \mathbb{T}} \bar{X}_{t}^{K}\right] \leqslant \bar{X}_{0}^{K}+c_{1} \int_{0}^{T} \bar{X}_{0}^{K} e^{c u} d u+\left(c_{2} \frac{1}{K} \bar{X}_{0}^{K} e^{c T} T\right)^{1 / 2},
$$

which gives (37).

In a similar way, we can bound $\mathbb{E}\left[\left(\bar{X}_{t}^{K}\right)^{2}\right]$ and show that

$$
\sup _{K} \mathbb{E}\left[\sup _{t \in \mathbb{T}}\left(\bar{X}_{t}^{K}\right)^{2}\right]<\infty .
$$

\section{B.3. Proof of the Law of Large Numbers (serial monogamy mating sys-} tem).

Proposition 18. The sequence $\left\{\bar{S}_{t}^{K}\right\}$ is tight in $\mathbb{D}(\mathbb{T}, \mathcal{M})$.

Proof. The tightness is obtained by establishing (J1) and (J2). Observe that

$$
\mathbb{P}\left(\sup _{t \in \mathbb{T}}\left(1, \bar{S}_{t}^{K}\right)>\delta\right) \leqslant \frac{1}{\delta} \mathbb{E}\left[\sup _{t \in \mathbb{T}}\left(1, \bar{S}_{t}^{K}\right)\right] \leqslant \frac{1}{\delta} \mathbb{E}\left[\sup _{t \in \mathbb{T}} X_{t}^{K}\right] \leqslant \frac{c}{\delta} .
$$

Thus, for each $\eta$, there exists $\delta_{\eta}$ such that $\mathbb{P}\left(\sup _{t \in \mathbb{T}}\left(1, S_{t}^{K}\right)>\delta_{\eta}\right) \leqslant \eta$. Therefore (J1) holds with compact set

$$
C\left(\delta_{\eta}\right)=\left\{\mu \in \sigma^{\prime \prime}:(1, \mu) \leqslant \delta_{\eta}\right\} .
$$

For (J2a), we have from the semimartingale representation of $\left(f, \bar{S}_{t}^{K}\right)$,

$$
\mathbb{E}\left[\left(f, \bar{S}_{t}^{K}\right)\right] \leqslant c_{1}\|f\|_{C^{1,1}}\left\{\left(1, \bar{S}_{0}^{K}\right)+\int_{0}^{t}\left(\mathbb{E}\left[\left(1, \bar{S}_{u}^{K}\right)\right]+\mathbb{E}\left[\left(1, \bar{S}_{u}^{K}\right)^{2}\right]\right) d u+\mathbb{E}\left[\left|\bar{M}_{t}^{f, K}\right|\right]\right\}
$$

and

$$
\begin{aligned}
\mathbb{E}\left[\left|\bar{M}_{t}^{f, K}\right|\right]^{2} \leqslant \mathbb{E}\left[\left|\bar{M}_{t}^{f, K}\right|^{2}\right]= & \mathbb{E}\left[\left\langle\bar{M}^{f, K}\right\rangle_{t}\right] \\
& \leqslant c_{2}\|f\|_{C^{1,1}}^{2} \frac{1}{K} \int_{0}^{t}\left(\mathbb{E}\left[\left(1, \bar{S}_{u}^{K}\right)\right]+\mathbb{E}\left[\left(1, \bar{S}_{u}^{K}\right)^{2}\right]\right) d u .
\end{aligned}
$$

Notice that

$$
\mathbb{E}\left[\left(1, \bar{S}_{u}^{K}\right)\right]+\mathbb{E}\left[\left(1, \bar{S}_{u}^{K}\right)^{2}\right] \leqslant \mathbb{E}\left[\bar{X}_{u}^{K}\right]+\mathbb{E}\left[\left(\bar{X}_{u}^{K}\right)^{2}\right] \leqslant c_{T}
$$

from the previous section. Therefore, (J2a) follows by Markov's inequality.

Now, let $\zeta \leqslant \delta$ and $\tau^{K}$ be a sequence of stopping times bounded by $T$. We have

$$
\mathbb{E}\left[\left|\bar{V}_{\tau^{K}+\zeta \wedge T}^{f, K}-\bar{V}_{\tau^{K}}^{f, K}\right|\right] \leqslant c_{3}\|f\|_{C^{1,1}} \mathbb{E}\left[\int_{\tau^{K}}^{\tau^{K}+\zeta \wedge T}\left(\left(1, \bar{S}_{u}^{K}\right)+\left(1, \bar{S}_{u}^{K}\right)^{2}\right) d u\right]
$$

and

$$
\begin{gathered}
\mathbb{E}\left[\int_{\tau^{K}}^{\tau^{K}+\zeta \wedge T}\left(\left(1, \bar{S}_{u}^{K}\right)+\left(1, \bar{S}_{u}^{K}\right)^{2}\right) d u\right] \leqslant \int_{0}^{\zeta} \mathbb{E}\left[\left(1, \bar{S}_{\tau^{K}+u \wedge T}^{K}\right)+\left(1, \bar{S}_{\tau^{K}+u \wedge T}^{K}\right)^{2}\right] d u \\
\leqslant \int_{0}^{\zeta}\left(\mathbb{E}\left[\sup _{r \leqslant T}\left(1, \bar{S}_{r}^{K}\right)\right]+\mathbb{E}\left[\sup _{r \leqslant T}\left(1, \bar{S}_{r}^{K}\right)^{2}\right]\right) d u \\
\leqslant \int_{0}^{\zeta}\left(\mathbb{E}\left[\sup _{r \leqslant T} \bar{X}_{r}^{K}\right]+\mathbb{E}\left[\sup _{r \leqslant T}\left(\bar{X}_{r}^{K}\right)^{2}\right]\right) d u \leqslant c_{T} \delta
\end{gathered}
$$


where the last inequality follows from the results in the previous section. Similarly,

$$
\begin{aligned}
& \mathbb{E}\left[\left|\left\langle\bar{M}^{f, K}\right\rangle_{\tau^{K}+\zeta \wedge T}-\left\langle\bar{M}^{f, K}\right\rangle_{\tau^{K}}\right|\right] \\
& \quad \leqslant c_{4}\|f\|_{C^{1,1}}^{2} \frac{1}{K} \mathbb{E}\left[\int_{\tau^{K}}^{\tau^{K}+\zeta \wedge T}\left(\left(1, \bar{S}_{u}^{K}\right)+\left(1, \bar{S}_{u}^{K}\right)^{2}\right) d u\right] \leqslant c_{T^{\prime}}^{\prime} \delta\|f\|_{C^{1,1}}^{2} .
\end{aligned}
$$

(J2b) thus follows by Markov's inequality.

We see that the martingale $\bar{M}^{f, K}$ converges to 0 , since its predictable quadratic variation vanishes.

of Theorem $3-L L N$. Suppose that $\mathcal{S}$ is a limit point of $\bar{S}^{K}$. Note that for any bounded function $f$ and reproduction parameter $q=h^{\circ}, h^{\sigma^{*}}, h^{థ \sigma}, b m^{\circ}, b m^{\sigma^{*}}$,

$$
\begin{aligned}
\mid\left(f q_{\bar{S}_{u}^{K}}^{K}, \bar{S}_{u}^{K}\right)-( & \left.f q_{\mathcal{S}_{u}}^{\infty}, \mathcal{S}_{u}\right)|\leqslant|\left(f\left(q_{\bar{S}_{u}^{K}}^{K}-q_{\mathcal{S}_{u}}^{\infty}\right), \bar{S}_{u}^{K}\right)|+|\left(f q_{\mathcal{S}_{u}}^{\infty}, \bar{S}_{u}^{K}-\mathcal{S}_{u}\right) \mid \\
& \leqslant\|f\|_{\infty}\left\|q_{\bar{S}_{u}^{K}}^{K}-q_{\mathcal{S}_{u}}^{\infty}\right\|_{\infty}\left(1, \bar{S}_{u}^{K}\right)+\|f\|_{\infty}\left\|q_{\mathcal{S}_{u}}^{\infty}\right\|_{\infty}\left\|\bar{S}_{u}^{K}-\mathcal{S}_{u}\right\|
\end{aligned}
$$

converges to zero as $K \rightarrow \infty$. Indeed, the first term on the right hand side vanishes since

$$
\left\|q_{\bar{S}_{u}^{K}}^{K}-q_{\mathcal{S}_{u}}^{\infty}\right\|_{\infty} \leqslant\left\|q_{\bar{S}_{u}^{K}}^{K}-q_{\mathcal{S}_{u}}^{K}\right\|_{\infty}+\left\|q_{\mathcal{S}_{u}}^{K}-q_{\mathcal{S}_{u}}^{\infty}\right\|_{\infty} \rightarrow 0
$$

due to (C1') and (C2'); clearly, the last term in (38) also vanishes. Similarly,

$$
\begin{aligned}
& \left|\left(\left(g(\bullet, *) K \rho_{\bar{S}_{u}^{K}}^{K}(\bullet, *) \mathbf{1}_{\varphi}, \bar{S}_{u}^{K}\right) . \mathbf{1}_{\sigma^{*}}, \bar{S}_{u}^{K}\right)_{*}-\left(\left(g(\bullet, *) \rho_{\mathcal{S}_{u}}^{\infty}(\bullet, *) \mathbf{1}_{\varphi}, \mathcal{S}_{u}\right) . \mathbf{1}_{\sigma^{*}}, \mathcal{S}_{u}\right)_{*}\right| \\
& \leqslant\left|\left(\left(g(\bullet, *) K \rho_{\bar{S}_{u}^{K}}^{K}(\bullet, *) \mathbf{1}_{\varphi}, \bar{S}_{u}^{K}\right) . \mathbf{1}_{\sigma^{*}}, \bar{S}_{u}^{K}\right)_{*}-\left(\left(g(\bullet, *) \rho_{\mathcal{S}_{u}}^{\infty}(\bullet, *) \mathbf{1}_{\odot}, \bar{S}_{u}^{K}\right) . \mathbf{1}_{\sigma^{*}}, \bar{S}_{u}^{K}\right)_{*}\right| \\
& +\left|\left(\left(g(\cdot, *) \rho_{\mathcal{S}_{u}}^{\infty}(\bullet, *) \mathbf{1}_{\wp}, \bar{S}_{u}^{K}\right) . \mathbf{1}_{\sigma^{*}}, \bar{S}_{u}^{K}\right)_{*}-\left(\left(g(\bullet, *) \rho_{\mathcal{S}_{u}}^{\infty}(\bullet, *) \mathbf{1}_{\wp}, \mathcal{S}_{u}\right) . \mathbf{1}_{\sigma^{*}}, \bar{S}_{u}^{K}\right)_{*}\right| \\
& +\left|\left(\left(g(\bullet, *) \rho_{\mathcal{S}_{u}}^{\infty}(\bullet, *) \mathbf{1}_{\wp}, \mathcal{S}_{u}\right) . \mathbf{1}_{\sigma^{*}}, \bar{S}_{u}^{K}\right)_{*}-\left(\left(g(\bullet, *) \rho_{\mathcal{S}_{u}}^{\infty}(\bullet, *) \mathbf{1}_{\wp}, \mathcal{S}_{u}\right) . \mathbf{1}_{\sigma^{*}}, \mathcal{S}_{u}\right)_{*}\right| \\
& \leqslant\|g\|_{\infty}\left\|K \rho_{\bar{S}_{u}^{K}}^{K}-\rho_{\mathcal{S}_{u}}^{\infty}\right\|_{\infty}\left(1, \bar{S}_{u}^{K}\right)^{2}+\|g\|_{\infty}\left\|\rho_{\mathcal{S}_{u}}^{\infty}\right\|_{\infty}\left\|\bar{S}_{u}^{K}-\mathcal{S}_{u}\right\|\left(1, \bar{S}_{u}^{K}\right) \\
& +\|g\|_{\infty}\left\|\rho_{\mathcal{S}_{u}}^{\infty}\right\|_{\infty}\left(1, \mathcal{S}_{u}\right)\left\|\bar{S}_{u}^{K}-\mathcal{S}_{u}\right\|,
\end{aligned}
$$

can be shown vanishing as $K \rightarrow \infty$ using the trick as above with (C1') and (C2'), and that $\mathcal{S}_{u}$ is a limit point of $\bar{S}_{u}^{K}$. Thus limit point of $\bar{S}^{K}$ satisfies (11).

It remains to show that the limit is unique. This is done by considering (11) with test functions that depend also on time, $f(i, v, w, t)$. Now, fix $t \in \mathbb{T}$, for $u \leqslant t$, take $f$ of the form $f(i, v, w, u)=\phi(i, v+t-u, w+t-u)=\widehat{\Theta}_{t-u} \phi(i, v, w)$ as in Proposition [6] Then, $\mathcal{S}$ is shown to satisfy the following equation:

$$
\begin{aligned}
& \left(\phi, \mathcal{S}_{t}\right)=\left(\widehat{\Theta}_{t} \phi, \mathcal{S}_{0}\right)-\int_{0}^{t}\left(\widehat{\Theta}_{t-u} \phi h_{\mathcal{S}_{u}, \infty} \mathbf{1}_{\wp}+\widehat{\Theta}_{t-u} \phi h_{\mathcal{S}_{u}}^{\sigma^{\prime}, \infty} \mathbf{1}_{\sigma^{*}}, \mathcal{S}_{u}\right) d u \\
& +\int_{0}^{t}\left(\left[\left(\widehat{\Theta}_{t-u} \phi\left(\sigma^{\prime \prime}, \infty, \cdot\right)-\widehat{\Theta}_{t-u} \phi\left(\phi^{\prime \prime}, \cdot, \cdot\right)\right) h_{\mathcal{S}_{u}, \infty}+\left(\widehat{\Theta}_{t-u} \phi(\propto, \cdot, \infty)-\widehat{\Theta}_{t-u} \phi\left(\Phi^{\prime}, \cdot, \cdot\right)\right) h_{\mathcal{S}_{u}, \infty}^{\sigma^{\prime},}\right] \mathbf{1}_{\phi^{\prime}}, \mathcal{S}_{u}\right) d u \\
& +\int_{0}^{t}\left(\left(\widehat{\Theta}_{t-u} \phi(\propto, \cdot, \infty)+\widehat{\Theta}_{t-u} \phi\left(\sigma^{\prime \prime}, \infty, \cdot\right)-\widehat{\Theta}_{t-u} \phi(\Phi, \cdot, \cdot)\right) h_{\mathcal{S}_{u}, \infty} \mathbf{1}_{\phi}, \mathcal{S}_{u}\right) d u \\
& +\int_{0}^{t}\left(\left[\widehat{\Theta}_{t-u} \phi(\propto, 0, \infty) m_{\mathcal{S}_{u}, \infty}+\widehat{\Theta}_{t-u} \phi\left(\sigma^{\prime \prime}, \infty, 0\right) m_{\mathcal{S}_{u}}^{\sigma^{\prime \prime} \infty}\right] b_{\mathcal{S}_{u}}^{\infty}\left(\mathbf{1}_{\phi}+\mathbf{1}_{\wp}\right), \mathcal{S}_{u}\right) d u \\
& \left.-\int_{0}^{t}\left(\left(\left[\widehat{\Theta}_{t-u} \phi(\propto, \cdot, \infty)+\widehat{\Theta}_{t-u} \phi\left(\sigma^{\prime \prime}, \infty, *\right)-\widehat{\Theta}_{t-u} \phi\left(\phi^{\prime \prime}, \cdot, *\right)\right] \rho_{\mathcal{S}_{u}}^{\infty}(\cdot, *) \mathbf{1}_{q}, \mathcal{S}_{u}\right) \mathbf{1}_{\sigma^{*}}, \mathcal{S}_{u}\right)\right)_{*} d u \text {. }
\end{aligned}
$$


The uniqueness of the limit is then achieved by considering two processes $\mathcal{S}^{1}$ and $\mathcal{S}^{2}$ that are both solutions to (39) with the same initial point $\mathcal{S}_{0}^{1}=\mathcal{S}_{0}^{2}$. Then we show that $\left|\left(\phi, \mathcal{S}_{t}^{1}-\mathcal{S}_{t}^{2}\right)\right|$ is bounded by $c_{T}\|\phi\|_{\infty}\left\|\mathcal{S}_{u}^{1}-\mathcal{S}_{u}^{2}\right\|$. Therefore, $\mathcal{S}^{1}=\mathcal{S}^{2}$ by Gronwall's inequality.

\section{B.4. Proof of the Central Limit Theorem (serial monogamy mating sys-}

tem). The mechanism in proving the Central Limit Theorem in this serial monogamy mating system would be slightly different from that in Section A.3 due to the iterative bracket, coming from the coupling. To overcome the issue arose from the coupling, we need the stopping times

$$
\tau_{N}^{K}=\inf \left\{t \in \mathbb{T}: \bar{X}_{t}^{K}>N\right\}
$$

As before, we obtain an alternative representation equation for $Z^{K}$. This is done by extending (12) to have test function depending on time, $f=f(i, v, w, t)$ and then, with fixed $t$, taking $f(i, v, w, u)=\phi(i, v+t-u, w+t-u)=\Theta_{t-u} \phi(i, v, w)$ for $u \leqslant t$ and $\phi \in W^{-4}$ as in Remark [5. We then have

$$
\begin{aligned}
& \left(\phi, Z_{t}^{K}\right)=\left(\Theta_{t} \phi, Z_{0}^{K}\right)
\end{aligned}
$$

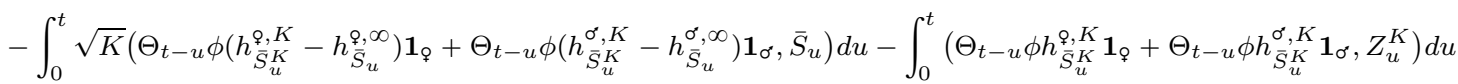

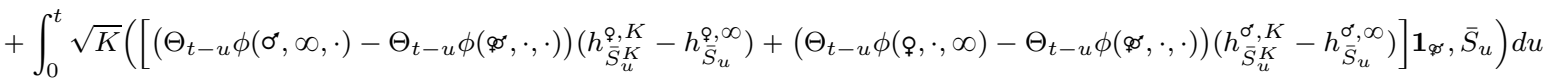

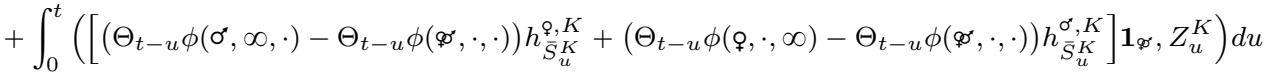

$$
\begin{aligned}
& +\int_{0}^{t} \sqrt{K}\left(\left(\Theta_{t-u} \phi(q, \cdot, \infty)+\Theta_{t-u} \phi\left(\sigma^{\circ}, \infty, \cdot\right)-\Theta_{t-u} \phi(\Phi, \cdot, \cdot)\right)\left(h_{\bar{S}_{u}^{K}}^{\infty, K}-h_{\bar{S}_{u}, \infty}^{\infty,}\right) \mathbf{1}_{\infty}, \bar{S}_{u}\right) d u \\
& +\int_{0}^{t}\left(\left(\Theta_{t-u} \phi(\phi, \cdot, \infty)+\Theta_{t-u} \phi\left(\sigma^{*}, \infty, \cdot\right)-\Theta_{t-u} \phi\left(\Phi^{\prime}, \cdot, \cdot\right)\right) h_{\bar{S}_{u}^{K}}^{\Phi, K} \mathbf{1}_{\Phi}, Z_{u}^{K}\right) d u \\
& +\int_{0}^{t} \sqrt{K}\left(\left[\Theta_{t-u} \phi(q, 0, \infty)\left(b_{\bar{S}_{u}^{K}}^{K} m_{\bar{S}_{u}^{K}}^{\varphi, K}-b_{\bar{S}_{u}}^{\infty} m_{\bar{S}_{u}}^{\rho, \infty}\right)+\Theta_{t-u} \phi\left(\sigma^{\alpha}, \infty, 0\right)\left(b_{\bar{S}_{u}^{K}}^{K} m_{\bar{S}_{u}^{K}}^{\sigma^{\prime} K}-b_{\bar{S}_{u}}^{\infty} m_{\bar{S}_{u}}^{\sigma, \infty}\right)\right]\left(\mathbf{1}_{\phi}+\mathbf{1}_{\varphi}\right), \bar{S}_{u}\right) d u \\
& +\int_{0}^{t}\left(\left[\Theta_{t-u} \phi(\wp, 0, \infty) m_{\bar{S}_{u}^{K K}}^{\rho, K}+\Theta_{t-u} \phi\left(\sigma^{\circ}, \infty, 0\right) m_{\tilde{S}_{u}^{K}}^{\sigma, K}\right] b_{\bar{S}_{u}^{K}}^{K}\left(\mathbf{1}_{\phi^{\sigma}}+\mathbf{1}_{\varphi}\right), Z_{u}^{K}\right) d u \\
& -\int_{0}^{t} \sqrt{K}\left(\left(\left[\Theta_{t-u} \phi(\phi, \cdot, \infty)+\Theta_{t-u} \phi\left(\sigma^{\prime}, \infty, *\right)-\Theta_{t-u} \phi\left(\phi^{\prime}, \cdot, *\right)\right]\left(K \rho_{S_{u}^{K}}^{K}(\cdot, *)-\rho_{\bar{S}_{u}}^{\infty}(\cdot, *)\right) \mathbf{1}_{\varphi}, \bar{S}_{u}\right) . \mathbf{1}_{\sigma^{\circ}}, \bar{S}_{u}\right)_{*} d u \\
& -\int_{0}^{t}\left(\left(\left[\Theta_{t-u} \phi(\phi, \cdot, \infty)+\Theta_{t-u} \phi\left(\sigma^{\circ}, \infty, *\right)-\Theta_{t-u} \phi\left(\Phi^{\prime}, \cdot, *\right)\right] K \rho_{\bar{S}_{u}^{K}}^{K}(\cdot, *) \mathbf{1}_{\odot}, Z_{u}^{K}\right) . \mathbf{1}_{\sigma^{\circ}}, \bar{S}_{u}\right)_{*} d u \\
& -\int_{0}^{t}\left(\left(\left[\Theta_{t-u} \phi(\phi, \cdot, \infty)+\Theta_{t-u} \phi\left(\sigma^{*}, \infty, *\right)-\Theta_{t-u} \phi\left(\Phi^{\prime}, \cdot, *\right)\right] K \rho_{\bar{S}_{u}^{K}}^{K}(\cdot, *) \mathbf{1}_{q}, \bar{S}_{u}\right) . \mathbf{1}_{\sigma^{\prime}}, Z_{u}^{K}\right)_{*} d u \\
& +\int_{0}^{t}\left(\Theta_{t-u} \phi, d \tilde{M}_{u}^{K}\right)
\end{aligned}
$$

where $\tilde{M}$ is the measure such that $\left(f, \tilde{M}_{t}^{K}\right)=\tilde{M}_{t}^{f, K}$. This representation helps establishing the next result, which in turn is used in proving tightness of the sequence $Z^{K}$.

Proposition 19. For any $\epsilon>0$, there exists $R>0$ such that for all $K$,

$$
\mathbb{P}\left(\left\|Z_{t}^{K}\right\|_{W^{-2}}>R\right) \leqslant \epsilon .
$$


Proof. Let $N>\sup _{t \in \mathbb{T}} \bar{X}_{t}^{\infty}$, where $\bar{X}_{t}^{\infty}=\left(\mathbf{1}_{\varphi}+\mathbf{1}_{\sigma^{\circ}}+2 \mathbf{1}_{\varnothing}, \bar{S}_{t}\right)$. Define stopping time $\tau_{N}^{K}=\inf \left\{t \in \mathbb{T}: \bar{X}_{t}^{K}>N\right\}$. Then, we have

$$
\begin{aligned}
\mathbb{P}\left(\left\|Z_{t}^{K}\right\|_{W^{-2}}>R\right) & =\mathbb{P}\left(\left\|Z_{t}^{K}\right\|_{W^{-2}}>R, \tau_{N}^{K}>T\right)+\mathbb{P}\left(\left\|Z_{t}^{K}\right\|_{W^{-2}}>R, \tau_{N}^{K} \leqslant T\right) \\
& \leqslant \frac{1}{R} \mathbb{E}\left[\left\|Z_{t}^{K}\right\|_{W^{-2}} \mathbf{1}_{\tau_{N}^{K}>T}\right]+\mathbb{P}\left(\tau_{N}^{K} \leqslant T\right) .
\end{aligned}
$$

Observe that

$$
\mathbb{P}\left(\tau_{N}^{K} \leqslant T\right)=\mathbb{P}\left(\sup _{t \leqslant T} \bar{X}_{t}^{K}>N\right) \leqslant \frac{1}{N} \mathbb{E}\left[\sup _{t \leqslant T} \bar{X}_{t}^{K}\right]
$$

thus, by (37), for any $\epsilon$, there exists $N$ such that $\mathbb{P}\left(\tau_{N}^{K} \leqslant T\right) \leqslant \epsilon / 2$ for all $K$.

For the first term in (41), we proceed from representation in (23). For $\phi \in W^{2}$, as $\left\|\Theta_{r} \phi\right\|_{W^{2}} \leqslant c\|\phi\|_{W^{2}}$ for $r \in \mathbb{T}$, we have the following:

- $\left|\left(\Theta_{t} \phi, Z_{0}^{K}\right)\right| \leqslant\left\|\Theta_{t} \phi\right\|_{W^{2}}\left\|Z_{0}^{K}\right\|_{W^{-2}} \leqslant c|| \phi\left\|_{W^{2}}\right\| Z_{0}^{K} \|_{W^{-2}}$,

- $\sqrt{K}\left|\left(\Theta_{t-u} \phi\left(q_{\bar{S}_{u}^{K}}^{K}-q_{\bar{S}_{u}}^{\infty}\right), \bar{S}_{u}\right)\right| \leqslant\left\|\Theta_{t} \phi\right\|\left\|_{\infty}\right\| \sqrt{K}\left(q_{\bar{S}_{u}^{K}}^{K}-q_{\bar{S}_{u}}^{\infty}\right)\left\|_{\infty}\left(1, \bar{S}_{u}\right) \leqslant c\right\| \phi \|_{W^{2}}(1+$ $\left.\left\|Z_{u}^{K}\right\|_{W^{-4}}\right) \leqslant c^{\prime}\|\phi\|_{W^{2}}\left(1+\left\|Z_{u}^{K}\right\|_{W^{-2}}\right)$,

- $\left|\left(\Theta_{t-u} \phi q_{\bar{S}_{u}^{K}}^{K}, Z_{u}^{K}\right)\right| \leqslant\left\|\Theta_{t-u} \phi\right\|_{W^{2}}\left\|q_{\bar{S}_{u}^{K}}^{K}\right\|_{C^{2}}\left\|Z_{u}^{K}\right\|_{W^{-2}} \leqslant c\|\phi\|_{W^{2}}\left\|Z_{u}^{K}\right\|_{W^{-2}}$

- $\sqrt{K}\left|\left(\left(\left[\Theta_{t-u} \phi(q, \cdot, \infty)+\Theta_{t-u} \phi\left(\sigma^{*}, \infty, *\right)-\Theta_{t-u} \phi(\Phi, \cdot, *)\right]\left(K \rho_{\bar{S}_{u}^{K}}^{K}(\cdot, *)-\rho_{\bar{S}_{u}}^{\infty}(\cdot, *)\right) \mathbf{1}_{\varphi}, \bar{S}_{u}\right) . \mathbf{1}_{\sigma^{\prime}}, \bar{S}_{u}\right)_{*}\right| \leqslant$ $c|| \phi\left\|_{W^{2}}\left(1+\left\|Z_{u}^{K}\right\|_{W^{-4}}\right)\left(1, \bar{S}_{u}\right)^{2} \leqslant c^{\prime}\right\| \phi \|_{W^{2}}\left(1+\left\|Z_{u}^{K}\right\|_{W^{-2}}\right) \mid$,

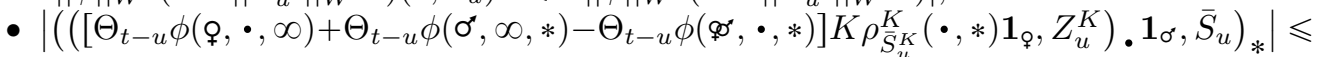
$c|| \Theta_{t} \phi\left\|_{W^{2}}\right\| K \rho_{\bar{S}_{u}^{K}}^{K}\left\|_{C^{2}}\right\| Z_{u}^{K}\left\|_{W^{-2}}\left(1, \bar{S}_{u}\right) \leqslant c^{\prime}\right\| \phi\left\|_{W^{2}}\right\| Z_{u}^{K} \|_{W^{-2}}$,

- $\left|\left(\left(\left[\Theta_{t-u} \phi(\propto, \cdot, \infty)+\Theta_{t-u} \phi\left(\sigma^{\prime \prime}, \infty, *\right)-\Theta_{t-u} \phi\left(\varsigma^{\prime \prime}, \cdot, *\right)\right] K \rho_{\bar{S}_{u}^{K}}^{K}(\cdot, *) \mathbf{1}_{\varphi}, \bar{S}_{u}^{K}\right) . \mathbf{1}_{\sigma^{*}}, Z_{u}^{K}\right)_{*}\right|=$ $\left|\left(\left(\left[\Theta_{t-u} \phi(q, \cdot, \infty)+\Theta_{t-u} \phi\left(\sigma^{\prime \prime}, \infty, *\right)-\Theta_{t-u} \phi(థ, \cdot, *)\right] K \rho_{\bar{S}_{u}^{K}}^{K}(\cdot, *) \mathbf{1}_{\sigma^{*}}, Z_{u}^{K}\right)_{*} \mathbf{1}_{\wp}, \bar{S}_{u}^{K}\right).\right| \leqslant$ $c\|\phi\|_{W^{2}}\left\|Z_{u}^{K}\right\|_{W^{-2}}\left(1, \bar{S}_{u}^{K}\right)$,

- $\int_{0}^{t}\left(\Theta_{t-u} \phi, d \tilde{M}_{u}^{K}\right) \leqslant\|\phi\|_{W^{2}}\left\|\int_{0}^{t} \Theta_{t-u}^{*} d \tilde{M}_{u}^{K}\right\|_{W^{-2}}$,

where we write $\int_{0}^{t} \Theta_{t-u}^{*} d \tilde{M}_{u}^{K}$ for the operator such that $\left(f, \int_{0}^{t} \Theta_{t-u}^{*} d \tilde{M}_{u}^{K}\right)=\int_{0}^{t}\left(\Theta_{t-u} f, d \tilde{M}_{u}^{K}\right)$.

Therefore,

$$
\left\|Z_{t}^{K}\right\|_{W^{-2}} \leqslant c\left(\left\|Z_{0}^{K}\right\|_{W^{-2}}+\int_{0}^{t}\left(1+\left\|Z_{u}^{K}\right\|_{W^{-2}}+\left\|Z_{u}^{K}\right\|_{W^{-2}}\left(1, \bar{S}_{u}^{K}\right)\right) d u\right)+\left\|\int_{0}^{t} \Theta_{t-u}^{*} d \tilde{M}_{u}^{K}\right\|_{W^{-2}} .
$$

Multiply this by $\mathbf{1}_{\tau_{N}^{K}>T}$ and take expectation, we then obtain

$$
\begin{aligned}
& \mathbb{E}\left[\left\|Z_{t}^{K}\right\|_{W^{-2}} \mathbf{1}_{\tau_{N}^{K}>T}\right] \leqslant c_{1}\left(\left\|Z_{0}^{K}\right\|_{W^{-2}} \mathbb{E}\left[\mathbf{1}_{\tau_{N}^{K}>T}\right]\right. \\
&\left.+\int_{0}^{t} \mathbb{E}\left[\left(1+\left\|Z_{u}^{K}\right\|_{W^{-2}}+\left\|Z_{u}^{K}\right\|_{W^{-2}}\left(1, \bar{S}_{u}^{K}\right)\right) \mathbf{1}_{\tau_{N}^{K}>T}\right] d u\right) \\
&+\mathbb{E}\left[\left\|\int_{0}^{t} \Theta_{t-u}^{*} d \tilde{M}_{u}^{K}\right\|_{W^{-2}} \mathbf{1}_{\tau_{N}^{K}>T}\right] \\
& \leqslant c_{2}\left(\left\|Z_{0}^{K}\right\|_{W^{-2}}+t+\int_{0}^{t} \mathbb{E}\left[\left(\left\|Z_{u}^{K}\right\|_{W^{-2}}+\left\|Z_{u}^{K}\right\|_{W^{-2}} N\right) \mathbf{1}_{\tau_{N}^{K}>T}\right] d u\right) \\
&+\mathbb{E}\left[\left\|\int_{0}^{t} \Theta_{t-u}^{*} d \tilde{M}_{u}^{K}\right\|_{W^{-2}}\right]
\end{aligned}
$$

as $\left(1, \bar{S}_{u}^{K}\right) \leqslant \bar{X}_{u}^{K}$ which is less than $N$ on the set $\left\{\tau_{N}^{K}>T\right\}$. To deal with the martingale term, let $\left(p_{l}\right)_{l \geqslant 1}$ denote a complete orthonormal basis of $W^{2}$. Then, for 
$r \leqslant t$,

$$
\begin{aligned}
& \mathbb{E}\left[\left\|\int_{0}^{r} \Theta_{t-u}^{*} d \tilde{M}_{u}^{K}\right\|_{W^{-2}}^{2}\right]=\mathbb{E}\left[\sum_{l \geqslant 1}\left(\int_{0}^{r}\left(\Theta_{t-u} p_{l}, d \tilde{M}_{u}^{K}\right)\right)^{2}\right] \\
& \quad \leqslant \sum_{l \geqslant 1} \mathbb{E}\left[\left\langle\int_{0}^{\cdot}\left(\Theta_{t-u} p_{l}, d \tilde{M}_{u}^{K}\right)\right\rangle_{r}\right] \leqslant \mathbb{E}\left[c \int_{0}^{r}\left(\left(1, \bar{S}_{u}^{K}\right)+\left(1, \bar{S}_{u}^{K}\right)^{2}\right) d u\right] \leqslant c_{T},
\end{aligned}
$$

where the third line is due to $\left|\sum_{l \geqslant 1} p_{l}\left(s_{1}\right) p_{l}\left(s_{2}\right)\right| \leqslant c$ for any $s_{1}, s_{2} \in W^{2}$ and the results in Section B.2. Take $r=t, \mathbb{E}\left[\left\|\int_{0}^{t} \Theta_{t-u}^{*} d \tilde{M}_{u}^{K}\right\|_{W^{-2}}\right]^{2} \leqslant \mathbb{E}\left[\left\|\int_{0}^{t} \Theta_{t-u}^{*} d \tilde{M}_{u}^{K}\right\|_{W^{-2}}^{2}\right] \leqslant$ $c_{T}$. Hence,

$\mathbb{E}\left[\left\|Z_{t}^{K}\right\|_{W^{-2}} \mathbf{1}_{\tau_{N}^{K}>T}\right] \leqslant c_{3}\left(\left\|Z_{0}^{K}\right\|_{W^{-2}}+T+(1+N) \int_{0}^{t} \mathbb{E}\left[\left\|Z_{u}^{K}\right\|_{W^{-2}} \mathbf{1}_{\tau_{N}^{K}>T}\right] d u+1\right)$

and by Gronwall's inequality,

$$
\mathbb{E}\left[\left\|Z_{t}^{K}\right\|_{W^{-2}} \mathbf{1}_{\tau_{N}^{K}>T}\right] \leqslant c_{3}\left(\left\|Z_{0}^{K}\right\|_{W^{-2}}+T+1\right) e^{c_{3}(1+N) T} \leqslant c_{T}(N) .
$$

Thus, for any $\epsilon$ and $N$, there exists $R$ such that $\frac{1}{R} \mathbb{E}\left[\left\|Z_{t}^{K}\right\|_{W^{-2}} \mathbf{1}_{\tau_{N}^{K}>T}\right] \leqslant \epsilon / 2$ for all $K$.

The assertion then follows.

Proposition 20. Let $N>\sup _{t \in \mathbb{T}} \bar{X}_{t}^{\infty}$, where $\bar{X}_{t}^{\infty}=\left(\mathbf{1}_{\propto}+\mathbf{1}_{\sigma^{\circ}}+2 \mathbf{1}_{\phi}, \bar{S}_{t}\right)$. Define stopping time $\tau_{N}^{K}=\inf \left\{t \in \mathbb{T}: \bar{X}_{t}^{K}>N\right\}$. Then,

$$
\mathbb{E}\left[\sup _{t \in \mathbb{T}}\left\|Z_{t}^{K}\right\|_{W^{-3}} \mathbf{1}_{\tau_{N}^{K}>T}\right] \leqslant c_{T}(N) .
$$

Proof. Let $f \in W^{3}$. From (12), take absolute value and bound each term on the right hand side, similar as in Proposition 19. With the inclusions of the spaces, $W^{-2} \hookrightarrow W^{-3} \hookrightarrow W^{-4}$, and the boundedness of the model parameters, we have

$$
\begin{aligned}
& \left|\left(f, Z_{t}^{K}\right)\right| \leqslant\|f\|_{W^{3}}\left(\left\|Z_{0}^{K}\right\|_{W^{-3}}\right. \\
& \left.\quad+c_{1} \int_{0}^{t}\left(1+\left\|Z_{u}^{K}\right\|_{W^{-2}}+\left\|Z_{u}^{K}\right\|_{W^{-2}}\left(1, \bar{S}_{u}^{K}\right)\right) d u+\left\|\tilde{M}_{t}^{K}\right\|_{W^{-3}}\right) .
\end{aligned}
$$

Thus,

$$
\left\|Z_{t}^{K}\right\|_{W^{-3}} \leqslant\left\|Z_{0}^{K}\right\|_{W^{-3}}+c_{1} \int_{0}^{t}\left(1+\left\|Z_{u}^{K}\right\|_{W^{-2}}\left(1+\left(1, \bar{S}_{u}^{K}\right)\right)\right) d u+\left\|\tilde{M}_{t}^{K}\right\|_{W^{-3}}
$$

and

$$
\begin{aligned}
\mathbb{E}\left[\sup _{t \in \mathbb{T}}\left\|Z_{t}^{K}\right\|_{W-3} \mathbf{1}_{\tau_{N}^{K}>T}\right] \leqslant\left\|Z_{0}^{K}\right\|_{W^{-3}} \\
\quad+c_{1}\left(T+(1+N) \int_{0}^{T} \mathbb{E}\left[\left\|Z_{u}^{K}\right\|_{W^{-2}} \mathbf{1}_{\tau_{N}^{K}>T}\right] d u\right)+\mathbb{E}\left[\sup _{t \in \mathbb{T}}\left\|\tilde{M}_{t}^{K}\right\|_{W^{-3}}\right] .
\end{aligned}
$$

Now, let $\left(p_{l}\right)_{l \geqslant 1}$ denote a complete orthonormal basis of $W^{3}$. Then,

$$
\begin{aligned}
\mathbb{E}\left[\sup _{t \in \mathbb{T}}\left\|\tilde{M}_{t}^{K}\right\|_{W^{-3}}\right] & =\mathbb{E}\left[\sup _{t \in \mathbb{T}} \sum_{l \geqslant 1}\left(\tilde{M}_{t}^{p_{l}, K}\right)^{2}\right] \leqslant \sum_{l \geqslant 1} \mathbb{E}\left[\sup _{t \in \mathbb{T}}\left(\tilde{M}_{t}^{p_{l}, K}\right)^{2}\right] \\
& \leqslant 4 \sum_{l \geqslant 1} \mathbb{E}\left[\left\langle\tilde{M}^{p_{l}, K}\right\rangle_{T}\right] \leqslant c \int_{0}^{T} \mathbb{E}\left[\left(1, \bar{S}_{u}^{K}\right)+\left(1, \bar{S}_{u}^{K}\right)^{2}\right] d u \leqslant c_{T} .
\end{aligned}
$$


From the proof of Proposition [19, we also have $\mathbb{E}\left[\left\|Z_{u}^{K}\right\|_{W^{-2}} \mathbf{1}_{\tau_{N}^{K}>T}\right] \leqslant c_{T}(N)$. The assertion thus follows.

Proposition 21. The sequence $Z^{K}$ is tight in $\mathbb{D}\left(\mathbb{T}, W^{-4}\right)$.

Proof. In a similar way as in Proposition 14, (T1) follows from Proposition 19.

For (T2a), observe that

$\mathbb{P}\left(\left\|\tilde{V}_{\tau^{K}+\zeta \wedge T}^{K}-\tilde{V}_{\tau^{K}}^{K}\right\|_{W^{-4}}>\epsilon_{1}\right) \leqslant \mathbb{P}\left(\left\|\tilde{V}_{\tau^{K}+\zeta \wedge T}^{K}-\tilde{V}_{\tau^{K}}^{K}\right\|_{W^{-4}}>\epsilon_{1}, \tau_{N}^{K}>T\right)+\mathbb{P}\left(\tau_{N}^{K} \leqslant T\right)$.

As shown in the proof of Proposition 19, for any $\epsilon_{2}$, there exists $N$ such that $\mathbb{P}\left(\tau_{N}^{K} \leqslant T\right)<\epsilon_{2} / 2$. Using same technique as before, we can also show that

$$
\left\|\tilde{V}_{\tau^{K}+\zeta \wedge T}^{K}-\tilde{V}_{\tau^{K}}^{K}\right\|_{W^{-4}} \leqslant c \int_{\tau^{K}}^{\tau^{K}+\zeta \wedge T}\left(1+\left\|Z_{u}^{K}\right\|_{W^{-3}}\left(1+\left(1, \bar{S}_{u}^{K}\right)\right)\right) d u
$$

and thus

$$
\begin{aligned}
\mathbb{E}\left[\left\|\tilde{V}_{\tau^{K}+\zeta \wedge T}^{K}-\tilde{V}_{\tau^{K}}^{K}\right\|_{W^{-4}} \mathbf{1}_{\tau_{N}^{K}>T}\right] & \leqslant c\left(\zeta+\mathbb{E}\left[\int_{\tau^{K}}^{\tau^{K}+\zeta \wedge T}\left\|Z_{u}^{K}\right\|_{W^{-3}}\left(1+\left(1, \bar{S}_{u}^{K}\right)\right) d u \mathbf{1}_{\tau_{N}^{K}>T}\right]\right) \\
& \leqslant c\left(\zeta+(1+N) \mathbb{E}\left[\int_{0}^{\zeta}\left\|Z_{\tau^{K}+u \wedge T}^{K}\right\|_{W^{-3}} d u \mathbf{1}_{\tau_{N}^{K}>T}\right]\right) \\
& \leqslant c\left(\zeta+(1+N) \int_{0}^{\zeta} \mathbb{E}\left[\sup _{u \leqslant T}\left\|Z_{u}^{K}\right\|_{W^{-3}} \mathbf{1}_{\tau_{N}^{K}>T}\right] d u\right) \\
& \leqslant c_{T}(N) \delta
\end{aligned}
$$

where the last inequality follows from Proposition 20. Therefore,

$$
\begin{aligned}
\mathbb{P}\left(\left\|\tilde{V}_{\tau^{K}+\zeta \wedge T}^{K}-\tilde{V}_{\tau^{K}}^{K}\right\|_{W^{-4}}>\epsilon_{1}, \tau_{N}^{K}>T\right) & \leqslant \frac{1}{\epsilon_{1}} \mathbb{E}\left[\left\|\tilde{V}_{\tau^{K}+\zeta \wedge T}^{K}-\tilde{V}_{\tau^{K}}^{K}\right\|_{W^{-4}} \mathbf{1}_{\tau_{N}^{K}>T}\right] \\
& \leqslant \frac{c_{T}(N) \delta}{\epsilon_{1}},
\end{aligned}
$$

and for any $\epsilon_{1}, \epsilon_{2}$ and $N$, there exists $\delta$ such that the probabilty does not exceed $\epsilon_{2} / 2$.

For (T2b), let $\left(p_{l}\right)_{l \geqslant 1}$ denote a complete orthonormal basis of $W^{4}$. Note that $\left\|\tilde{M}_{t}^{K}\right\|_{W^{-4}}^{2}=\sum_{l \geqslant 1}\left(\tilde{M}_{t}^{p_{l}, K}\right)^{2}$ and $\left\langle\left\langle\tilde{M}^{K}\right\rangle\right\rangle_{t}=\sum_{l \geqslant 1}\left\langle\tilde{M}^{p_{l}, K}\right\rangle_{t}$. So,

$$
\begin{aligned}
\mathbb{E}\left[\left|\left\langle\left\langle\tilde{M}^{K}\right\rangle\right\rangle_{\tau^{K}+\zeta \wedge T}-\left\langle\left\langle\tilde{M}^{K}\right\rangle\right\rangle_{\tau^{K}}\right|\right] & =\mathbb{E}\left[\left|\sum_{l \geqslant 1}\left\langle\tilde{M}^{p_{l}, K}\right\rangle_{\tau^{K}+\zeta \wedge T}-\sum_{l \geqslant 1}\left\langle\tilde{M}^{p_{l}, K}\right\rangle_{\tau^{K}}\right|\right] \\
& \leqslant \mathbb{E}\left[c \int_{\tau^{K}}^{\tau^{K}+\zeta \wedge T}\left(\left(1, \bar{S}_{u}^{K}\right)+\left(1, \bar{S}_{u}^{K}\right)^{2}\right) d u\right] \\
& \leqslant c \delta\left(\mathbb{E}\left[\sup _{u \leqslant T}\left(1, \bar{S}_{u}^{K}\right)\right]+\mathbb{E}\left[\sup _{u \leqslant T}\left(1, \bar{S}_{u}^{K}\right)^{2}\right]\right) \\
& \leqslant c_{T} \delta .
\end{aligned}
$$

Proposition 22. $Z^{K}$ and $\tilde{M}^{K}$ are $C$-tight, that is, all limit points of $Z^{K}$ and $\tilde{M}^{K}$ are in $\mathbb{C}\left(\mathbb{T}, W^{-4}\right)$. 
Proof. Note that $Z^{K}$ jumps when $S^{K}$ jumps, which jumps when there is an event associated with birth, death or marriage. Let $f \in W^{4}$. Then, assuming that the number of offspring is bounded by $\Xi$ which has finite mean and variance,

$$
\begin{aligned}
& \left|\left(f, \Delta Z_{t}^{K}\right)\right|=\frac{1}{\sqrt{K}}\left|\left(f, S_{t}^{K}-S_{t-}^{K}\right)\right| \\
& \quad \leqslant \frac{1}{\sqrt{K}}\left(|f(1,0, \infty)| \Xi+|f(2, \infty, 0)| \Xi+\sup _{v}|f(1, v, \infty)|+\sup _{w}|f(2, \infty, w)|+\sup _{v, w}|f(3, v, w)|\right) \\
& \quad \leqslant \frac{1}{\sqrt{K}} c|| f \|_{\infty}(1+\Xi)
\end{aligned}
$$

and

$$
\mathbb{E}\left[\sup _{t \leqslant u}\left\|\Delta Z_{t}^{K}\right\|_{W^{-4}}\right] \leqslant \frac{c}{\sqrt{K}}(1+\mathbb{E}[\Xi]) .
$$

It follows that, for any $u$ and $\epsilon>0$,

$$
\mathbb{P}\left(\sup _{t \leqslant u}\left\|\Delta Z_{t}^{K}\right\|_{W^{-4}}>\epsilon\right) \leqslant \frac{1}{\epsilon} \mathbb{E}\left[\sup _{t \leqslant u}\left\|\Delta Z_{t}^{K}\right\|_{W^{-4}}\right] \leqslant \frac{c}{\epsilon \sqrt{K}}(1+\mathbb{E}[\Xi]),
$$

which converges to zero as $K$ tends to infinity. This, together with the tightness of $Z^{K}$, shows that the sequence is C-tight (see e.g. [14, Proposition VI.3.26]).

Similarly, $\tilde{M}^{K}$ is C-tight, since $\tilde{M}^{K}$ has the same jumps as $Z^{K}$.

Proposition 23. $\tilde{M}^{K}$ converges in $\mathbb{D}\left(\mathbb{T}, W^{-4}\right)$ to $\tilde{M}^{\infty}$, defined such that for each $f \in W^{4},\left(f, \tilde{M}^{\infty}\right)=\tilde{M}^{f, \infty}$ is a martingale with predictable quadratic variation (33).

Proof. See proof of Proposition 16

Finally, Theorem 4 follows.

of Theorem 4. Every limit point $z$ of $Z^{K}$ satisfies, for $\phi \in W^{4}$,

$\left(\phi, z_{t}\right)=\left(\Theta_{t} \phi, z_{0}\right)$

$$
\begin{aligned}
& -\int_{0}^{t}\left(\Theta_{t-u} \phi \partial_{S} h_{\bar{S}_{u}, \infty}\left(z_{u}\right) \mathbf{1}_{\varphi}+\Theta_{t-u} \phi \partial_{S} h_{\bar{S}_{u}^{\sigma, \infty}}^{\sigma_{u}}\left(z_{u}\right) \mathbf{1}_{\sigma^{\sigma}}, \bar{S}_{u}\right) d u-\int_{0}^{t}\left(\Theta_{t-u} \phi h_{\bar{S}_{u}, \infty}^{\infty} \mathbf{1}_{\varphi}+\Theta_{t-u} \phi h_{\bar{S}_{u}, \infty}^{\sigma,} \mathbf{1}_{\sigma}, z_{u}\right) d u \\
& +\int_{0}^{t}\left(\left[\left(\Theta_{t-u} \phi\left(\sigma^{\circ}, \infty, \cdot\right)-\Theta_{t-u} \phi\left(\phi^{\circ}, \cdot, \cdot\right)\right) \partial_{S} h_{\bar{S}_{u}, \infty}\left(z_{u}\right)+\left(\Theta_{t-u} \phi(q, \cdot, \infty)-\Theta_{t-u} \phi\left(\phi^{\prime}, \cdot, \cdot\right)\right) \partial_{S} h_{\bar{S}_{u}}^{\sigma, \infty}\left(z_{u}\right)\right] \mathbf{1}_{\mathbf{1}^{\prime}}, \bar{S}_{u}\right) d u
\end{aligned}
$$

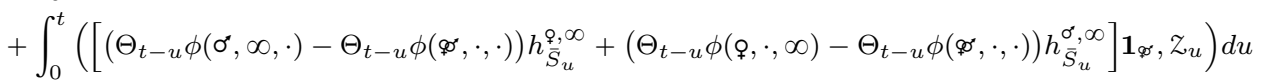

$$
\begin{aligned}
& +\int_{0}^{t}\left(\left(\Theta_{t-u} \phi(\wp, \cdot, \infty)+\Theta_{t-u} \phi\left(\sigma^{\infty}, \infty, \cdot\right)-\Theta_{t-u} \phi\left(\Phi^{\infty}, \cdot, \cdot\right)\right) \partial_{S} h_{\bar{S}_{u}, \infty}^{\infty}\left(z_{u}\right) \mathbf{1}_{\infty}, \bar{S}_{u}\right) d u \\
& +\int_{0}^{t}\left(\left(\Theta_{t-u} \phi(q, \cdot, \infty)+\Theta_{t-u} \phi\left(\sigma^{\prime \prime}, \infty, \cdot\right)-\Theta_{t-u} \phi(\Phi, \cdot, \cdot)\right) h_{\bar{S}_{u}, \infty}^{\infty} \mathbf{1}_{\phi}, z_{u}\right) d u \\
& +\int_{0}^{t}\left(\left[\Theta_{t-u} \phi(\phi, 0, \infty) \partial_{S}\left(b_{\bar{S}_{u}}^{\infty} m_{\bar{S}_{u}}^{\varrho}\right)\left(z_{u}\right)+\Theta_{t-u} \phi\left(\sigma^{\infty}, \infty, 0\right) \partial_{S}\left(b_{\bar{S}_{u}}^{\infty} m_{\bar{S}_{u}}^{\sigma}\right)\left(z_{u}\right)\right]\left(\mathbf{1}_{\varnothing}+\mathbf{1}_{\wp}\right), \bar{S}_{u}\right) d u \\
& +\int_{0}^{t}\left(\left[\Theta_{t-u} \phi(\phi, 0, \infty) m_{\bar{S}_{u}, \infty}+\Theta_{t-u} \phi\left(\sigma^{\circ}, \infty, 0\right) m_{\bar{S}_{u}, \infty}^{\sigma}\right] b_{\bar{S}_{u}}^{\infty}\left(\mathbf{1}_{\phi^{\prime}}+\mathbf{1}_{\varphi}\right), z_{u}\right) d u \\
& -\int_{0}^{t}\left(\left(\left[\Theta_{t-u} \phi(q, \cdot, \infty)+\Theta_{t-u} \phi\left(\sigma^{*}, \infty, *\right)-\Theta_{t-u} \phi(\Phi, \cdot, *)\right] \partial_{S} \rho_{\bar{S}_{u}}^{\infty}\left(z_{u}\right)(\cdot, *) \mathbf{1}_{q}, \bar{S}_{u}\right) ._{\boldsymbol{1}^{\prime}}, \bar{S}_{u}\right)_{*} d u \\
& -\int_{0}^{t}\left(\left(\left[\Theta_{t-u} \phi(\phi, \cdot, \infty)+\Theta_{t-u} \phi\left(\sigma^{*}, \infty, *\right)-\Theta_{t-u} \phi\left(\Phi^{\circ}, \cdot, *\right)\right] \rho_{\bar{S}_{u}}^{\infty}(\cdot, *) \mathbf{1}_{q}, z_{u}\right) . \mathbf{1}_{\sigma^{*}}, \bar{S}_{u}\right)_{*} d u \\
& -\int_{0}^{t}\left(\left(\left[\Theta_{t-u} \phi(\phi, \cdot, \infty)+\Theta_{t-u} \phi\left(\sigma^{*}, \infty, *\right)-\Theta_{t-u} \phi(\Phi, \cdot, *)\right] \rho_{\bar{S}_{u}}^{\infty}(\cdot, *) \mathbf{1}_{\varphi}, \bar{S}_{u}\right) . \mathbf{1}_{\sigma^{\prime}}, z_{u}\right)_{*} d u \\
& +\int_{0}^{t}\left(\Theta_{t-u} \phi, d \tilde{M}_{u}^{\infty}\right) \text {. }
\end{aligned}
$$


Showing the uniqueness of the solution to (42) and that (42) is equivalent to (13) completes the proof.

\section{REFERENCES}

[1] R.A. Adams, J.J.F. Fournier (2003). Sobolev Spaces, 2nd ed. Elsevier.

[2] S. Asmusses (1980). On some two-sex population models. The Annals of Probability 8(4), $727-744$.

[3] A.-M. Borde-Boussion (1990). Stochastic demographic models: Age of a population. Stochastic Processes and their Application 35, 279-291.

[4] D.J. Daley (1968). Extinction conditions for certain bisexual Galton-Watson branching processes. Z. Wahrscheinlichkeitstheorie verw. Geb. 9, 315-322.

[5] C. Dellacherie, P.A. Meyer (1978). Probabilities and Potential. North-Holland.

[6] S. Engen, R. Lande, B-E. Sæther (2003). Demographic stochasticity and Allee effects in populations with two sexes. Ecology 84(9), 2378-2386.

[7] J.Y. Fan, K. Hamza, P. Jagers, F.C. Klebaner (2019). Convergence of the age structure of general schemes of population processes. Accepted in Bernoulli.

[8] A.G. Fredrickson (1971). A mathematical theory of age structure in sexual populations: random mating and monogamous marriage models. Mathematical Biosciences 10, 117-143.

[9] J.H. Gillespie (2004). Population Genetics: A Concise Guide, 2nd ed. Johns Hopkins University Press.

[10] K. Hamza, P. Jagers, F.C. Klebaner (2016). On the establishment, persistence and inevitable extinction of populations. J. Math. Biology 72, 797-820. DOI: 10.1007/s00285-015-0903-2.

[11] K. Hamza, P. Jagers, F.C. Klebaner (2013). The age structure of population-dependent general branching processes in environments with a high carrying capacity. Proceedings of the Steklov Institute of Mathematics 282, 90-105.

[12] T.E. Harris (1963). The Theory of Branching Processes. Springer.

[13] O. Hyrien, M. Mayer-Pröschel, M. Noble, A. Yakovlev (2005). A stochastic model to analyze clonal data on multi-type cell populations. Biometrics 61, 199-207.

[14] J. Jacod, A.N. Shiryaev (2003). Limit Theorems for Stochastic Processes, 2nd ed. Springer.

[15] P. Jagers (1974) Aspects of random measures and point processes. In:P. Ney and S. Port (Eds). Advances in Probability Theory 3. Marcel Dekker, New York.

[16] P. Jagers (1975). Branching Processes with Biological Applications. John Wiley \& Sons.

[17] P. Jagers (1989). General branching processes as Markov fields. Stoch. Proc. Appl. 32, 183212.

[18] P. Jagers, F.C. Klebaner (2000). Population-size-dependent and age-dependent branching processes. Stochastic Processes and Their Applications 87, 235-254.

[19] P. Jagers, F.C. Klebaner (2011). Population-size-dependent, age-structured branching processes linger around their carrying capacity. Journal of Applied Probability 48, 249-260.

[20] P. Jagers, F.C. Klebaner (2016). From size to age and type structure dependent branching: a first step to sexual reproduction in general population processes. Branching Processes and their Applications (I. M. del Puerto et al., eds.), Springer Lecture Notes in Statistics, 137-148.

[21] A. Jakubowski (1986). On the Skorokhod topology. Ann. Inst. Henri Poincaré, Probab. Stat. 22(3), 263-285.

[22] O. Kallenberg (2017) Random Measures, Theory and Applications. Springer Nature.

[23] F.C. Klebaner (1984). Geometric rate of growth in population size dependent branching processes. J. Appl. Prob. 21, 40-49.

[24] F.C. Klebaner (1989). Geometric growth in near-supercritical population size dependent multitype Galton-Watson processes. Ann. Prob. 17(4), 1466-1477.

[25] F.C. Klebaner (1994). Asymptotic behaviour of Markov population processes with asymptotically linear rate of change. J. Appl. Prob. 31, 614-625.

[26] S. Meleard (1998). Convergence of the fluctuations for interacting diffusions with jumps associated with Boltzmann equations. Stochastics and Stochastic Reports 63, 195-225.

[27] M. Metivier (1987). Weak convergence of measure valued processes using Sobolev-imbedding techniques. Lecture Notes in Mathematics 1236, 172-183. Springer, Berlin.

[28] M. Molina, M. Mota, A, Ramos (2006) On $L^{\alpha}$-convergence $(1 \leqslant \alpha \leqslant 2)$ for a bisexual branching process with population-size dependent mating. Bernoulli 12(3), 457-468. 
FAN ET AL.

[29] M. Molina, N. Yanev (2003). Continuous time bisexual branching processes. Comptes rendus de l'Académie bulgare des Sciences 56(8), 5-10.

[30] R.E. Nordon, K.-H. Ko, R. Odell, T. Schroeder (2011). Multi-type branching models to describe cell differentiation programs. Journal of Theoretical Biology 277, 7-18.

[31] A.G. Rossberg (2013). Food Webs and Biodiversity: Foundations, Models, Data. John Wiley \& Sons.

School of Mathematical Sciences, Monash University, Clayton, Vic. 3058, AusTRALIA.

E-mail address: jieyen.fan@monash.edu

School of Mathematical Sciences, Monash University, Clayton, Vic. 3058, AusTRALIA.

E-mail address: kais.hamza@monash.edu

Department of Mathematical Sciences, Chalmers University of Technology.

E-mail address: jagers@chalmers.se

School of Mathematical Sciences, Monash University, Clayton, Vic. 3058, AusTRALIA.

E-mail address: fima.klebaner@monash.edu 